\title{
Approaches and methods to assess sedentary behavior in Brazil: a systematic review
}

\author{
Abordagens e métodos para avaliar o comportamento sedentário no Brasil: uma \\ revisão sistemática
}

\begin{abstract}
AUTHOR'S
Luciana Leite Silva Barboza ${ }^{1}$ (D)

Ellen Caroline Mendes da Silva² (iD

Mabliny Thuany ${ }^{3}$ (D)

Raphael Henrique de Oliveira Araujo² (D)

André Oliveira Werneck ${ }^{4}$ (D)

David Nunes Oliveira ${ }^{1}$ (D)

Larissa Gandarela ${ }^{5}$ (D)

Danilo Rodrigues Pereira da Silva ${ }^{5}$ (iD

1 Universidade Federal de Sergipe, Programa de

Pós-graduação em Educação Física, São Cristóvão,

Sergipe, Brasil.

2 Universidade Estadual de Londrina, Programa

de Pós-graduação em Ciências da Saúde, Londrina,

de Pós-gradua

3 Universidade do Porto, Centro de Investigação,

Formação, Inovação e Intervenção em Desporto,

Faculdade de Desporto, Porto, Portugal.

4 Universidade de São Paulo, Faculdade de Saúde

Pública, Núcleo de Pesquisas Epidemiológicas em
Nutrição e Saúde, Departamento de Nutrição, São

Paulo, São Paulo, Brasil.

5 Universidade Federal de Sergipe, Departamento

de Educação Física, São Cristơvão, Sergipe, Brasil.
\end{abstract}

\section{CORRESPONDING}

Luciana Leite Silva Barboza

leite.lu@gmail.com

Av. Marechal Rondon, s/n, Rosa Elze, São

Cristóvão, Sergipe, Brazil.

Postal code: 49100-000.

DOI

10.12820/rbafs.26e0236

\section{(c) BY}

This work is licensed under a Creative Commons Attribution 4.0 International License.

\begin{abstract}
Our aim was to characterize the Brazilian studies that evaluated sedentary behavior, describing the main characteristics of the studies and identifying the used instruments. Using the PRISMA methodology, the search occurred on 25 August 2019 and was updated on 17 October 2020 in the following databases: PUBMED, LILACS, and SCIELO. Studies in English, Spanish, and Portuguese were included. The inclusion criteria were studies performed with a sample of Brazilians, and that used an instrument to evaluate sedentary behavior. In total, 229 articles were selected. The majority of the studies evaluated children and adolescents. Only 33 studies used device-derived measures and there was a great variability in the questionnaires used. Only 83/198 studies presented quality criteria for the instrument used. Most studies considered the screen time in a typical week plus weekend to characterize sedentary behavior. Therefore, sedentary behavior in Brazil has mostly been evaluated by different questionnaires, and few have been appropriately validated. These findings emphasize the importance of standardization and methodological rigor for assessing sedentary behavior in the Brazilian context.
\end{abstract}

Keywords: Healthy lifestyle; Sedentary lifestyle; South America.

\section{RESUMO}

Nosso objetivo foi caracterizar os estudos brasileiros que avaliaram o comportamento sedentário, descrevendo as principais caracteristicas dos estudos e identificando os instrumentos utilizados. Utilizando a metodologia PRISMA, a busca ocorreu em 25 de agosto de 2019 e foi atualizada em 17 de outubro de 2020 nas seguintes bases de dados: PUBMED, LILACS e SCIELO. Estudos em inglês, espanhol e português foram incluidos. Os critérios de inclusão foram estudos realizados com uma amostra de brasileiros e que utilizassem um instrumento para avaliar o comportamento sedentário. No total, foram selecionados 229 artigos. A maioria dos estudos avaliou crianças e adolescentes. Apenas 33 estudos usaram medidas derivadas de dispositivos e houve uma grande variabilidade nos questionários usados. Apenas 83/198 estudos apresentaram critérios de qualidade para o instrumento utilizado. A maioria dos estudos considerou o tempo de tela em uma semana típica mais o fim de semana para caracterizar o comportamento sedentário. Portanto, o comportamento sedentário no Brasil tem sido avaliado em grande parte por diferentes questionários, e poucos foram devidamente validados. Esses achados enfatizam a importância da padronização e do rigor metodológico para avaliação do comportamento sedentário no contexto brasileiro.

Palavras-chave: Estilo de vida saudável; Estilo de vida sedentário; América do Sul.

\section{Introduction}

Sedentary behavior (SB) is characterized as a waking behavior with an energy expenditure of up to 1.5 METs (metabolic equivalent of task), in the sitting, lying, or reclining positions ${ }^{1}$, manifested in different domains of the day (e.g., leisure, work-study, trave- ling, at home), context (e.g., screen time, talking with friends, studying), and patterns (duration of bouts and number of breaks) ${ }^{2}$. Emerging studies also have pointed out $\mathrm{SB}$ as a major public health issue, as the excess of SB is associated with a higher probability of cardiovascular and metabolic diseases and death from all cau- 
$\operatorname{ses}^{3-5}$. Therefore, several studies have been developed to understand the relationship between this behavior and health outcomes ${ }^{6}$, identifying methods capable of reliably measuring these manifestations can help guide more assertive recommendations for populations.

Some countries have developed public health guidelines that include recommendations about $\mathrm{SB}^{7-11}$. These guidelines are generally based on epidemiological studies, which evaluate SB using device-derived (accelerometry and devices that detect postural changes) and subjective methods (questionnaires) ${ }^{12}$. Device-derived methods, with the use of accelerometers and inclinometers, are used more frequently in studies from developed countries. These types of measurements are more accurate than subjective methods ${ }^{13}$, such as questionnaires and diaries $/ \log s^{14}$, as they can perform evaluations according to body acceleration or the postural transitions, besides eliminating the memory bias of the individual ${ }^{15}$. Although self-reports have the advantage of determining the context and type of activity performed ${ }^{13}$, individuals tend to underestimate sedentary behavior with the use of subjective methods, compared to device-derived methods ${ }^{16}$.

On the other hand, middle-income countries, such as Brazil, have differences in context and available resources for research development. It can make it difficult to access and use device-derived methods to measure $\mathrm{SB}$ in research. Besides that, it is not known how the instruments for obtaining information on $\mathrm{SB}$ are being used, for example, whether they present quality indicators, which domain they assess, or what period of time they include when they are applied (e.g., the previous 7 days, habitual behavior, or the previous week). Identifying the most frequently used methods in Brazil could help to disseminate more reliable methods, increasing the standardization and comparability between studies; to show the quality of information available, and to reflect on the need for new methods/ tools for reliably monitoring SB. Thus, the aim of this systematic review was to characterize the Brazilian studies that evaluated SB to verify the frequently used methods, the main characteristics, and whether the studies present indicators of psychometric quality of these methods.

\section{Methods}

The methodology for conducting the study followed the PRISMA guidelines for systematic reviews and meta-analyses ${ }^{17}$ and the protocol was registered with
PROSPERO under the number CRD42020147361.

The search occurred on 25 August 2019 and was updated on 17 October 2020, in the following databases: PUBMED, LILACS, and SCIELO. The search terms used in PUBMED were: (Sedentary OR "sedentary lifestyle" OR "sedentary behavior" OR "sedentary behaviour" OR "screen time" OR screen-view* OR "TV view"” OR "TV watch"” OR "video game" OR "computer use" OR "sitting time" OR sitting OR smartphone OR "mobile phone”) AND (Brazil OR Brasil), with the "humans" filter applied. In the LILACS and SCIELO databases, the terms were ("sedentary behavior" OR "sedentary behaviour" OR "screen time" OR "sitting time" OR "TV viewing" OR "TV watching” OR “computer use" OR video-game OR videogame) AND (Brazil OR Brasil).

Original articles published in English, Spanish, and Portuguese, without time restriction were eligible for inclusion. Additionally, we considered: 1) studies performed with a sample of Brazilians and 2) which used an instrument for the evaluation of SB. We excluded studies that were systematic reviews, meta-analyses, study protocols, or performed with animals or in other areas of knowledge. There were no criteria regarding the type of study (cross-sectional, longitudinal, case studies), age range, or sample size.

The identification and screening steps were carried out using the StArt software (State of the Art through Systematic Review, UFSCAR, version 3.4 beta), by two independent reviewers (Barboza LLS; Silva ECM), who resolved the disagreements in a consensual agreement. After identification and exclusion of duplicate articles, the reviewers evaluated the articles by title and abstract. Potential eligible articles were evaluated posteriorly based on a complete reading of the methodology. Finally, the articles included had the data extracted by the same two reviewers, with the data checked by two other reviewers (Oliveira DN e Gandarela L). The data extracted, organized into an Excel worksheet, were: author, year, region, city-state or only state, sample size, sample characteristics and the cutoff point for SB, for all studies; days of use, minimum hours of use, valid minimum days, epochs and device model, for studies that used device-derived methods; questionnaire used, if show quality indicator, SB indicator, and time reference, for studies that used subjective methods. In this step, among studies that presented data from the same sample, only the oldest study (by the date of publication) was accepted, while the others 
were excluded. In our study, in relation to the subjective methods, studies that explicitly presented in the text or that cited any study with a quality indicator, such as reproducibility or validity of the instrument, were considered to have a positive quality indicator.

\section{Results}

Initially, 2553 articles were found. Of these, 288 were excluded as they were duplicated, and another 1814 because they did not meet the inclusion criteria for the review, so 229 were selected (Figure 1). The first studies were from 1998. The years that presented the highest number of publications were 2017 ( $\mathrm{n}=29), 2018$ ( $\mathrm{n}$ $=31)$, and $2019(n=27)$. The regions of Brazil that presented more studies with samples from their states were the Southeast, South, and Northeast, with, respectively, 80, 70, and 43 publications. Considering all selected studies, the evaluated sample ranged from 12 to 410,684 individuals, with the majority being children and adolescents $(n=146)$, of which 124 were preschoolers or students. The adults or the elderly comprised the sample of the other 83 studies, 15 of which included a special health condition. 31 studies used only device-derived measures; another 2 used both device-derived and subjective measures, and the majority used only questionnaires $(n=196)$. All information extracted from the selected studies is presented in the supplementary material, divided into studies that used device-derived measurement instruments to assess SB (supplementary table 1), studies that used subjective measurement instruments to assess SB in children or adolescents (supplementary table 2) and studies that used subjective measurement instruments to assess SB in adults and the elderly (supplementary Table 3 ).

Among the 33 studies that used device-derived measures, with accelerometers or other motion sensors (Table 1), the first is from 2014, with the majority being published in $2018(\mathrm{n}=9)$ using a sample from the Southeast region $(n=14)$, and being performed in adults or elderly (19). The most widely used device in 25 studies was the ActiGraph accelerometer, models GT3X, GT3X+, GT3X Plus, 7164, GT1M, GT9X, or WGT3X-BT. Most studies followed the protocol for use of seven consecutive days ( $\mathrm{n}=22$ studies), considering at least 10 hours for a valid day $(\mathrm{n}=16)$ and a minimum of four days of use $(n=10)$. The majority adopted 60 -second epochs $(n=11)$ and rated SB below the range of 100 counts per minute or 25 counts per 15 seconds $(n=18)$.

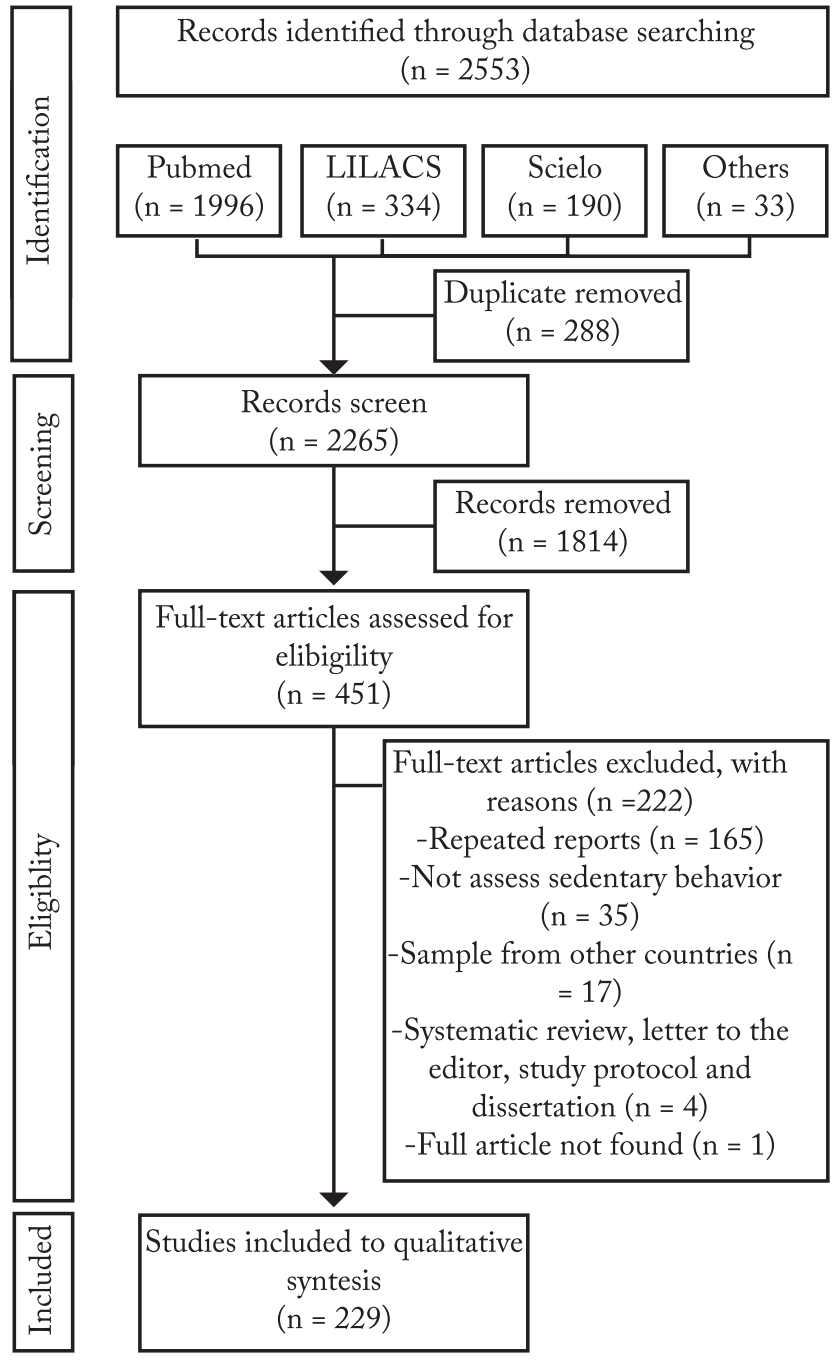

Figure 1 - Flow-chart of search results.

Of the studies that used questionnaires (Table 2), 104 specified the instrument used, and only 83 presented quality indicators, such as validity or reproducibility, indicated in the study itself or previous studies; another 94 studies did not specify the questionnaire and 115 did not present quality indicators of the SB measure. The most frequently used questionnaire, cited in 27 studies, was the International Physical Activity Questionnaire (IPAQ), followed by Global schoolbased student health questionnaire (GSHS) cited in 11 studies, PAQ-C and VIGITEL, cited in 9 studies each; COMPAC appeared in 6 studies each; ASAQ and Baecke Questionnaire, used in 5 studies each. Regarding the contexts of the measure, 96 studies evaluated only screen time (television, computer, video game, and/or other electronic devices) for SB characterization, while 36 others considered only sitting time and 40 only TV watching. 70 studies did not specify the 
Table 1 - Main characteristics of studies that assessed sedentary behavior using device-derived methods, according to the sample.

\begin{tabular}{|c|c|c|}
\hline \multirow[b]{2}{*}{$\begin{array}{l}\text { Characteristics } \\
\text { of the studies }\end{array}$} & \multicolumn{2}{|c|}{ Number of studies } \\
\hline & $\begin{array}{l}\text { Children or } \\
\text { adolescents } \\
\quad(\mathrm{n}=14)\end{array}$ & $\begin{array}{c}\text { Adults or } \\
\text { elderly } \\
(\mathrm{n}=19)\end{array}$ \\
\hline \multicolumn{3}{|l|}{ Year of publication } \\
\hline $2014-2016$ & 4 & 3 \\
\hline $2017-2020$ & 10 & 16 \\
\hline \multicolumn{3}{|l|}{ Region } \\
\hline Brazil or more than one region & 0 & 3 \\
\hline Northeast & 2 & 2 \\
\hline South & 6 & 6 \\
\hline Southeast & 6 & 8 \\
\hline \multicolumn{3}{|l|}{ Sample size } \\
\hline$<100$ & 4 & 10 \\
\hline $100-499$ & 8 & 5 \\
\hline $500-999$ & 2 & 2 \\
\hline $1000-5000$ & 0 & 2 \\
\hline \multicolumn{3}{|l|}{ Days of use } \\
\hline$<7$ days & 5 & 3 \\
\hline 7 days & 8 & 14 \\
\hline$>7$ days & 1 & 1 \\
\hline $\mathrm{N} / \mathrm{I}$ & 0 & 1 \\
\hline \multicolumn{3}{|l|}{ Minimum hours of use } \\
\hline$<10$ hours & 5 & 1 \\
\hline 10 hours & 5 & 11 \\
\hline$>10$ hours & 0 & 3 \\
\hline $\mathrm{N} / \mathrm{I}$ & 4 & 4 \\
\hline \multicolumn{3}{|l|}{ Cut-off points } \\
\hline$<100 \mathrm{cpm}$ & 6 & 12 \\
\hline Others & 7 & 4 \\
\hline $\mathrm{N} / \mathrm{I}$ & 1 & 3 \\
\hline \multicolumn{3}{|l|}{ Valid minimum days } \\
\hline 1 - 3 days & 7 & 3 \\
\hline 4 days & 4 & 6 \\
\hline$>4$ days & 2 & 5 \\
\hline N/I & 1 & 5 \\
\hline \multicolumn{3}{|l|}{ Epochs } \\
\hline $1-10$ seconds & 2 & 4 \\
\hline 15 seconds & 5 & 0 \\
\hline 60 seconds & 4 & 7 \\
\hline N/I & 3 & 8 \\
\hline \multicolumn{3}{|l|}{ Device model } \\
\hline ActiGraph & 11 & 14 \\
\hline $\begin{array}{l}\text { Others (GENEActiv, Actical, } \\
\text { Actiheart, SenseWear armband, } \\
\text { DynaPort and Sit-stand table) }\end{array}$ & 3 & 5 \\
\hline
\end{tabular}

Note: $\mathrm{N} / \mathrm{I}=$ not informed; $\mathrm{cpm}=$ counts per minute. period of time to which the measure referred, although 58 considered a weekday plus a weekend day, being typical or not, for evaluation of the measure. The cutoff points for SB characterization varied according to the instrument used and to the reference domains. The most commonly used being screen time, TV watching, or sitting time from $2 \mathrm{~h} /$ day $(\mathrm{n}=60)$. Another 42 studies did not specify the cut-off point.

Regarding questionnaires used in children or adolescents $(n=134)$, the first studies were from 1998 and the years with the most publications were 2015, 2016, and 2018 with 16 studies each. The sample size ranged from 12 to 109,104 individuals and the sample was composed mainly of students $(n=115)$ from the South, Southeast, and Northeast regions, with 45, 43, and 29 studies, respectively. Regarding the instruments used, 74 studies did not specify the questionnaire used, but of the most cited, 11 studies used the GSHS questionnaire. Only 49 studies presented quality indicators, specified in the study itself or in previous studies. For this age group, most of the studies considered only screen time as a measure of SB ( $n=86)$, taking as a reference a weekday plus one weekend day $(\mathrm{n}=35)$ and 2 hours as a cut-off point for SB characterization $(n=61)$.

The 64 studies that evaluated SB in adults and/ or the elderly subjectively, appeared in the year 2001, with most publications in the year $2017(n=11)$. In 24 studies, the sample was from the Southeast region, followed by 14 studies conducted in the South region. In 14 studies, it was composed only of the elderly. The most used questionnaire was the IPAQ $(n=23)$ and in 34 studies a quality indicator was presented for the instruments (either in the text itself or in previous studies). Concerning the measures, for this age group, only sitting time was most used to characterize SB (n = 29), with one weekday plus one weekend day being considered as a time reference $(n=23)$, typical or not, as a basis to record the measure. Finally, in relation to the cut-off point for SB, the majority of studies did not specify a cut-off ( $\mathrm{n}=15$ studies), with the other 11 studies considering $\geq 3 \mathrm{~h} /$ day for TV Watching.

\section{Discussion}

In this first systematic review of studies that evaluated $\mathrm{SB}$ in Brazil, we verified a predominance of subjective methods for measuring SB. Besides that, a diversity in the methods of identifying the SB manifestations also was demonstrated. As of 2013, the increase in the number of studies that evaluated SB in Brazil reveals a 
Table 2 - Main characteristics of studies that assessed sedentary behavior using subjective methods according to the sample.

\begin{tabular}{|c|c|c|}
\hline \multirow[b]{2}{*}{ Characteristics of the studies } & \multicolumn{2}{|c|}{ Number of studies } \\
\hline & $\begin{array}{c}\text { Children or } \\
\text { adolescents } \\
(\mathrm{n}=134)\end{array}$ & $\begin{array}{l}\text { Adults or elderly } \\
\qquad(\mathrm{n}=64)\end{array}$ \\
\hline \multicolumn{3}{|l|}{ Year } \\
\hline $1998-2005$ & 3 & 1 \\
\hline $2006-2010$ & 24 & 4 \\
\hline $2011-2015$ & 46 & 21 \\
\hline $2016-2020$ & 61 & 38 \\
\hline \multicolumn{3}{|l|}{ Region } \\
\hline Brazil or more than one region & 8 & 12 \\
\hline Midwest & 6 & 2 \\
\hline North & 3 & 2 \\
\hline Northeast & 29 & 10 \\
\hline South & 45 & 14 \\
\hline Southeast & 43 & 24 \\
\hline \multicolumn{3}{|l|}{ Sample size } \\
\hline$<100$ & 5 & 5 \\
\hline $100-499$ & 40 & 19 \\
\hline $500-999$ & 34 & 11 \\
\hline $1000-4999$ & 46 & 18 \\
\hline $5000-9999$ & 5 & 0 \\
\hline 10000 or more & 4 & 11 \\
\hline \multicolumn{3}{|l|}{ Questionnaire } \\
\hline GSHS & 11 & 0 \\
\hline PAQ-C & 9 & 0 \\
\hline COMPAC & 6 & 0 \\
\hline ASAQ & 5 & 0 \\
\hline Baecke Questionnaire & 5 & 0 \\
\hline IPAQ & 4 & 23 \\
\hline VIGITEL & 0 & 9 \\
\hline Others & 20 & 12 \\
\hline N/I & 74 & 20 \\
\hline \multicolumn{3}{|l|}{ Quality indicator } \\
\hline Yes & 49 & 34 \\
\hline No & 85 & 30 \\
\hline \multicolumn{3}{|l|}{$\mathrm{SB}$ indicator } \\
\hline Screen time & 86 & 10 \\
\hline Sitting time & 7 & 29 \\
\hline TV watching & 21 & 19 \\
\hline Sitting time + Screen time & 10 & 2 \\
\hline Sitting time + TV watching & 2 & 2 \\
\hline Others & 8 & 2 \\
\hline \multicolumn{3}{|l|}{ Time reference } \\
\hline Last week & 9 & 2 \\
\hline Typical day & 11 & 7 \\
\hline
\end{tabular}

\begin{tabular}{lcc}
\hline \multirow{2}{*}{ Characteristics of the studies } & \multicolumn{2}{c}{ Number of studies } \\
\cline { 2 - 3 } & $\begin{array}{c}\text { Children or } \\
\text { adolescents } \\
(\mathrm{n}=134)\end{array}$ & $\begin{array}{c}\text { Adults or elderly } \\
(\mathrm{n}=64)\end{array}$ \\
\hline Typical weekday and weekend day & 15 & 18 \\
Weekday and weekend day & 20 & 5 \\
Others & 28 & 13 \\
N/I & 51 & 19 \\
Cut-off point & & \\
Screen time $\geq 2$ h/day & 45 & 3 \\
Screen time $\geq 3$ h/day & 8 & 2 \\
Screen time $\geq 4$ h/day & 11 & 0 \\
Sitting time $2-4$ h/day & 1 & 4 \\
Sitting time $5-6$ h/day & 0 & 3 \\
Sitting time $>7$ h/day & 0 & 7 \\
TV watching $\geq 2$ h/day & 5 & 2 \\
TV watching $\geq 3$ h/day & 7 & 11 \\
TV watching $\geq 4$ h/day & 3 & 2 \\
Others & 27 & 15 \\
N/I & 27 & \\
\hline
\end{tabular}

Note: $\mathrm{N} / \mathrm{I}=$ not informed; $\mathrm{h}=$ hours; $\mathrm{TV}=$ television; GSHS = Global School-based Student Health Survey; PAQ-C = Physical Activity Questionnaire for Children; COMPAC = "Comportamentos dos adolescentes catarinenses"; ASAQ = Adolescent sedentary activity questionnaire; IPAQ = International Physical Activity Questionnaire; VIGITEL = "Vigilância de fatores de risco e proteção para doenças crônicas por inquérito telefônico".

growth in interest in this variable. In fact, in spite of the research in previous years in other countries, the first consensus on the term SB was only reached in $2012^{18}$. The South and Southeast regions presented the highest number of studies, corroborating the data of Ramires et al. ${ }^{19}$, that demonstrated that research on physical activity and SB in Brazil was concentrated in the South and Southeast regions, with growth in the Northeast region. Most of the Brazilian studies that evaluated SB used a sample composed of students. As in other countries, there is interest in researching this population because it is during the school phase that levels of physical activity begin to decrease ${ }^{20}$ and games are replaced by sedentary activities. In addition, in Brazil, the rate of attendance of children and adolescents at school, from 7 to 14 years old, is approximately $96 \%{ }^{21}$, which facilitates the access of researchers to students.

The majority of Brazilian studies (approximately $86 \%$ ) have used subjective measures to evaluate SB through self-reported questionnaires or interviews, which may compromise the quality of the measure, as these methods present less accuracy and poor correlation 
with device-derived measures ${ }^{22}$. Chastin et al. ${ }^{15}$ compared several subjective instruments of SB measurement with a device-derived measurement instrument and found that the first present great bias, low precision, and low correlation with the second, besides not presenting standardization between the domains and time reference that they aim to measure. Another point is that although accelerometers have been used in several studies to evaluate $\mathrm{SB}$, the technology of most of the models used does not allow differentiation of postural changes, considering stationary behavior as synonymous with $\mathrm{SB}$, overestimating the measurement, which should only be considered in the sitting, reclined, or lying down positions ${ }^{1}$.

On the other hand, subjective measures through questionnaires present as their main positive point the possibility of measuring different domains of sedentary behavior. In this sense, several studies conducted in different age groups have evaluated different domains of sedentary behavior and different risk indicators, such as all-cause mortality, with which only TV watching was associated, to the detriment of driving time and using the computer ${ }^{23}$. Besides that, mentally active activities do not necessarily represent a risk for some health indicators. For example, the time spent on mentally active behaviors (such as work, meetings, reading, etc.) seems not to be associated with depressive symptoms or even protection for them. In contrast, mentally passive sedentary behaviors (such as watching television, listening to music, etc.) are risk factors for depressive symptoms ${ }^{24,25}$.

Among adults, the most used questionnaire for the evaluation of SB was the IPAQ, specifically considering the final two questions about sitting time during the week and weekend. Despite the popularity and ease of access to the instrument, it was developed and is internationally recognized to assess the level of physical activity, considering SB in this context as a variable of secondary interest. Among children and adolescents, GSHS questionnaire was the most used, in agreement with other international studies, which facilitates comparisons of prevalence in this population. One question with respect to studies that use questionnaires would be the inclusion of psychometric indicators of validity and reproducibility, which are often not mentioned within the study itself, making it difficult to evaluate the quality of the instrument. In this sense, the importance of properly displaying the quality indicators and more specific SB issues is emphasized ${ }^{26}$.

Regarding the type of SB measurement, the most used was screen time, considering television, comput- ers, and video games. In other countries screen time has also been widely researched as a measure of $\mathrm{SB}^{26}$, however, it reflects only one manifestation domain of $\mathrm{SB}$, and it is necessary to specify other domains, such as hours sitting at school or work, so that the measure can be better recorded. For children and adolescents, the American Academy of Pediatrics established a cut-off point of 2 hours of screen time in $2001^{27}$. This recommendation does not include other SBs and considers other psycho-social aspects that go beyond physical sitting time. Even so, this cut-off point has been used in the majority of Brazilian studies, including those which did not only use screen time, although it is known that there is still no consensus in the literature about the amount of time spent on SB that is harmful to health ${ }^{28}$.

Also, regarding subjective measures, there is great variability in relation to the reference time of the question involving the $\mathrm{SB}$ measurement, which makes it difficult to compare the studies for epidemiological surveys that could determine the prevalence of $\mathrm{SB}$ in the Brazilian population. However, it is still unclear in the literature how best to measure subjective SB. Depending on the purpose of the research, whether for surveillance or epidemiological surveys, in all domains or specific domains, the manner used to ask the question or the reference time can vary greatly ${ }^{15}$.

The diversity of instruments for SB evaluation found in Brazilian studies can also be observed in populations from other countries. A systematic review, conducted by Dall et al. ${ }^{29}$ with studies worldwide, found 141 different questions to assess SB, totaling 32 instruments, and this only for the adult and elderly population. After the review, the authors proposed a taxonomy to help in choosing the most appropriate tool, according to the domain and period of time that the measure intended to evaluate.

To the best of our knowledge, the present review is the first to survey Brazilian studies that evaluated $\mathrm{SB}$ and demonstrate the main characteristics of these studies, having as a strong point many articles included, which provided a lot of data on the form of evaluation of SB in Brazil. For example, although inclinometers are considered the best instruments for SB evaluation, because of their precision regarding postural transitions ${ }^{30}$, no Brazilian study has used this tool. The novelty and high cost of these devices are still a barrier to the use in research from middle-income countries such as Brazil.

On the other hand, as the main limitation, we considered many studies where the focus was not the eval- 
uation of SB, but rather the level of physical activity, which made it difficult to analyze the quality criteria of the instruments in relation to SB alone. Therefore, the majority of Brazilian studies that evaluated SB used subjective measurement instruments, such as questionnaires, without presenting explicit quality indicators such as reproducibility and validity. In addition, different types of questionnaires, different cut-off points, and different time references were found for SB estimation. In this way, public policies that aim to reduce SB should consider the available studies with caution, considering possible biases due to the instruments used.

\section{Conflict of interest}

The authors declare no conflict of interest.

\section{Author's contributions}

Barboza LLS participated in the conception, design, analysis, data interpretation, and writing of the article; Silva ECM, Thuany M, Araujo RHO, and Werneck $\mathrm{AO}$ participated in the analysis, data interpretation, article writing, and relevant critical review of the intellectual content; Oliveira DN and Gandarela L participated in the analysis, data interpretation and relevant critical review of the intellectual content; Silva DRP participated in the conception, design, analysis, data interpretation, and relevant critical review of intellectual content. All authors approved the final version to be published.

\section{Acknowledgements}

Silva ECM and Araujo RHO are supported by the Coordination for the Improvement of Higher Education Personnel (CAPES) with a PhD scholarship (CAPES Process: 88887.605029 / 202100 and 88887.605034 / 2021-00). Werneck AO is supported by the São Paulo Research Foundation (FAPESP) with a $\mathrm{PhD}$ scholarship (FAPESP process: 2019 / 24124-7). The views expressed in this publication are those of the authors and not necessarily those of the acknowledged institutions.

\section{References}

1. Tremblay MS, Aubert S, Barnes JD, Saunders TJ, Carson $\mathrm{V}$, Latimer-Cheung AE et al. Sedentary Behavior Research Network (SBRN) - Terminology Consensus Project process and outcome. Int J Behav Nutr Phys Act. 2017;14(1):1-17.

2. Loyen A, Chau JY, Jelsma JGM, van Nassau F, van der Ploeg HP. Prevalence and correlates of domain-specific sedentary time of adults in the Netherlands: findings from the 2006 Dutch time use survey. BMC Public Health. 2019;19(2):1-12.

3. Stamatakis E, Ekelund U, Ding D, Hamer M, Bauman AE, Lee I-M. Is the time right for quantitative public health guidelines on sitting? A narrative review of sedentary behaviour research paradigms and findings. $\mathrm{Br} \mathrm{J}$ Sports Med. 2019;53(6):377-82.
4. Pandey A, Salahuddin U, Garg S, Ayers C, Kulinski J, Anand $\mathrm{V}$ et al. Continuous Dose-Response Association Between Sedentary Time and Risk for Cardiovascular Disease: A Meta-analysis. JAMA Cardiol. 2016;1(5):575-83.

5. Tremblay MS, Colley RC, Saunders TJ, Healy GN, Owen N. Physiological and health implications of a sedentary lifestyle. Appl Physiol Nutr Metab. 2010;35(6):725-40.

6. Rezende LFM, Rodrigues Lopes M, Rey-López JP, Matsudo VKR, Luiz O do C. Sedentary behavior and health outcomes: an overview of systematic reviews. PloS One. 2014;9(8):e105620.

7. Bull FC, Al-Ansari SS, Biddle S, Borodulin K, Buman MP, Cardon $\mathrm{G}$ et al. World Health Organization 2020 guidelines on physical activity and sedentary behaviour. Br J Sports Med. 2020;54(24):1451-62.

8. Ross R, Chaput J-P, Giangregorio LM, Janssen I, Saunders TJ, Kho ME et al. Canadian 24-Hour Movement Guidelines for Adults aged 18-64 years and Adults aged 65 years or older: an integration of physical activity, sedentary behaviour, and sleep. Appl Physiol Nutr Metab. 2020;45(10):S57-102.

9. Tremblay MS, Carson V, Chaput J-P, Connor Gorber S, Dinh T, Duggan $M$ et al. Canadian 24-Hour Movement Guidelines for Children and Youth: An Integration of Physical Activity, Sedentary Behaviour, and Sleep. Appl Physiol Nutr Metab. 2016;41(6):S311-27.

10. Virgara R, Phillips A, Lewis L, Richardson M, Maher C. Development of Australian physical activity and screen time guidelines for outside school hours care: an international Delphi study. Int J Behav Nutr Phys Act. 2021;18(1):1-14.

11. Okely AD, Ghersi D, Hesketh KD, Santos R, Loughran SP, Cliff DP et al. A collaborative approach to adopting/adapting guidelines - The Australian 24-Hour Movement Guidelines for the early years (Birth to 5 years): an integration of physical activity, sedentary behavior, and sleep. BMC Public Health. 2017;17(5):167-90.

12. Atkin AJ, Gorely T, Clemes SA, Yates T, Edwardson C, Brage $\mathrm{S}$ et al. Methods of Measurement in epidemiology: sedentary Behaviour. Int J Epidemiol. 2012;41(5):1460-71.

13. Owen N, Healy GN, Dempsey PC, Salmon J, Timperio A, Clark BK et al. Sedentary Behavior and Public Health: Integrating the Evidence and Identifying Potential Solutions. Annu Rev Public Health. 2020;41:265-87.

14. Bakker EA, Hartman YAW, Hopman MTE, Hopkins ND, Graves LEF, Dunstan DW et al. Validity and reliability of subjective methods to assess sedentary behaviour in adults: a systematic review and meta-analysis. Int J Behav Nutr Phys Act. 2020;17(1):1-31.

15. Chastin SFM, Dontje ML, Skelton DA, Čukić I, Shaw RJ, Gill JMR et al. Systematic comparative validation of self-report measures of sedentary time against an objective measure of postural sitting (activPAL). Int J Behav Nutr Phys Act. 2018; 15(1):1-12.

16. Prince SA, Cardilli L, Reed JL, Saunders TJ, Kite C, Douillette $\mathrm{K}$ et al. A comparison of self-reported and device measured sedentary behaviour in adults: a systematic review and metaanalysis. Int J Behav Nutr Phys Act. 2020;17(1):1-17.

17. Moher D, Liberati A, Tetzlaff J, Altman DG, PRISMA Group. Preferred reporting items for systematic reviews and meta-analyses: the PRISMA statement. PLoS Med. 2009;6(7):e1000097.

18. Bames J, Behrens TK, Benden ME, Biddle S, Bond D, Brassard $\mathrm{P}$ et al. Letter to the editor: standardized use of the terms 'sedentary' and 'sedentary behaviours'. Appl Physiol Nutr Metab. 2012;37(3):540-2. 
19. Ramires V, Becker L, Sadovsky A, Zago A, Bielemann R, Guerra P. Evolução da pesquisa epidemiológica em atividade física e comportamento sedentário no Brasil: atualização de uma revisão sistemática. Rev Bras Ativ Fis Saude. 2014;19(5):529-47.

20. Reilly JJ. When does it all go wrong? Longitudinal studies of changes in moderate-to-vigorous-intensity physical activity across childhood and adolescence. J Exerc Sci Fit. 2016;14(1):1-6.

21. INEP. Estatísticas da educação básica no Brasil: 2019. Brasília: INEP; 2019. Available in <http://inep.gov.br/ sinopses-estatisticas-da-educacao-basica > [2021 Julho].

22. Matthews CE, Kozey Keadle S, Moore SC, Schoeller DS, Carroll RJ, Troiano RP et al. Measurement of Active and Sedentary Behavior in Context of Large Epidemiologic Studies. Med Sci Sports Exerc. 2018;50(2):266-76.

23. Basterra-Gortari FJ, Bes-Rastrollo M, Gea A, NúñezCórdoba JM, Toledo E, Martínez-González MÁ. Television viewing, computer use, time driving and all-cause mortality: the SUN cohort. J Am Heart Assoc. 2014;3(3):e000864.

24. Kikuchi H, Inoue S, Sugiyama T, Owen N, Oka K, Nakaya $\mathrm{T}$ et al. Distinct associations of different sedentary behaviors with health-related attributes among older adults. Prev Med. 2014;67:335-9.

25. Hallgren M, Owen N, Stubbs B, Zeebari Z, Vancampfort $\mathrm{D}$, Schuch $\mathrm{F}$ et al. Passive and mentally-active sedentary behaviors and incident major depressive disorder: A 13-year cohort study. J Affect Disord. 2018;241:579-85.
26. Prince SA, LeBlanc AG, Colley RC, Saunders TJ. Measurement of sedentary behaviour in population health surveys: a review and recommendations. PeerJ. 2017;5:e4130.

27. American Academy of Pediatrics. Committee on Public Education. American Academy of Pediatrics: Children, adolescents, and television. Pediatrics. 2001;107(2):423-6.

28. Magnon V, Dutheil F, Auxiette C. Sedentariness: A Need for a Definition. Front Public Health. 2018;6:372.

29. Dall PM, Coulter EH, Fitzsimons CF, Skelton DA, Chastin S, Seniors USP Team. TAxonomy of Self-reported Sedentary behaviour Tools (TASST) framework for development, comparison and evaluation of self-report tools: content analysis and systematic review. BMJ Open. 2017;7(4):e013844.

30. Grant PM, Ryan CG, Tigbe WW, Granat MH. The validation of a novel activity monitor in the measurement of posture and motion during everyday activities. Br J Sports Med. 2006;40(12):992-7.

Received: 24/08//2021

Approved: 12/12/2021

\section{Quote this article as:}

Barboza LLS, Silva ECM, Thuany M, Araujo RHO, Werneck AO, Oliveira DN, Gandarela L, Silva DRP. Approaches and methods to assess sedentary behavior in Brazil: a systematic review. Rev Bras Ativ Fis Saúde. 2021;26:e0236. DOI: 10.12820/rbafs.26e0236 
Supplementary Table 1 - Main characteristics of studies that assessed sedentary behavior using device-derived methods.

\begin{tabular}{|c|c|c|c|c|c|c|c|c|c|c|}
\hline Author (year) & Region & City-State or State & $\mathrm{n}$ & Sample & $\begin{array}{l}\text { Days } \\
\text { of } \\
\text { use }\end{array}$ & $\begin{array}{l}\text { Minimum } \\
\text { hours of } \\
\text { use }\end{array}$ & Cut-off points & $\begin{array}{l}\text { Valid } \\
\text { minimum } \\
\text { days }\end{array}$ & Epochs & Device model \\
\hline $\begin{array}{l}\text { Ferrari et al. } \\
(2020)^{1}\end{array}$ & Brazil & Brazil & 524 & Adults $18-65$ y & 7 & 10 & $<100 \mathrm{cpm}$ & $\begin{array}{l}5 \text { week } \\
\text { days and } 1 \\
\text { weekend } \\
\text { day }\end{array}$ & $60 \mathrm{~s}$ & ActiGraph GT3X+ \\
\hline $\begin{array}{l}\text { Barbieri et al. } \\
(2017)^{2}\end{array}$ & Brazil & Brazil & 24 & $\begin{array}{l}\text { Office workers } \\
\pm 41.3 \text { y }\end{array}$ & 60 & $\mathrm{x}$ & $\mathrm{x}$ & $\mathrm{x}$ & $\mathrm{x}$ & Sit-stand table \\
\hline $\begin{array}{l}\text { Sasaki et al. } \\
(2018)^{3}\end{array}$ & Brazil & Brazil & 42 & $\begin{array}{l}\text { Elderly } 65- \\
75 \text { ys }\end{array}$ & 7 & 10 & $\begin{array}{l}<100 \mathrm{cpm} \text { and } \\
<200 \mathrm{cpm}\end{array}$ & 7 & $60 \mathrm{~s}$ & $\begin{array}{l}\text { ActiGraph } \\
\text { wGT3X-BT }\end{array}$ \\
\hline $\begin{array}{l}\text { Mendonça et } \\
\text { al. }(2018)^{4}\end{array}$ & Northeast & $\begin{array}{l}\text { João Pessoa, } \\
\text { Paraíba }\end{array}$ & 656 & $\begin{array}{l}\text { Students } 10- \\
14 \mathrm{y}\end{array}$ & 7 & 8 & $<100 \mathrm{cpm}$ & 3 & $60 \mathrm{~s}$ & Actigraph GT3X+ \\
\hline $\begin{array}{l}\text { Santos et al. } \\
(2019)^{5}\end{array}$ & Northeast & $\begin{array}{l}\text { Natal, Rio Grande } \\
\text { do Norte }\end{array}$ & 17 & $\begin{array}{l}\text { Obese Adults } \pm \\
30.2 \mathrm{y}\end{array}$ & 7 & 10 & $<100 \mathrm{cpm}$ & $\begin{array}{l}4 \text { week } \\
\text { days and } 1 \\
\text { weekend } \\
\text { day }\end{array}$ & $60 \mathrm{~s}$ & ActiGraph GT9X \\
\hline $\begin{array}{l}\text { Gerage et al. } \\
(2015)^{6}\end{array}$ & Northeast & $\begin{array}{l}\text { Recife, } \\
\text { Pernambuco }\end{array}$ & 87 & $\begin{array}{l}\text { Hypertensive } \\
\text { patients }>40 \mathrm{y}\end{array}$ & 7 & 10 & $<100 \mathrm{cpm}$ & 4 & $60 \mathrm{~s}$ & $\begin{array}{l}\text { ActiGraph GT3X } \\
\text { and GT3X+ }\end{array}$ \\
\hline $\begin{array}{l}\text { Melo et al. } \\
(2018)^{7}\end{array}$ & Northeast & Sergipe State & 100 & $\begin{array}{l}\text { Children and } \\
\text { adolescents } \\
\text { patients with } \\
\text { SCA }\end{array}$ & 7 & 10 & $<100 \mathrm{cpm}$ & 7 & $60 \mathrm{~s}$ & $\begin{array}{l}\text { ActiGraph } \\
\text { wGT3X-BT }\end{array}$ \\
\hline $\begin{array}{l}\text { Gonçalves et } \\
\text { al. }(2017)^{8}\end{array}$ & South & Curitiba, Paraná & 305 & Adults $20-65$ y & 7 & 10 & $<100 \mathrm{cpm}$ & 5 & $60 \mathrm{~s}$ & $\begin{array}{l}\text { ActiGraph } 7164 \\
\text { and GT1M }\end{array}$ \\
\hline $\begin{array}{l}\text { Bacil et al. } \\
(2018)^{9}\end{array}$ & South & Curitiba, Paraná & 117 & $\begin{array}{l}\text { Students } 9 \text { - } \\
15 \mathrm{y}\end{array}$ & 7 & 8 & $<100 \mathrm{cpm}$ & 4 & $10 \mathrm{~s}$ & ActiGraph GT3X \\
\hline $\begin{array}{l}\text { Santos et al. } \\
(2018)^{10}\end{array}$ & South & $\begin{array}{l}\text { Florianópolis, } \\
\text { Santa Catarina }\end{array}$ & 425 & Elderly $\geq 63 \mathrm{y}$ & 7 & $\begin{array}{l}10 \text { week } \\
\text { days and } 8 \\
\text { weekend } \\
\text { days }\end{array}$ & $<100 \mathrm{cpm}$ & 4 & $6 \mathrm{~s}$ & $\begin{array}{l}\text { ActiGraph GT3X } \\
\text { and GT3X+ }\end{array}$ \\
\hline $\begin{array}{l}\text { Costa et al. } \\
(2017)^{11}\end{array}$ & South & $\begin{array}{l}\text { Florianópolis, } \\
\text { Santa Catarina }\end{array}$ & 571 & $\begin{array}{l}\text { Students } 7 \text { - } \\
12 \mathrm{y}\end{array}$ & 2 & $\mathrm{x}$ & $<100 \mathrm{cpm}$ & 1 & $15 \mathrm{~s}$ & Actigraph GT3X+ \\
\hline $\begin{array}{l}\text { Martins et al. } \\
(2019)^{12}\end{array}$ & South & $\begin{array}{l}\text { Florianópolis, } \\
\text { Santa Catarina }\end{array}$ & 64 & $\begin{array}{l}\text { Children and } \\
\text { adolescents } \\
\text { infected with } \\
\text { HIV } 8 \text { - } 15 \text { y }\end{array}$ & 7 & 10 & $<100 \mathrm{cpm}$ & 4 & $\mathrm{x}$ & $\begin{array}{l}\text { ActiGraph GT3X- } \\
\text { Plus }\end{array}$ \\
\hline $\begin{array}{l}\text { Furlanetto et } \\
\text { al. }(2017)^{13}\end{array}$ & South & Londrina, Paraná & 101 & $\begin{array}{l}\text { Patients with } \\
\text { COPD } 59 \text { - } \\
74 \text { y }\end{array}$ & 2 & 12 & $\begin{array}{l}<1.5 \mathrm{MET} \\
\text { and }<2 \mathrm{MET}\end{array}$ & 2 & $\mathrm{x}$ & $\begin{array}{l}\text { SenseWear } \\
\text { armband and } \\
\text { Triaxial DynaPort } \\
\text { activity monitor }\end{array}$ \\
\hline $\begin{array}{l}\text { Barbosa et al. } \\
(2016)^{14}\end{array}$ & South & Londrina, Paraná & 370 & $\begin{array}{l}\text { Preschoolers } \\
4-6 y\end{array}$ & 5 & 2 & $\begin{array}{l}\text { Sirard et al. } \\
\text { for children } 4 \\
-5 \text { y and Van } \\
\text { Cauwenbrghe } \\
\text { et al. for } \\
\text { children } 6 \text { y }\end{array}$ & 3 & 1s & ActiGraph GT3X \\
\hline $\begin{array}{l}\text { Ramos et al. } \\
(2018)^{15}\end{array}$ & South & Londrina, Paraná & 394 & Students & 7 & 8 & $\begin{array}{l}180 \\
\text { counts/15s }\end{array}$ & 4 & $15 \mathrm{~s}$ & $\begin{array}{l}\text { ActiGraph GT3X } \\
\text { and GT3X-Plus }\end{array}$ \\
\hline $\begin{array}{l}\text { Silva et al. } \\
(2014)^{16}\end{array}$ & South & Londrina, Paraná & 79 & $\begin{array}{l}\text { Students } 10- \\
15 \mathrm{y}\end{array}$ & 1 & $\mathrm{x}$ & $\begin{array}{l}<100 \mathrm{cpm} \text { and } \\
<1.5 \mathrm{MET}\end{array}$ & 1 & $60 \mathrm{~s}$ & $\begin{array}{l}\text { Acelerometry by } \\
\text { Actical and indirect } \\
\text { calorimetry by } \\
\text { Cosmed Model } \\
\text { K4b2 }\end{array}$ \\
\hline $\begin{array}{l}\text { Mielke et al. } \\
(2018)^{17}\end{array}$ & South & $\begin{array}{l}\text { Pelotas, Rio } \\
\text { Grande do Sul }\end{array}$ & 4106 & Adults $18 \mathrm{y}$ & 7 & 24 & $\begin{array}{l}<12.7 \text { hours/ } \\
\text { day }\end{array}$ & 2 & $5 \mathrm{~s}$ & GENEActiv \\
\hline $\begin{array}{l}\text { Barbosa } \\
\text { Porcellis da } \\
\text { Silva, Marques } \\
\text { and Reichert } \\
(2018)^{18}\end{array}$ & South & $\begin{array}{l}\text { Pelotas, Rio } \\
\text { Grande do Sul }\end{array}$ & 90 & $\begin{array}{l}\text { Adults } \\
\text { with visual } \\
\text { impairment } 18 \\
\text { - } 95 \mathrm{y}\end{array}$ & 7 & 8 & $<100 \mathrm{cpm}$ & 3 & $5 \mathrm{~s}$ & $\begin{array}{l}\text { ActiGraph } \\
\text { wGT3xp }\end{array}$ \\
\hline
\end{tabular}

Continue... 
Continuation of Supplementary Table 1 - Main characteristics of studies that assessed sedentary behavior using device-derived methods.

\begin{tabular}{|c|c|c|c|c|c|c|c|c|c|c|}
\hline Author (year) & Region & City-State or State & $\mathrm{n}$ & Sample & $\begin{array}{l}\text { Days } \\
\text { of } \\
\text { use }\end{array}$ & $\begin{array}{l}\text { Minimum } \\
\text { hours of } \\
\text { use }\end{array}$ & Cut-off points & $\begin{array}{l}\text { Valid } \\
\text { minimum } \\
\text { days }\end{array}$ & Epochs & Device model \\
\hline $\begin{array}{l}\text { Horta et al. } \\
(2015)^{19}\end{array}$ & South & $\begin{array}{l}\text { Pelotas, Rio } \\
\text { Grande do Sul }\end{array}$ & 1241 & Adults & 7 & 10 & $\begin{array}{l}<50 \\
\text { milligrams }\end{array}$ & 4 & $5 \mathrm{~s}$ & GENEActiv \\
\hline $\begin{array}{l}\text { Faria et al. } \\
(2020)^{20}\end{array}$ & Southeast & $\begin{array}{l}\text { Ituiutaba, Minas } \\
\text { Gerais }\end{array}$ & 217 & $\begin{array}{l}\text { Students } 15- \\
18 \mathrm{y}\end{array}$ & 8 & 10 & $\begin{array}{l}75 \text { th } \\
\text { percentile }\end{array}$ & $\begin{array}{l}6 \text { week } \\
\text { days and } 1 \\
\text { weekend } \\
\text { day }\end{array}$ & $15 \mathrm{~s}$ & ActiGraph GT3X \\
\hline $\begin{array}{l}\text { Gomes et al. } \\
(2015)^{21}\end{array}$ & Southeast & $\begin{array}{l}\text { Juiz de Fora, } \\
\text { Minas Gerais }\end{array}$ & 19 & $\begin{array}{l}\text { Patients on } \\
\text { hemodialysis } \\
18-65 y\end{array}$ & 4 & 12 & $\begin{array}{l}<5.000 \text { steps/ } \\
\text { day }\end{array}$ & $\mathrm{x}$ & $\mathrm{x}$ & DynaPortMiniMod \\
\hline $\begin{array}{l}\text { Lima-Junior } \\
\text { et al. }(2019)^{22}\end{array}$ & Southeast & $\begin{array}{l}\text { Minas Gerais } \\
\text { State }\end{array}$ & 153 & $\begin{array}{l}\text { Students } 10- \\
12 \mathrm{y}\end{array}$ & 3 & $\mathrm{x}$ & $<1.6 \mathrm{MET}$ & 3 & $\mathrm{x}$ & $\begin{array}{l}\text { Actiheart developed } \\
\text { by MiniMitter }\end{array}$ \\
\hline $\begin{array}{l}\text { Paravidino et } \\
\text { al. }(2017)^{23}\end{array}$ & Southeast & $\begin{array}{l}\text { Niterói, Rio de } \\
\text { Janeiro }\end{array}$ & 24 & $\begin{array}{l}\text { Overweight } \\
\text { boys } 11-13 \text { y }\end{array}$ & 7 & 10 & $\mathrm{x}$ & $\mathrm{x}$ & $\mathrm{x}$ & Actical \\
\hline $\begin{array}{l}\text { Crisp et al. } \\
(2018)^{24}\end{array}$ & Southeast & $\begin{array}{l}\text { Piracicaba, São } \\
\text { Paulo }\end{array}$ & 42 & $\begin{array}{l}\text { Female } \\
\text { candidates for } \\
\text { bariatric surgery } \\
20-40 \text { y }\end{array}$ & 7 & 10 & $<100 \mathrm{cpm}$ & $\mathrm{x}$ & $\mathrm{x}$ & ActiGraph GT3X+ \\
\hline $\begin{array}{l}\text { Diniz et al. } \\
(2017)^{25}\end{array}$ & Southeast & $\begin{array}{l}\text { Presidente } \\
\text { Prudente, São } \\
\text { Paulo }\end{array}$ & 49 & $\begin{array}{l}\text { Postmenopausal } \\
\text { women > 50y }\end{array}$ & 7 & 10 & $<100 \mathrm{cpm}$ & 5 & $60 s$ & ActiGraph GT3X \\
\hline $\begin{array}{l}\text { Moura et al. } \\
(2019)^{26}\end{array}$ & Southeast & $\begin{array}{l}\text { Rio Pomba, Minas } \\
\text { Gerais }\end{array}$ & 84 & $\begin{array}{l}\text { Students } 14- \\
18 \mathrm{y}\end{array}$ & 7 & 8 & $\begin{array}{l}391.8 \\
\min \cdot d a y-1\end{array}$ & 3 & $15 \mathrm{~s}$ & Actigraph GT3X+ \\
\hline $\begin{array}{l}\text { Sperandio et } \\
\text { al. }(2017)^{27}\end{array}$ & Southeast & Santos, São Paulo & 553 & Adults $\geq 20 \mathrm{y}$ & 7 & 10 & $<100 \mathrm{cpm}$ & 4 & $\mathrm{x}$ & Actigraph GT3X+ \\
\hline $\begin{array}{l}\text { Ferrari et al. } \\
(2015)^{28}\end{array}$ & Southeast & $\begin{array}{l}\text { São Caetano do } \\
\text { Sul, São Paulo }\end{array}$ & 485 & $\begin{array}{l}\text { Students } 9- \\
11 \mathrm{y}\end{array}$ & 7 & 10 & $\begin{array}{l}\leq 25 \\
\text { counts } / 15 \mathrm{~s}\end{array}$ & 4 & $15 \mathrm{~s}$ & Actigraph GT3X+ \\
\hline $\begin{array}{l}\text { Lauria et al. } \\
(2017)^{29}\end{array}$ & Southeast & São Paulo State & 66 & $\begin{array}{l}\text { Smoking and } \\
\text { non-smoking } \\
\text { adults }\end{array}$ & 7 & $\mathrm{x}$ & $\mathrm{x}$ & $\mathrm{x}$ & $\mathrm{x}$ & ActiGraph GT3X \\
\hline $\begin{array}{l}\text { Gerage et al. } \\
(2019)^{30}\end{array}$ & Southeast & $\begin{array}{l}\text { São Paulo, São } \\
\text { Paulo }\end{array}$ & 174 & $\begin{array}{l}\text { Peripheral } \\
\text { Artery Disease } \\
\text { Patients } \pm \\
66,7 \mathrm{y}\end{array}$ & 7 & 10 & $<100 \mathrm{cpm}$ & 4 & $60 s$ & ActiGraph GT3X+ \\
\hline $\begin{array}{l}\text { Xavier et al. } \\
(2019)^{31}\end{array}$ & Southeast & $\begin{array}{l}\text { São Paulo, São } \\
\text { Paulo }\end{array}$ & 152 & $\begin{array}{l}\text { Pacients adults } \\
\text { and elderly with } \\
\text { COPD }\end{array}$ & 6 & $\mathrm{x}$ & $\mathrm{x}$ & 6 & $\mathrm{x}$ & ActiGraph GT3X+ \\
\hline $\begin{array}{l}\text { Moreno et al. } \\
(2019)^{32}\end{array}$ & Southeast & $\begin{array}{l}\text { São Paulo, São } \\
\text { Paulo }\end{array}$ & 68 & $\begin{array}{l}\text { Hospitalised } \\
\text { patients } 60 \mathrm{y}\end{array}$ & $\mathrm{x}$ & $\mathrm{x}$ & $<100 \mathrm{cpm}$ & $\mathrm{x}$ & $\mathrm{x}$ & ActiGraph GT3X \\
\hline $\begin{array}{l}\text { Caetano et al. } \\
(2016)^{33}\end{array}$ & Southeast & $\begin{array}{l}\text { Viçosa, Minas } \\
\text { Gerais }\end{array}$ & 101 & Students $10 \mathrm{y}$ & 3 & $\mathrm{x}$ & $<150 \mathrm{cpm}$ & 3 & $60 s$ & ActiGraph GT3X \\
\hline
\end{tabular}

Note: $\mathrm{y}=$ years; $\mathrm{SCA}=$ sickle cell disease HIV = human immunodeficiency virus; $\mathrm{COPD}=$ chronic obstructive pulmonary disease; $\mathrm{cpm}=$ counts per minute; $\mathrm{MET}=$ metabolic equivalente task. 
Supplementary Table 2 - Main characteristics of studies that assessed sedentary behavior using subjective methods in adults or elderly.

\begin{tabular}{|c|c|c|c|c|c|c|c|c|c|}
\hline Author (year) & Region & City-State or State & $\mathrm{n}$ & Sample & $\begin{array}{l}\text { Questionnaire } \\
\text { used }\end{array}$ & $\begin{array}{l}\text { Quality } \\
\text { Indicator? }\end{array}$ & SB indicator & $\begin{array}{l}\text { Time } \\
\text { reference }\end{array}$ & $\begin{array}{l}\text { Cut-off } \\
\text { point }\end{array}$ \\
\hline $\begin{array}{l}\text { Fares et al. } \\
(2012)^{34}\end{array}$ & Brazil & $\begin{array}{l}\text { Antônio Carlos, } \\
\text { Santa Catarina } \\
\text { and Lafaiete } \\
\text { Coutinho, Bahia }\end{array}$ & 659 & Elderly $\geq 60 y$ & IPAQ & Yes & Sitting time & $\begin{array}{l}\text { Typical } \\
\text { weekday and } \\
\text { weekend day }\end{array}$ & $\geq 6 \mathrm{~h} / \mathrm{day}$ \\
\hline $\begin{array}{l}\text { Schuch et al. } \\
(2020)^{35}\end{array}$ & Brazil & Brazil & 937 & Adults $\geq 18 y$ & $\mathrm{x}$ & No & Sitting time & $\begin{array}{l}\text { Since self- } \\
\text { isolating }\end{array}$ & $\geq 10 \mathrm{~h} /$ day \\
\hline $\begin{array}{l}\text { Alves et al. } \\
(2017)^{36}\end{array}$ & Brazil & Brazil & 423 & $\begin{array}{l}\text { Shift workers } \\
18-60 y\end{array}$ & IPAQ & Yes & Sitting time & $\begin{array}{l}\text { Typical } \\
\text { weekday and } \\
\text { weekend day }\end{array}$ & $\mathrm{x}$ \\
\hline $\begin{array}{l}\text { Azevedo Barros } \\
\text { et al. }(2016)^{37}\end{array}$ & Brazil & Brazil & 49025 & Adults $20-59 y$ & PNS & No & TV watching & $\mathrm{x}$ & $\geq 5 \mathrm{~h} /$ day \\
\hline $\begin{array}{l}\text { Malta et al. } \\
(2020)^{38}\end{array}$ & Brazil & Brazil & 45161 & Adults $\geq 18 \mathrm{y}$ & $\begin{array}{l}\text { ConVid } \\
\text { Behavior } \\
\text { Survey }\end{array}$ & No & Screen time & $\begin{array}{l}\text { Before the } \\
\text { pandemic } \\
\text { and during } \\
\text { the pandemic }\end{array}$ & $\begin{array}{l}\text { Average } \\
\text { point of TV } \\
\text { time }\end{array}$ \\
\hline $\begin{array}{l}\text { Vega, Poblacion } \\
\text { and Taddei } \\
(2015)^{39}\end{array}$ & Brazil & Brazil & 2881 & $\begin{array}{l}\text { Women } 15 \text { - } \\
49 \mathrm{y}\end{array}$ & $\begin{array}{l}\text { "Pesquisa } \\
\text { Nacional de } \\
\text { Demografia } \\
\text { e Saúde da } \\
\text { Criança e da } \\
\text { Mulher" }\end{array}$ & No & TV watching & $\mathrm{x}$ & $\begin{array}{l}\text { Every day } \\
\text { and almost } \\
\text { every day }\end{array}$ \\
\hline $\begin{array}{l}\text { Mielke et al. } \\
(2014)^{40}\end{array}$ & Brazil & Brazil & 371271 & Adults $\geq 18 \mathrm{y}$ & VIGITEL & No & TV watching & Typical day & $>3 \mathrm{~h} /$ day \\
\hline $\begin{array}{l}\text { Garcia et al. } \\
(2014)^{41}\end{array}$ & Brazil & Brazil & 47477 & Workers & $\mathrm{x}$ & Yes & $\begin{array}{l}\text { TV watching } \\
\text { and sedentary } \\
\text { activities in } \\
\text { work and } \\
\text { transport }\end{array}$ & $\begin{array}{l}\text { Typical } \\
\text { weekday }\end{array}$ & $\geq 4 \mathrm{~h} /$ day \\
\hline $\begin{array}{l}\text { Cortes et al. } \\
(2013)^{42}\end{array}$ & Brazil & Brazil & 13262 & $\begin{array}{l}\text { Adult women } \\
18-49 y\end{array}$ & $\begin{array}{l}\text { Demographic } \\
\text { Health Survey }\end{array}$ & No & TV watching & Typical week & $\begin{array}{l}\geq 5 \text { times/ } \\
\text { week }\end{array}$ \\
\hline $\begin{array}{l}\text { Knuth et al. } \\
(2011)^{43}\end{array}$ & Brazil & Brazil & 292553 & $\begin{array}{l}\text { Adolescents and } \\
\text { adults } \geq 14 \mathrm{y}\end{array}$ & PNAD & No & TV watching & $\mathrm{x}$ & $\geq 3 \mathrm{~h} /$ day \\
\hline $\begin{array}{l}\text { Epifânio et al. } \\
(2020)^{44}\end{array}$ & Brazil & Brazil & 410684 & Adults > $18 \mathrm{y}$ & VIGITEL & No & TV watching & $\mathrm{x}$ & $>3 \mathrm{~h} /$ day \\
\hline $\begin{array}{l}\text { Pitanga et al. } \\
(2018)^{45}\end{array}$ & Brazil & $\begin{array}{l}\text { Salvador, Vitória, } \\
\text { Belo Horizonte, } \\
\text { Rio de Janeiro, São } \\
\text { Paulo and Porto } \\
\text { Alegre }\end{array}$ & 13765 & $\begin{array}{l}\text { Active or retired } \\
\text { civil servants } 35 \\
-74 \text { y }\end{array}$ & $\mathrm{x}$ & No & Sitting time & $\mathrm{x}$ & $\geq 8 \mathrm{~h} /$ day \\
\hline $\begin{array}{l}\text { Cassia Spanhol } \\
\text { and Bucalen- } \\
\text { Ferrari }(2016)^{46}\end{array}$ & Midwest & $\begin{array}{l}\text { Barra das Garças, } \\
\text { Mato Grosso }\end{array}$ & 305 & Adults & $\mathrm{x}$ & No & Screen time & $\mathrm{x}$ & $\mathrm{x}$ \\
\hline $\begin{array}{l}\text { Santos et al. } \\
(2019)^{47}\end{array}$ & Midwest & $\begin{array}{l}\text { Brasília, Distrito } \\
\text { Federal }\end{array}$ & 35 & Male 18 - 40 y & IPAQ & Yes & Sitting time & typical week & $\mathrm{x}$ \\
\hline $\begin{array}{l}\text { Mielke et al. } \\
(2015)^{48}\end{array}$ & North & North region & 104168 & Adults $\geq 18 \mathrm{y}$ & VIGITEL & No & TV watching & Typical day & $\geq 3 \mathrm{~h} /$ day \\
\hline $\begin{array}{l}\text { Moretti et al. } \\
(2014)^{49}\end{array}$ & North & Rio Branco, Acre & 1104 & College students & $\mathrm{x}$ & No & Screen time & $\begin{array}{l}\text { Typical } \\
\text { weekday }\end{array}$ & $\begin{array}{l}\text { Watch TV } \\
\text { every day }\end{array}$ \\
\hline $\begin{array}{l}\text { Silva et al. } \\
(2018)^{50}\end{array}$ & Northeast & Alcobaça, Bahia & 457 & Elderly $\geq 60 y$ & IPAQ & Yes & Sitting time & $\begin{array}{l}\text { Typical } \\
\text { weekday and } \\
\text { weekend day }\end{array}$ & $\begin{array}{l}\text { males: > } \\
495 \mathrm{~min} / \\
\text { day; female: } \\
\text { > } 536 \mathrm{~min} / \\
\text { day }\end{array}$ \\
\hline $\begin{array}{l}\text { Silva et al. } \\
(2012)^{51}\end{array}$ & Northeast & Aracaju, Sergipe & 298 & Women $\geq 50 \mathrm{y}$ & IPAQ & Yes & Sitting time & $\begin{array}{l}\text { Typical } \\
\text { weekday and } \\
\text { weekend day }\end{array}$ & $\mathrm{x}$ \\
\hline $\begin{array}{l}\text { Smith-Menezes } \\
\text { et al. }(2012)^{52}\end{array}$ & Northeast & Aracaju, Sergipe & 758 & Military 18 y & $\mathrm{x}$ & No & Screen time & $\mathrm{x}$ & $\geq 2 \mathrm{~h} /$ day \\
\hline
\end{tabular}

Continue... 
Continuation of Supplementary Table 2 - Main characteristics of studies that assessed sedentary behavior using subjective methods in adults or elderly.

\begin{tabular}{|c|c|c|c|c|c|c|c|c|c|}
\hline Author (year) & Region & City-State or State & $\mathrm{n}$ & Sample & $\begin{array}{l}\text { Questionnaire } \\
\text { used }\end{array}$ & $\begin{array}{l}\text { Quality } \\
\text { Indicator? }\end{array}$ & $\mathrm{SB}$ indicator & $\begin{array}{l}\text { Time } \\
\text { reference }\end{array}$ & $\begin{array}{l}\text { Cut-off } \\
\text { point }\end{array}$ \\
\hline $\begin{array}{l}\text { Lourenço et al. } \\
(2016)^{53}\end{array}$ & Northeast & Bahia State & 1243 & $\begin{array}{l}\text { College students } \\
17-54 \mathrm{y}\end{array}$ & ISAQ-A & Yes & Screen time & $\begin{array}{l}\text { Typical } \\
\text { weekday }\end{array}$ & $\geq 2 \mathrm{~h} /$ day \\
\hline $\begin{array}{l}\text { Gonçalves et al. } \\
(2019)^{54}\end{array}$ & Northeast & $\begin{array}{l}\text { Caruaru, } \\
\text { Pernambuco }\end{array}$ & 318 & $\begin{array}{l}\text { Parents and } \\
\text { their respective } \\
\text { children } 3-5 y\end{array}$ & $\begin{array}{l}\text { Instrument } \\
\text { developed by } \\
\text { He et al. }\end{array}$ & Yes & Screen time & $\begin{array}{l}\text { Typical } \\
\text { weekday and } \\
\text { weekend day }\end{array}$ & $\geq 3 \mathrm{~h} /$ day \\
\hline $\begin{array}{l}\text { Silva et al. } \\
(2017)^{55}\end{array}$ & Northeast & Ibicuí, Bahia & 310 & Elderly $\geq 60 y$ & IPAQ & No & Sitting time & $\begin{array}{l}\text { Typical } \\
\text { weekday and } \\
\text { weekend day }\end{array}$ & $\begin{array}{l}>414 \mathrm{~min} / \\
\text { day }\end{array}$ \\
\hline $\begin{array}{l}\text { Toscano et al. } \\
(2016)^{56}\end{array}$ & Northeast & Maceió, Alagoas & 156 & $\begin{array}{l}\text { Public Servers } \pm \\
39,8 \text { y }\end{array}$ & $\mathrm{x}$ & Yes & $\begin{array}{l}\text { Sitting time } \\
\text { and TV } \\
\text { watching }\end{array}$ & $\mathrm{x}$ & $\begin{array}{l}\mathrm{TV}>2 \mathrm{~h} / \text { day; } \\
\text { Sitting at } \\
\text { work } \geq 5,6 \mathrm{~h} / \\
\text { day }\end{array}$ \\
\hline $\begin{array}{l}\text { Tassitano et al. } \\
(2015)^{57}\end{array}$ & Northeast & Northeast region & 141309 & Adults $\geq 18 y$ & VIGITEL & No & TV watching & Typical day & $\geq 3 \mathrm{~h} /$ day \\
\hline $\begin{array}{l}\text { Farah et al. } \\
(2013)^{58}\end{array}$ & Northeast & Pernambuco State & 1910 & $\begin{array}{l}\text { Industry } \\
\text { workers > } 18 \mathrm{y}\end{array}$ & $\begin{array}{l}\text { "Estilo de } \\
\text { Vida e Hábitos } \\
\text { de Lazer dos } \\
\text { Trabalhadores } \\
\text { da Indústria" } \\
\text { Questionnaire }\end{array}$ & Yes & TV watching & $\begin{array}{l}\text { Weekdays } \\
\text { and weekend } \\
\text { days }\end{array}$ & $>2 \mathrm{~h} /$ day \\
\hline $\begin{array}{l}\text { Mussi et al. } \\
(2017)^{59}\end{array}$ & Northeast & Salvador, Bahia & 137 & $\begin{array}{l}\text { Women nursing } \\
\text { students } \geq 18 \mathrm{y}\end{array}$ & $\mathrm{x}$ & No & Sitting time & $\begin{array}{l}\text { Typical } \\
\text { weekday and } \\
\text { weekend day }\end{array}$ & $\geq 8 \mathrm{~h} /$ day \\
\hline $\begin{array}{l}\text { Marchesan et al. } \\
(2017)^{60}\end{array}$ & South & $\begin{array}{l}\text { Cruz Alta, Rio } \\
\text { Grande do Sul }\end{array}$ & 18 & $\begin{array}{l}\text { Patients on } \\
\text { hemodialysis } \\
>18 \mathrm{y}\end{array}$ & $\mathrm{x}$ & No & Sitting time & $\mathrm{x}$ & $\mathrm{x}$ \\
\hline $\begin{array}{l}\text { Gonçalves et al. } \\
(2017)^{61}\end{array}$ & South & Curitiba, Paraná & 1411 & Adults $18-65$ y & IPAQ & Yes & Sitting time & $\begin{array}{l}\text { Typical } \\
\text { weekday and } \\
\text { weekend day }\end{array}$ & $\geq 4 \mathrm{~h} /$ day \\
\hline $\begin{array}{l}\text { Felden et al. } \\
(2015)^{62}\end{array}$ & South & $\begin{array}{l}\text { Florianópolis, } \\
\text { Santa Catarina }\end{array}$ & 239 & $\begin{array}{l}\text { College students } \\
\pm 20,9 \mathrm{y}\end{array}$ & IPAQ & Yes & Sitting time & $\begin{array}{l}\text { Typical } \\
\text { weekday }\end{array}$ & $\begin{array}{l}>480 \mathrm{~min} / \\
\text { sem }\end{array}$ \\
\hline $\begin{array}{l}\text { Fronza et al. } \\
(2017)^{63}\end{array}$ & South & $\begin{array}{l}\text { Florianópolis, } \\
\text { Santa Catarina }\end{array}$ & 623 & $\begin{array}{l}\text { Public Servers } \\
20-69 y\end{array}$ & $\mathrm{x}$ & No & $\begin{array}{l}\text { Sitting time } \\
\text { and screen time }\end{array}$ & $\mathrm{x}$ & $\geq 3 \mathrm{~h} /$ day \\
\hline $\begin{array}{l}\text { Gutierre Filho } \\
\text { et al. }(2014)^{64}\end{array}$ & South & $\begin{array}{l}\text { Florianópolis, } \\
\text { Santa Catarina }\end{array}$ & 55 & $\begin{array}{l}\text { Elderly with } \\
\text { disabilities } \geq \\
60 \mathrm{y}\end{array}$ & IPAQ & Yes & Sitting time & $\mathrm{x}$ & $\mathrm{x}$ \\
\hline $\begin{array}{l}\text { Coledam } \\
(2019)^{65}\end{array}$ & South & Londrina, Paraná & 534 & Adults & $\mathrm{x}$ & Yes & Screen time & Typical day & $\geq 2 \mathrm{~h} /$ day \\
\hline $\begin{array}{l}\text { Souza et al. } \\
(2017)^{66}\end{array}$ & South & Londrina, Paraná & 959 & School teachers & $\mathrm{x}$ & No & $\mathrm{TV}$ watching & $\begin{array}{l}\text { Workdays } \\
\text { and } \\
\text { weekends }\end{array}$ & $\geq 2 \mathrm{~h} /$ day \\
\hline $\begin{array}{l}\text { Oliveira et al. } \\
(2020)^{67}\end{array}$ & South & Maringá, Paraná & 79 & Elderly & IPAQ & No & Sitting time & $\begin{array}{l}\text { Weekdays } \\
\text { and weekend } \\
\text { days }\end{array}$ & $\mathrm{x}$ \\
\hline $\begin{array}{l}\text { Ribeiro et al. } \\
(2018)^{68}\end{array}$ & South & Paraná State & 820 & Elderly $\geq 60 y$ & IPAQ & Yes & Sitting time & $\begin{array}{l}\text { Typical } \\
\text { weekday and } \\
\text { weekend day }\end{array}$ & $>4 \mathrm{~h} /$ day \\
\hline $\begin{array}{l}\text { Mielke et al. } \\
(2014)^{69}\end{array}$ & South & $\begin{array}{l}\text { Pelotas, Rio } \\
\text { Grande do Sul }\end{array}$ & 2927 & Adults $\geq 20 y$ & $\mathrm{x}$ & Yes & $\begin{array}{l}\text { Sitting time } \\
\text { and screen } \\
\text { time }\end{array}$ & $\begin{array}{l}\text { Typical } \\
\text { weekday }\end{array}$ & $>4,5 \mathrm{~h} / \mathrm{day}$ \\
\hline $\begin{array}{l}\text { Bueno et al. } \\
(2017)^{70}\end{array}$ & South & $\begin{array}{l}\text { Porto Alegre, Rio } \\
\text { Grande do Sul }\end{array}$ & 34 & $\begin{array}{l}\text { Patients on } \\
\text { hemodialysis } \\
>18 \mathrm{y}\end{array}$ & IPAQ & No & Sitting time & $\begin{array}{l}\text { Typical } \\
\text { weekday and } \\
\text { weekend day }\end{array}$ & $\mathrm{x}$ \\
\hline $\begin{array}{l}\text { Leão et al. } \\
(2020)^{71}\end{array}$ & South & $\begin{array}{l}\text { Rio Grande, Rio } \\
\text { Grande do Sul }\end{array}$ & 1131 & Elderly & $\begin{array}{l}\text { Measure of } \\
\text { Older Adult's } \\
\text { Sedentary Time } \\
\text { Questionnaire }\end{array}$ & Yes & $\begin{array}{l}\text { Sitting time } \\
\text { and TV } \\
\text { watching }\end{array}$ & Last week & $\begin{array}{l}\text { Sitting time: } \\
\text { 8h/day; TV } \\
\text { time: } 5 \mathrm{~h} / \\
\text { day }\end{array}$ \\
\hline
\end{tabular}

Continue... 
Continuation of Supplementary Table 2 - Main characteristics of studies that assessed sedentary behavior using subjective methods in adults or elderly.

\begin{tabular}{|c|c|c|c|c|c|c|c|c|c|}
\hline Author (year) & Region & City-State or State & $\mathrm{n}$ & Sample & $\begin{array}{l}\text { Questionnaire } \\
\text { used }\end{array}$ & $\begin{array}{l}\text { Quality } \\
\text { Indicator? }\end{array}$ & SB indicator & $\begin{array}{l}\text { Time } \\
\text { reference }\end{array}$ & $\begin{array}{l}\text { Cut-off } \\
\text { point }\end{array}$ \\
\hline $\begin{array}{l}\text { Cafruni et al. } \\
(2019)^{72}\end{array}$ & South & $\begin{array}{l}\text { São Leopoldo, Rio } \\
\text { Grande do Sul }\end{array}$ & 1079 & $\begin{array}{l}\text { Women } 20- \\
69 y\end{array}$ & $\mathrm{x}$ & Yes & Sitting time & $\begin{array}{l}\text { Any day of } \\
\text { the week and } \\
\text { weekend }\end{array}$ & $\begin{array}{l}\text { Transport- } \\
\text { related: } 17 \\
\text { min/day; } \\
\text { leisure time: } \\
163 \mathrm{~min} / \text { day }\end{array}$ \\
\hline $\begin{array}{l}\text { Rech et al. } \\
(2015)^{73}\end{array}$ & South & South region & 41156 & Adults $\geq 18 \mathrm{y}$ & VIGITEL & No & TV watching & Typical day & $\geq 3 \mathrm{~h} /$ day \\
\hline $\begin{array}{l}\text { Leite et al. } \\
(2019)^{74}\end{array}$ & Southeast & $\begin{array}{l}\text { Baependi, Minas } \\
\text { Gerais }\end{array}$ & 2027 & Adults $\geq 18 y$ & IPAQ & Yes & Sitting time & $\begin{array}{l}\text { Typical } \\
\text { weekday and } \\
\text { weekend day }\end{array}$ & $\mathrm{x}$ \\
\hline $\begin{array}{l}\text { Genebra et al. } \\
(2017)^{75}\end{array}$ & Southeast & Bauru, São Paulo & 600 & Adults $>20 y$ & $\mathrm{x}$ & No & Screen time & $\begin{array}{l}\text { Typical week } \\
\text { and typical } \\
\text { day }\end{array}$ & $>$ 3h/day \\
\hline $\begin{array}{l}\text { Turi et al. } \\
(2018)^{76}\end{array}$ & Southeast & Bauru, São Paulo & 970 & Adults $\geq 50 y$ & $\begin{array}{l}\text { Baecke } \\
\text { Questionnaire }\end{array}$ & Yes & TV watching & $\mathrm{x}$ & $\begin{array}{l}\text { Often and } \\
\text { very often }\end{array}$ \\
\hline $\begin{array}{l}\text { Machado et al. } \\
(2018)^{77}\end{array}$ & Southeast & $\begin{array}{l}\text { Belo Horizonte, } \\
\text { Minas Gerais }\end{array}$ & 378 & $\begin{array}{l}\text { Community- } \\
\text { dwelling } \geq 65 \mathrm{y}\end{array}$ & $\mathrm{x}$ & No & Sitting time & $\mathrm{x}$ & $\mathrm{x}$ \\
\hline $\begin{array}{l}\text { Moreira et al. } \\
(2017)^{78}\end{array}$ & Southeast & $\begin{array}{l}\text { Belo Horizonte, } \\
\text { Minas Gerais }\end{array}$ & 305 & Adults & VIGITEL & Yes & TV watching & $\mathrm{x}$ & $\geq 3 \mathrm{~h} /$ day \\
\hline $\begin{array}{l}\text { Duarte et al. } \\
(2013)^{79}\end{array}$ & Southeast & $\begin{array}{l}\text { Belo Horizonte, } \\
\text { Minas Gerais }\end{array}$ & 2016 & Adults $\geq 18 y$ & VIGITEL & No & TV watching & Typical day & $\geq 3 \mathrm{~h} /$ day \\
\hline $\begin{array}{l}\text { Carvalho et al. } \\
(2010)^{80}\end{array}$ & Southeast & $\begin{array}{l}\text { Campinas, São } \\
\text { Paulo }\end{array}$ & 271 & Elderly women & IPAQ & Yes & Sitting time & Last week & $\mathrm{x}$ \\
\hline $\begin{array}{l}\text { Atalla et al. } \\
(2019)^{81}\end{array}$ & Southeast & $\begin{array}{l}\text { Jaguariúna, São } \\
\text { Paulo }\end{array}$ & 1424 & Adults > $18 \mathrm{y}$ & VIGITEL & Yes & TV watching & $\mathrm{x}$ & $>$ 3h/day \\
\hline $\begin{array}{l}\text { Meneguci et al. } \\
(2015)^{82}\end{array}$ & Southeast & $\begin{array}{l}\text { Minas Gerais } \\
\text { State }\end{array}$ & 3296 & Elderly $\geq 60 y$ & IPAQ & Yes & Sitting time & $\begin{array}{l}\text { Typical } \\
\text { weekday and } \\
\text { weekend day }\end{array}$ & $\mathrm{x}$ \\
\hline $\begin{array}{l}\text { Ribeiro et al. } \\
(2018)^{83}\end{array}$ & Southeast & $\begin{array}{l}\text { Presidente } \\
\text { Prudente, São } \\
\text { Paulo }\end{array}$ & 102 & $\begin{array}{l}\text { Women breast } \\
\text { cancer survivors }\end{array}$ & $\mathrm{x}$ & No & Screen time & $\begin{array}{l}\text { Typical } \\
\text { weekday and } \\
\text { weekend day }\end{array}$ & $\geq 8 \mathrm{~h} /$ day \\
\hline $\begin{array}{l}\text { Oliveira et al. } \\
(2018)^{84}\end{array}$ & Southeast & $\begin{array}{l}\text { Presidente } \\
\text { Prudente, São } \\
\text { Paulo }\end{array}$ & 160 & $\begin{array}{l}\text { Patients with } \\
\text { nonspecific low } \\
\text { back pain } 18 \\
-60 \text { y }\end{array}$ & $\mathrm{x}$ & No & $\begin{array}{l}\text { General } \\
\text { indicator of } \\
\mathrm{SB}\end{array}$ & $\begin{array}{l}\text { Typical } \\
\text { weekday }\end{array}$ & $\mathrm{x}$ \\
\hline $\begin{array}{l}\text { Fernandes et al. } \\
(2010)^{85}\end{array}$ & Southeast & $\begin{array}{l}\text { Presidente } \\
\text { Prudente, São } \\
\text { Paulo }\end{array}$ & 1986 & Adults $\geq 30 y$ & $\begin{array}{l}\text { Baecke } \\
\text { Questionnaire }\end{array}$ & Yes & TV watching & $\mathrm{x}$ & $\begin{array}{l}\text { High } \\
\text { frequency }\end{array}$ \\
\hline $\begin{array}{l}\text { Fernandes et al. } \\
(2019)^{86}\end{array}$ & Southeast & $\begin{array}{l}\text { Presidente } \\
\text { Prudente, São } \\
\text { Paulo }\end{array}$ & 118 & Adults $40-65$ y & $\mathrm{x}$ & No & $\begin{array}{l}\text { Sitting time at } \\
\text { work }\end{array}$ & $\mathrm{x}$ & $\mathrm{x}$ \\
\hline $\begin{array}{l}\text { Bertolini et al. } \\
(2016)^{87}\end{array}$ & Southeast & $\begin{array}{l}\text { Presidente } \\
\text { Prudente, São } \\
\text { Paulo }\end{array}$ & 375 & Elderly $\geq 60 y$ & $\begin{array}{l}\text { Baecke } \\
\text { Questionnaire }\end{array}$ & Yes & TV watching & $\mathrm{x}$ & $\begin{array}{l}\text { Always } \\
\text { watching } \\
\text { TV }\end{array}$ \\
\hline $\begin{array}{l}\text { Moraes et al. } \\
(2013)^{88}\end{array}$ & Southeast & $\begin{array}{l}\text { Ribeirão Preto, } \\
\text { São Paulo }\end{array}$ & 1133 & Adults $\geq 30 y$ & IPAQ & Yes & Sitting time & $\begin{array}{l}\text { Typical } \\
\text { weekday and } \\
\text { weekend day }\end{array}$ & $\begin{array}{l}>308,6 \\
\min / \text { day }\end{array}$ \\
\hline $\begin{array}{l}\text { Suzuki, Moraes } \\
\text { and Freitas } \\
(2010)^{89}\end{array}$ & Southeast & $\begin{array}{l}\text { Ribeirão Preto, } \\
\text { São Paulo }\end{array}$ & 2197 & Adults $\geq 30 \mathrm{y}$ & IPAQ & Yes & Sitting time & Typical week & $\mathrm{x}$ \\
\hline $\begin{array}{l}\text { Gomes, Siqueira } \\
\text { and Sichieri } \\
(2001)^{90}\end{array}$ & Southeast & $\begin{array}{l}\text { Rio de Janeiro, Rio } \\
\text { de Janeiro }\end{array}$ & 4331 & $\begin{array}{l}\text { Adolescents and } \\
\text { adults } \geq 12 \mathrm{y}\end{array}$ & $\mathrm{x}$ & No & Screen time & $\mathrm{x}$ & $\geq 5 \mathrm{~h} /$ day \\
\hline $\begin{array}{l}\text { Monfort-Pires } \\
\text { et al. }(2014)^{91}\end{array}$ & Southeast & São Paulo State & 193 & $\begin{array}{l}\text { Pre-diabetics } \\
\text { Adults } 18-79 \text { y }\end{array}$ & IPAQ & Yes & TV watching & $\begin{array}{l}\text { Typical } \\
\text { weekday and } \\
\text { weekend day }\end{array}$ & $>3 \mathrm{~h} /$ day \\
\hline
\end{tabular}

Continue... 
Continuation of Supplementary Table 2 - Main characteristics of studies that assessed sedentary behavior using subjective methods in adults or elderly.

\begin{tabular}{|c|c|c|c|c|c|c|c|c|c|}
\hline Author (year) & Region & City-State or State & $\mathrm{n}$ & Sample & $\begin{array}{l}\text { Questionnaire } \\
\text { used }\end{array}$ & $\begin{array}{l}\text { Quality } \\
\text { Indicator? }\end{array}$ & $\mathrm{SB}$ indicator & $\begin{array}{l}\text { Time } \\
\text { reference }\end{array}$ & $\begin{array}{l}\text { Cut-off } \\
\text { point }\end{array}$ \\
\hline $\begin{array}{l}\text { Monteiro et al. } \\
(2008)^{92}\end{array}$ & Southeast & $\begin{array}{l}\text { São Paulo, São } \\
\text { Paulo }\end{array}$ & 2024 & Adults $\geq 18 y$ & VIGITEL & Yes & TV watching & Typical day & $\geq 3 \mathrm{~h} /$ day \\
\hline $\begin{array}{l}\text { Rocha et al. } \\
(2019)^{93}\end{array}$ & Southeast & $\begin{array}{l}\text { São Paulo, São } \\
\text { Paulo }\end{array}$ & 2512 & Adults $20-65$ y & IPAQ & Yes & Sitting time & $\begin{array}{l}\text { Typical } \\
\text { weekday and } \\
\text { weekend day }\end{array}$ & $>2 \mathrm{~h} / \mathrm{day}$ \\
\hline $\begin{array}{l}\text { Santos et al. } \\
(2017)^{94}\end{array}$ & Southeast & $\begin{array}{l}\text { Uberaba, Minas } \\
\text { Gerais }\end{array}$ & 622 & $\begin{array}{l}\text { Elderly } 60 \text { - } \\
96 \text { y }\end{array}$ & IPAQ & Yes & Sitting time & $\begin{array}{l}\text { Typical } \\
\text { weekday and } \\
\text { weekend day }\end{array}$ & $\begin{array}{l}75 \text { th } \\
\text { percentile }\end{array}$ \\
\hline $\begin{array}{l}\text { Silva et al. } \\
(2020)^{95}\end{array}$ & Southeast & $\begin{array}{l}\text { Uberaba, Minas } \\
\text { Gerais }\end{array}$ & 374 & Elderly $\geq 60 y$ & IPAQ & Yes & Sitting time & $\begin{array}{l}\text { Weekdays } \\
\text { and weekend } \\
\text { days }\end{array}$ & $\geq 7 \mathrm{~h} /$ day \\
\hline $\begin{array}{l}\text { Ferreira et al. } \\
(2019)^{96}\end{array}$ & Southeast & $\begin{array}{l}\text { Viçosa, Minas } \\
\text { Gerais }\end{array}$ & 854 & Adults $20-59 y$ & IPAQ & No & Sitting time & Weekdays & $>5 \mathrm{~h} / \mathrm{day}$ \\
\hline $\begin{array}{l}\text { Martinho et al. } \\
(2013)^{97}\end{array}$ & Southeast & $\begin{array}{l}\text { Viçosa, Minas } \\
\text { Gerais }\end{array}$ & 402 & Elderly $\geq 60 y$ & IPAQ & Yes & Sitting time & $\begin{array}{l}\text { Typical } \\
\text { weekday and } \\
\text { weekend day }\end{array}$ & $>2 \mathrm{~h} /$ day \\
\hline
\end{tabular}

Note: $\mathrm{y}$ = years; IPAQ = International Physical Activity Questionnaire ; PNS = "Pesquisa Nacional de Saúde"; VIGITEL = "Vigilância de fatores de risco e proteção para doenças crônicas por inquérito telefônico"; PNAD = "Pesquisa Nacional por Amostra de Domicílios"; ISAQ-A = Health Indicators and Quality of Life in Academics; GPAQ = Global Physical Activity Questionnaire; SB = sedentary behavior; TV = television; $\mathrm{h}=$ hours. 
Supplementary Table 3 - Main characteristics of studies that assessed sedentary behavior using subjective methods in children or adolescents.

\begin{tabular}{|c|c|c|c|c|c|c|c|c|c|}
\hline Author (year) & Region & $\begin{array}{l}\text { City-State or } \\
\text { State }\end{array}$ & $\mathrm{n}$ & Sample & $\begin{array}{l}\text { Questionnaire } \\
\text { used }\end{array}$ & $\begin{array}{l}\text { Quality } \\
\text { indicator? }\end{array}$ & SB indicator & $\begin{array}{l}\text { Time } \\
\text { reference }\end{array}$ & $\begin{array}{l}\text { Cut-off } \\
\text { point }\end{array}$ \\
\hline $\begin{array}{l}\text { Costa et al. } \\
(2018)^{98}\end{array}$ & Brazil & Brazil & 102072 & Students 9 th grade & $\begin{array}{l}\text { GSHS (version } \\
\text { used in PeNSE) }\end{array}$ & No & $\begin{array}{l}\text { Sitting time } \\
\text { and screen } \\
\text { time }\end{array}$ & $\begin{array}{l}\text { Typical } \\
\text { weekday }\end{array}$ & $>2 \mathrm{~h} /$ day \\
\hline $\begin{array}{l}\text { Oliveira et al. } \\
(2016)^{99}\end{array}$ & Brazil & Brazil & 74589 & Students $12-17 \mathrm{y}$ & $\mathrm{x}$ & No & Screen time & $\begin{array}{l}\text { Typical } \\
\text { weekday }\end{array}$ & $>2 \mathrm{~h} /$ day \\
\hline $\begin{array}{l}\text { Rezende et al. } \\
(2014)^{100}\end{array}$ & Brazil & Brazil & 109104 & Students 9 th grade & $\begin{array}{l}\text { GSHS (version } \\
\text { used in PeNSE) }\end{array}$ & No & $\begin{array}{l}\text { Sitting time } \\
\text { and screen } \\
\text { time }\end{array}$ & $\begin{array}{l}\text { Typical } \\
\text { weekday }\end{array}$ & $\geq 2 \mathrm{~h} /$ day \\
\hline $\begin{array}{l}\text { Hallal et al. } \\
(2010)^{101}\end{array}$ & Brazil & Brazil & 60973 & Students $13-15 \mathrm{y}$ & $\begin{array}{l}\text { GSHS (version } \\
\text { used in PeNSE) }\end{array}$ & Yes & $\begin{array}{l}\text { TV } \\
\text { watching }\end{array}$ & $\begin{array}{l}\text { Typical } \\
\text { weekday }\end{array}$ & $\geq 2 \mathrm{~h} /$ day \\
\hline $\begin{array}{l}\text { Nogueira } \\
\text { and Macedo } \\
(2009)^{102}\end{array}$ & Brazil & Brazil & 326 & $\begin{array}{l}\text { Physically active } \\
11-15 \mathrm{y}\end{array}$ & $\mathrm{x}$ & No & Screen time & $\begin{array}{l}\text { Weekdays and } \\
\text { weekend days }\end{array}$ & $\mathrm{x}$ \\
\hline $\begin{array}{l}\text { Machado et al. } \\
(2016)^{103}\end{array}$ & Brazil & Brazil & 1190 & $\begin{array}{l}\text { Male adolescents } \\
10-12 y\end{array}$ & $\begin{array}{l}\text { ENERGY Project } \\
\text { Questionnaire }\end{array}$ & No & Screen time & $\mathrm{x}$ & $>2 \mathrm{~h} /$ day \\
\hline $\begin{array}{l}\text { Silva et al. } \\
(2019)^{104}\end{array}$ & Brazil & $\begin{array}{l}\text { Porto Alegre, Rio } \\
\text { de Janeiro, Brasília } \\
\text { and Fortaleza }\end{array}$ & 1152 & Students $12-17 \mathrm{y}$ & $\mathrm{x}$ & No & Screen time & $\begin{array}{l}\text { Typica } \\
\text { weekday }\end{array}$ & $>2 \mathrm{~h} /$ day \\
\hline $\begin{array}{l}\text { Hardman et al. } \\
(2014)^{105}\end{array}$ & Brazil & $\begin{array}{l}\text { Recife, } \\
\text { Pernambuco and } \\
\text { Florianópolis, } \\
\text { Santa Catarina }\end{array}$ & 2155 & Students $15-24$ y & $\begin{array}{l}\text { "Saúde na boa" } \\
\text { Questionnaire }\end{array}$ & Yes & Screen time & $\begin{array}{l}\text { Typical } \\
\text { weekday and } \\
\text { weekend day }\end{array}$ & $\geq 2 \mathrm{~h} /$ day \\
\hline $\begin{array}{l}\text { Hackenhaar et } \\
\text { al. }(2013)^{106}\end{array}$ & Midwest & $\begin{array}{l}\text { Cuiabá, Mato } \\
\text { Grosso }\end{array}$ & 1716 & $\begin{array}{l}\text { Adolescents } 10 \\
-17 \mathrm{y}\end{array}$ & $\mathrm{x}$ & No & Screen time & $\begin{array}{l}\text { Typical } \\
\text { weekday }\end{array}$ & $\geq 4 \mathrm{~h} /$ day \\
\hline $\begin{array}{l}\text { Alexandre et al. } \\
(2016)^{107}\end{array}$ & Midwest & $\begin{array}{l}\text { Cuiabá, Mato } \\
\text { Grosso }\end{array}$ & 399 & $\begin{array}{l}\text { Adolescents } 12 \\
-19 y\end{array}$ & GSHS & Yes & $\begin{array}{l}\text { Sitting time } \\
\text { and screen } \\
\text { time }\end{array}$ & Typical day & $>2 \mathrm{~h} /$ day \\
\hline $\begin{array}{l}\text { Rauber et al. } \\
(2018)^{108}\end{array}$ & Midwest & Distrito Federal & 12 & $\begin{array}{l}\text { Overweight or } \\
\text { obese children } \\
9-11 \text { y }\end{array}$ & $\begin{array}{l}\text { Questionnaire } \\
\text { proposed by } \\
\text { Militão et al. }\end{array}$ & Yes & $\begin{array}{l}\text { General } \\
\text { indicator } \\
\text { of SB }\end{array}$ & Last week & $\mathrm{x}$ \\
\hline $\begin{array}{l}\text { Militão et al. } \\
(2013)^{109}\end{array}$ & Midwest & Distrito Federal & 112 & Students $10-13$ y & $\begin{array}{l}\text { "Avaliação do nível } \\
\text { de atividade física } \\
\text { e comportamento } \\
\text { sedentário para } \\
\text { adolescentes } \\
\text { com faixa etária } \\
\text { 10-13 anos" } \\
\text { Questionnaire }\end{array}$ & Yes & Screen time & Last week & $\mathrm{x}$ \\
\hline $\begin{array}{l}\text { Santos et al. } \\
(2018)^{110}\end{array}$ & Midwest & $\begin{array}{l}\text { Dourados, Mato } \\
\text { Grosso do Sul }\end{array}$ & 274 & Students $12-18$ y & $\mathrm{x}$ & No & Screen time & $\begin{array}{l}\text { Typical week } \\
\text { on weekdays } \\
\text { and on } \\
\text { weekends }\end{array}$ & $>2 \mathrm{~h} /$ day \\
\hline $\begin{array}{l}\text { Giugliano } \\
\text { and Carneiro } \\
(2004)^{111}\end{array}$ & Midwest & $\begin{array}{l}\text { Taguatinga, } \\
\text { Distrito Federal }\end{array}$ & 100 & Students $6-10 y$ & Sallis et al. & No & Sitting time & $\mathrm{x}$ & $\mathrm{x}$ \\
\hline $\begin{array}{l}\text { Gomes et al. } \\
(2020)^{112}\end{array}$ & North & $\begin{array}{l}\text { Manaus, } \\
\text { Amazonas }\end{array}$ & 376 & Students $12 \mathrm{y}$ & $\mathrm{x}$ & No & Screen time & $\begin{array}{l}\text { Typical } \\
\text { weekday }\end{array}$ & $\geq 4 \mathrm{~h} /$ day \\
\hline $\begin{array}{l}\text { Pinheiro et al. } \\
(2017)^{113}\end{array}$ & North & $\begin{array}{l}\text { Manaus, } \\
\text { Amazonas }\end{array}$ & 304 & Students $8-11 \mathrm{y}$ & $\begin{array}{l}\text { "Dia Típico de } \\
\text { Alimentação e } \\
\text { AF" }\end{array}$ & Yes & $\begin{array}{l}\text { Passive } \\
\text { transfer to } \\
\text { school }\end{array}$ & $\mathrm{x}$ & $\mathrm{x}$ \\
\hline $\begin{array}{l}\text { Bezerra et al. } \\
(2016)^{114}\end{array}$ & North & $\begin{array}{l}\text { Manaus, } \\
\text { Amazonas }\end{array}$ & 864 & Students $15-19 y$ & $\begin{array}{l}\text { "Lifestyle of } \\
\text { the Manauara } \\
\text { Adolescent" } \\
\text { Questionnaire }\end{array}$ & No & $\begin{array}{l}\text { Sitting time } \\
\text { and screen } \\
\text { time }\end{array}$ & $\mathrm{x}$ & $>2 \mathrm{~h} /$ day \\
\hline $\begin{array}{l}\text { Gordia et al. } \\
(2016)^{115}\end{array}$ & Northeast & Amargosa, Bahia & 1044 & Students $6-18$ y & PAQ-C & Yes & $\begin{array}{l}\text { TV } \\
\text { watching }\end{array}$ & Typical day & $\geq 3 \mathrm{~h} /$ day \\
\hline $\begin{array}{l}\text { Silva and } \\
\text { Santos Silva } \\
(2015)^{116}\end{array}$ & Northeast & Aracaju, Sergipe & 2243 & Students $13-18$ y & PAQ-C & No & Sitting time & Last week & $\mathrm{x}$ \\
\hline
\end{tabular}

Continue... 
Continuation of Supplementary Table 3 - Main characteristics of studies that assessed sedentary behavior using subjective methods in children or adolescents.

\begin{tabular}{|c|c|c|c|c|c|c|c|c|c|}
\hline Author (year) & Region & $\begin{array}{l}\text { City-State or } \\
\text { State }\end{array}$ & $\mathrm{n}$ & Sample & $\begin{array}{l}\text { Questionnaire } \\
\text { used }\end{array}$ & $\begin{array}{l}\text { Quality } \\
\text { indicator? }\end{array}$ & SB indicator & $\begin{array}{l}\text { Time } \\
\text { reference }\end{array}$ & $\begin{array}{l}\text { Cut-off } \\
\text { point }\end{array}$ \\
\hline $\begin{array}{l}\text { Silva et al. } \\
(2009)^{117}\end{array}$ & Northeast & Aracaju, Sergipe & 1028 & Students & PAQ-C & No & $\begin{array}{l}\text { TV } \\
\text { watching }\end{array}$ & Last week & $\mathrm{x}$ \\
\hline $\begin{array}{l}\text { Pitanga et al. } \\
(2016)^{118}\end{array}$ & Northeast & Camaçari, Bahia & 613 & $\begin{array}{l}\text { Adolescents } 15 \\
-18 \mathrm{y}\end{array}$ & $\mathrm{x}$ & No & Screen time & $\begin{array}{l}\text { Typical } \\
\text { weekday and } \\
\text { weekend day }\end{array}$ & $\geq 4 \mathrm{~h} / \mathrm{day}$ \\
\hline $\begin{array}{l}\text { Nunes, } \\
\text { Figueiroa and } \\
\text { Alves }(2007)^{119}\end{array}$ & Northeast & $\begin{array}{l}\text { Campina Grande, } \\
\text { Paraíba }\end{array}$ & 588 & Students $10-19 y$ & $\mathrm{x}$ & No & $\begin{array}{l}\text { TV } \\
\text { watching }\end{array}$ & $\mathrm{x}$ & $\geq 3 \mathrm{~h} /$ day \\
\hline $\begin{array}{l}\text { Costa et al. } \\
(2017)^{120}\end{array}$ & Northeast & $\begin{array}{l}\text { Campina Grande, } \\
\text { Paraíba }\end{array}$ & 576 & Students 15 - 19 y & $\mathrm{x}$ & No & Screen time & $\mathrm{x}$ & $\geq 2 \mathrm{~h} /$ day \\
\hline $\begin{array}{l}\text { Aros et al. } \\
(2017)^{121}\end{array}$ & Northeast & $\begin{array}{l}\text { Caruaru, } \\
\text { Pernambuco }\end{array}$ & $\begin{array}{l}600- \\
715\end{array}$ & Students $15-20 y$ & COMPAC & Yes & Screen time & $\begin{array}{l}\text { Weekdays and } \\
\text { weekend days }\end{array}$ & $\geq 3 \mathrm{~h} /$ day \\
\hline $\begin{array}{l}\text { Petribú et al. } \\
(2011)^{122}\end{array}$ & Northeast & $\begin{array}{l}\text { Caruaru, } \\
\text { Pernambuco }\end{array}$ & 600 & Students $15-20$ y & COMPAC & Yes & $\begin{array}{l}\text { TV } \\
\text { watching }\end{array}$ & $\mathrm{x}$ & $>3 \mathrm{~h} / \mathrm{day}$ \\
\hline $\begin{array}{l}\text { Martins et al. } \\
(2015)^{123}\end{array}$ & Northeast & Fortaleza, Ceará & 964 & Students $\geq 14 \mathrm{y}$ & $\mathrm{x}$ & No & Screen time & $\begin{array}{l}\text { Typical } \\
\text { weekday }\end{array}$ & $\geq 3 \mathrm{~h} /$ day \\
\hline $\begin{array}{l}\text { Bandeira et } \\
\text { al. }(2018)^{124}\end{array}$ & Northeast & Fortaleza, Ceará & 1085 & $\begin{array}{l}\text { Students } 7 \text { th }-9 \text { th } \\
\text { grade }\end{array}$ & YRBS & Yes & Screen time & $\mathrm{x}$ & $\geq 2 \mathrm{~h} /$ day \\
\hline $\begin{array}{l}\text { Munaro et al. } \\
(2016)^{125}\end{array}$ & Northeast & Jequié, Bahia & 1163 & Students $14-20 y$ & COMPAC & Yes & Screen time & $\mathrm{x}$ & $\geq 2 \mathrm{~h} /$ day \\
\hline $\begin{array}{l}\text { Guedes et al. } \\
(2012)^{126}\end{array}$ & Northeast & $\begin{array}{l}\text { João Pessoa, } \\
\text { Paraíba }\end{array}$ & 1268 & Students 15 - 18 y & IPAQ & No & Sitting time & $\begin{array}{l}\text { Typical } \\
\text { weekday and } \\
\text { weekend day }\end{array}$ & $\mathrm{x}$ \\
\hline $\begin{array}{l}\text { Farias Júnior et } \\
\text { al. }(2012)^{127}\end{array}$ & Northeast & $\begin{array}{l}\text { João Pessoa, } \\
\text { Paraíba }\end{array}$ & 2874 & Students $14-19 y$ & $\mathrm{x}$ & No & Screen time & $\begin{array}{l}\text { Weekdays and } \\
\text { weekend days }\end{array}$ & $>2 \mathrm{~h} /$ day \\
\hline $\begin{array}{l}\text { Silva, Lopes } \\
\text { and Silva } \\
(2007)^{128}\end{array}$ & Northeast & $\begin{array}{l}\text { João Pessoa, } \\
\text { Paraíba }\end{array}$ & 1570 & Students $7-12 y$ & $\mathrm{x}$ & Yes & Screen time & $\mathrm{x}$ & $\mathrm{x}$ \\
\hline $\begin{array}{l}\text { Rivera et al. } \\
(2010)^{129}\end{array}$ & Northeast & Maceió, Alogoas & 1253 & Students 7 - 17 y & PAQ-C & Yes & $\begin{array}{l}\text { TV } \\
\text { watching }\end{array}$ & $\mathrm{x}$ & $\geq 3 \mathrm{~h} /$ day \\
\hline $\begin{array}{l}\text { Siqueira, Alves } \\
\text { and Figueiroa } \\
(2009)^{130}\end{array}$ & Northeast & $\begin{array}{l}\text { Olinda, } \\
\text { Pernambuco }\end{array}$ & 86 & Children 5-9y & PAQ-C & No & $\begin{array}{l}\text { TV } \\
\text { watching }\end{array}$ & Last week & $\geq 3 \mathrm{~h} /$ day \\
\hline $\begin{array}{l}\text { Santos et al. } \\
(2017)^{131}\end{array}$ & Northeast & $\begin{array}{l}\text { Olinda, } \\
\text { Pernambuco }\end{array}$ & 253 & $\begin{array}{l}\text { Preschoolers } 24 \text { - } \\
59 \text { months }\end{array}$ & $\mathrm{x}$ & No & $\begin{array}{l}\text { TV } \\
\text { watching }\end{array}$ & $\mathrm{x}$ & $>2 \mathrm{~h} /$ day \\
\hline $\begin{array}{l}\text { Oliveira et al. } \\
(2018)^{132}\end{array}$ & Northeast & Pernambuco State & 6264 & Students $14-19 y$ & GSHS & Yes & $\begin{array}{l}\text { Sitting time } \\
\text { and screen } \\
\text { time }\end{array}$ & $\begin{array}{l}\text { Typical week } \\
\text { separately for } \\
\text { weekdays and } \\
\text { weekends }\end{array}$ & $>4 \mathrm{~h} / \mathrm{day}$ \\
\hline $\begin{array}{l}\text { Tassitano et al. } \\
(2009)^{133}\end{array}$ & Northeast & Pernambuco State & 4210 & Students $14-19 y$ & GSHS & Yes & $\begin{array}{l}\text { TV } \\
\text { watching }\end{array}$ & $\begin{array}{l}\text { Weekdays and } \\
\text { weekend days }\end{array}$ & $\geq 3 \mathrm{~h} /$ day \\
\hline $\begin{array}{l}\text { Lippo et al. } \\
(2010)^{134}\end{array}$ & Northeast & $\begin{array}{l}\text { Recife, } \\
\text { Pernambuco }\end{array}$ & 597 & Students $15-19 y$ & $\mathrm{x}$ & No & Screen time & $\begin{array}{l}\text { Typical } \\
\text { school day }\end{array}$ & $\geq 1 \mathrm{~h} /$ day \\
\hline $\begin{array}{l}\text { Oliveira et al. } \\
(2011)^{135}\end{array}$ & Northeast & $\begin{array}{l}\text { Recife, } \\
\text { Pernambuco }\end{array}$ & 65 & Students $3-6 y$ & $\mathrm{x}$ & Yes & $\begin{array}{l}\text { TV } \\
\text { watching }\end{array}$ & $\begin{array}{l}\text { Typical } \\
\text { weekday and } \\
\text { weekend day }\end{array}$ & $>2 \mathrm{~h} /$ day \\
\hline $\begin{array}{l}\text { Barbosa et al. } \\
(2019)^{136}\end{array}$ & Northeast & $\begin{array}{l}\text { Recife, } \\
\text { Pernambuco }\end{array}$ & 225 & $\begin{array}{l}\text { Adolescents } 10 \\
-19 y\end{array}$ & $\mathrm{x}$ & No & Screen time & $\mathrm{x}$ & $>2 \mathrm{~h} / \mathrm{day}$ \\
\hline $\begin{array}{l}\text { Brito Beck } \\
\text { da Silva et al. } \\
(2019)^{137}\end{array}$ & Northeast & Salvador, Bahia & 895 & $\begin{array}{l}\text { Students } 7 \text { th }-9 \text { th } \\
\text { grade }\end{array}$ & $\begin{array}{l}\text { GSHS (version } \\
\text { used in PeNSE) }\end{array}$ & Yes & Screen time & $\begin{array}{l}\text { Typical } \\
\text { weekday }\end{array}$ & $\geq 2 \mathrm{~h} /$ day \\
\hline $\begin{array}{l}\text { Alves et al. } \\
(2012)^{138}\end{array}$ & Northeast & Salvador, Bahia & 803 & Students $10-14$ y & $\mathrm{x}$ & No & Screen time & $\begin{array}{l}\text { Daily, weekly } \\
\text { and on } \\
\text { weekends }\end{array}$ & $\geq 3,3 \mathrm{~h} /$ day \\
\hline
\end{tabular}

Continue... 
Continuation of Supplementary Table 3 - Main characteristics of studies that assessed sedentary behavior using subjective methods in children or adolescents.

\begin{tabular}{|c|c|c|c|c|c|c|c|c|c|}
\hline Author (year) & Region & $\begin{array}{l}\text { City-State or } \\
\text { State }\end{array}$ & $\mathrm{n}$ & Sample & $\begin{array}{l}\text { Questionnaire } \\
\text { used }\end{array}$ & $\begin{array}{l}\text { Quality } \\
\text { indicator? }\end{array}$ & SB indicator & $\begin{array}{l}\text { Time } \\
\text { reference }\end{array}$ & $\begin{array}{l}\text { Cut-off } \\
\text { point }\end{array}$ \\
\hline $\begin{array}{l}\text { Oliveira et al. } \\
(2010)^{139}\end{array}$ & Northeast & $\begin{array}{l}\text { São Luiz, } \\
\text { Maranhão }\end{array}$ & 592 & Students $9-16$ y & $\begin{array}{l}\text { "Inquérito de } \\
\text { Atividade Física } \\
\text { Recordatório } \\
\text { de } 24 \text { h" adapted } \\
\text { from Self } \\
\text { Administered } \\
\text { Physical Activity } \\
\text { Checklist }\end{array}$ & Yes & Screen time & $\mathrm{x}$ & $>3,5 \mathrm{~h} /$ day \\
\hline $\begin{array}{l}\text { Simões et al. } \\
(2020)^{140}\end{array}$ & Northeast & $\begin{array}{l}\text { São Luiz, } \\
\text { Maranhão }\end{array}$ & 2515 & $\begin{array}{l}\text { Adolescents } 18 \\
-19 \mathrm{y}\end{array}$ & $\mathrm{x}$ & No & Screen time & $\mathrm{x}$ & $\geq 5 \mathrm{~h} /$ days \\
\hline $\begin{array}{l}\text { Silva et al. } \\
(2014)^{141}\end{array}$ & Northeast & Sergipe State & 2259 & Students $13-18$ y & PAQ-C & Yes & $\begin{array}{l}\text { TV } \\
\text { watching }\end{array}$ & Last week & $>2 \mathrm{~h} /$ day \\
\hline $\begin{array}{l}\text { Menezes } \\
\text { and Duarte } \\
(2015)^{142}\end{array}$ & Northeast & Sergipe State & 3992 & Students $14-19 y$ & GSHS & Yes & $\begin{array}{l}\text { Sitting time } \\
\text { and screen } \\
\text { time }\end{array}$ & Typical day & $>2 \mathrm{~h} /$ day \\
\hline $\begin{array}{l}\text { Santos et al. } \\
(2019)^{143}\end{array}$ & Northeast & Sergipe State & 3617 & Students $14-19 y$ & GSHS & No & $\begin{array}{l}\text { General } \\
\text { indicator } \\
\text { of SB }\end{array}$ & Typical day & $\geq 2 \mathrm{~h} /$ day \\
\hline $\begin{array}{l}\text { Geremia et al. } \\
(2015)^{144}\end{array}$ & South & $\begin{array}{l}\text { Bento Gonçalves, } \\
\text { Rio Grande do } \\
\text { Sul }\end{array}$ & 590 & Students $9-18$ y & $\mathrm{x}$ & No & Screen time & $\mathrm{x}$ & $\mathrm{x}$ \\
\hline $\begin{array}{l}\text { Suñé et al. } \\
(2007)^{145}\end{array}$ & South & $\begin{array}{l}\text { Capão da Canoa, } \\
\text { Rio Grande do } \\
\text { Sul }\end{array}$ & 719 & Students $11-13 \mathrm{y}$ & $\mathrm{x}$ & No & Screen time & $\mathrm{x}$ & $4,5 \mathrm{~h} / \mathrm{day}$ \\
\hline $\begin{array}{l}\text { Vasques and } \\
\text { Lopes }(2009)^{146}\end{array}$ & South & $\begin{array}{l}\text { Caxias do Sul, Rio } \\
\text { Grande do Sul }\end{array}$ & 1675 & Students $11-17 \mathrm{y}$ & $\mathrm{x}$ & Yes & Screen time & Typical week & $>2 \mathrm{~h} /$ day \\
\hline $\begin{array}{l}\text { Silva et al. } \\
(2018)^{147}\end{array}$ & South & $\begin{array}{l}\text { Criciúma, Santa } \\
\text { Catarina }\end{array}$ & 583 & Students $11-17 \mathrm{y}$ & PAQ-C & Yes & Sitting time & Last week & $\begin{array}{l}\text { Remained } \\
\text { seating }\end{array}$ \\
\hline $\begin{array}{l}\text { Azambuja et al. } \\
(2012)^{148}\end{array}$ & South & $\begin{array}{l}\text { Cruzeiro do } \\
\text { Oeste, Paraná }\end{array}$ & 1074 & Students $6-10 y$ & $\mathrm{x}$ & No & $\begin{array}{l}\text { TV } \\
\text { watching }\end{array}$ & $\mathrm{x}$ & $>4 \mathrm{~h} /$ day \\
\hline $\begin{array}{l}\text { Ulbricht et al. } \\
(2018)^{149}\end{array}$ & South & Curitiba, Paraná & 675 & Students $11-18 \mathrm{y}$ & IPAQ & Yes & Sitting time & $\begin{array}{l}\text { Weekdays and } \\
\text { weekend days }\end{array}$ & $>2 \mathrm{~h} /$ day \\
\hline $\begin{array}{l}\text { Prado et al. } \\
(2017)^{150}\end{array}$ & South & Curitiba, Paraná & 1081 & Students $11-18 \mathrm{y}$ & $\mathrm{x}$ & No & Screen time & $\begin{array}{l}\text { Typical } \\
\text { weekday }\end{array}$ & $\geq 2 \mathrm{~h} /$ day \\
\hline $\begin{array}{l}\text { Alberico et al. } \\
(2017)^{151}\end{array}$ & South & Curitiba, Paraná & 381 & Students $12-17 \mathrm{y}$ & $\mathrm{x}$ & No & Sitting time & $\begin{array}{l}\text { Weekdays and } \\
\text { weekend days }\end{array}$ & $\begin{array}{l}\text { Over } 60 \% \\
\text { of this time }\end{array}$ \\
\hline $\begin{array}{l}\text { Machado- } \\
\text { Rodrigues et al. } \\
(2015)^{152}\end{array}$ & South & Curitiba, Paraná & 262 & $\begin{array}{l}\text { Female students } \\
14-17 \text { y }\end{array}$ & $\begin{array}{l}\text { Bouchard et } \\
\text { al.; Machado- } \\
\text { Rodrigues et al. }\end{array}$ & Yes & $\begin{array}{l}\text { TV } \\
\text { watching }\end{array}$ & $\mathrm{x}$ & $\mathrm{x}$ \\
\hline $\begin{array}{l}\text { Barbosa Filho } \\
\text { et al. }(2012)^{153}\end{array}$ & South & Curitiba, Paraná & 1628 & $\begin{array}{l}\text { Students } 6 \text { th }-2 \text { th } \\
\text { grade }\end{array}$ & YRBS & Yes & $\begin{array}{l}\text { TV } \\
\text { watching }\end{array}$ & $\begin{array}{l}\text { Weekdays and } \\
\text { weekend days }\end{array}$ & $\geq 3 \mathrm{~h} /$ day \\
\hline $\begin{array}{l}\text { Bacil et al. } \\
(2018)^{9}\end{array}$ & South & Curitiba, Paraná & 117 & Students $9-15 \mathrm{y}$ & ASAQ & Yes & $\begin{array}{l}\text { General } \\
\text { indicator } \\
\text { of SB }\end{array}$ & $\begin{array}{l}\text { typical week } \\
\text { and weekend }\end{array}$ & $\mathrm{x}$ \\
\hline $\begin{array}{l}\text { Guimarães et al. } \\
(2013)^{154}\end{array}$ & South & Curitiba, Paraná & 572 & Students $12-17 \mathrm{y}$ & ASAQ & Yes & $\begin{array}{l}\text { General } \\
\text { indicator } \\
\text { of SB }\end{array}$ & $\begin{array}{l}\text { Weekdays and } \\
\text { weekend days }\end{array}$ & $\mathrm{x}$ \\
\hline $\begin{array}{l}\text { Silva et al. } \\
(2019)^{155}\end{array}$ & South & Curitiba, Paraná & 893 & Students $11-17 \mathrm{y}$ & ASAQ & Yes & Screen time & $\mathrm{x}$ & $\mathrm{x}$ \\
\hline $\begin{array}{l}\text { Schwertner et } \\
\text { al. }(2020)^{156}\end{array}$ & South & $\begin{array}{l}\text { Florianópolis, } \\
\text { Santa Catarina }\end{array}$ & 330 & Students $15-18 \mathrm{y}$ & $\mathrm{x}$ & No & Screen time & Typical day & $\geq 2 \mathrm{~h} /$ day \\
\hline $\begin{array}{l}\text { Lobo et al. } \\
(2019)^{157}\end{array}$ & South & $\begin{array}{l}\text { Florianópolis, } \\
\text { Santa Catarina }\end{array}$ & 5364 & Students $7-12 y$ & Web-CAAFE & Yes & Screen time & $\begin{array}{l}\text { Previous day } \\
\text { (24-h recall) }\end{array}$ & $\begin{array}{l}\text { Third } \\
\text { tertile }\end{array}$ \\
\hline $\begin{array}{l}\text { Berria et al. } \\
(2018)^{158}\end{array}$ & South & $\begin{array}{l}\text { Florianópolis, } \\
\text { Santa Catarina }\end{array}$ & 210 & $\begin{array}{l}\text { Students } 6 \text { th - } \\
\text { 9th grade }\end{array}$ & $\mathrm{x}$ & No & Screen time & $\begin{array}{l}\text { During the } \\
\text { week and on } \\
\text { weekends }\end{array}$ & $\mathrm{x}$ \\
\hline
\end{tabular}

Continue... 
Continuation of Supplementary Table 3 - Main characteristics of studies that assessed sedentary behavior using subjective methods in children or adolescents.

\begin{tabular}{|c|c|c|c|c|c|c|c|c|c|}
\hline Author (year) & Region & $\begin{array}{l}\text { City-State or } \\
\text { State }\end{array}$ & $\mathrm{n}$ & Sample & $\begin{array}{l}\text { Questionnaire } \\
\text { used }\end{array}$ & $\begin{array}{l}\text { Quality } \\
\text { indicator? }\end{array}$ & SB indicator & $\begin{array}{l}\text { Time } \\
\text { reference }\end{array}$ & $\begin{array}{l}\text { Cut-off } \\
\text { point }\end{array}$ \\
\hline $\begin{array}{l}\text { Pelegrini } \\
\text { and Petroski } \\
(2007)^{159}\end{array}$ & South & $\begin{array}{l}\text { Florianópolis, } \\
\text { Santa Catarina }\end{array}$ & 653 & Students $14-18 \mathrm{y}$ & $\mathrm{x}$ & No & Screen time & $\mathrm{x}$ & $>4 \mathrm{~h} / \mathrm{day}$ \\
\hline $\begin{array}{l}\text { Pinho et al. } \\
(2017)^{160}\end{array}$ & South & $\begin{array}{l}\text { Florianópolis, } \\
\text { Santa Catarina }\end{array}$ & 963 & Students $11-14$ y & $\mathrm{x}$ & No & Screen time & $\begin{array}{l}\text { Typical } \\
\text { weekday and } \\
\text { weekend day }\end{array}$ & $>2 \mathrm{~h} / \mathrm{day}$ \\
\hline $\begin{array}{l}\text { Christofoletti et } \\
\text { al. }(2016)^{161}\end{array}$ & South & $\begin{array}{l}\text { Florianópolis, } \\
\text { Santa Catarina }\end{array}$ & 1831 & Students $7-10 y$ & Web-CAAFE & Yes & $\begin{array}{l}\text { General } \\
\text { indicator } \\
\text { of SB }\end{array}$ & Last week & $1 \mathrm{SB}$ \\
\hline $\begin{array}{l}\text { Costa and Assis } \\
(2011)^{162}\end{array}$ & South & $\begin{array}{l}\text { Florianópolis, } \\
\text { Santa Catarina }\end{array}$ & 2936 & Students $7-10 y$ & $\mathrm{x}$ & No & Screen time & $\begin{array}{l}\text { Typical } \\
\text { weekday and } \\
\text { weekend day }\end{array}$ & $\geq 4 \mathrm{~h} / \mathrm{day}$ \\
\hline $\begin{array}{l}\text { Oliveira } \\
\text { and Guedes } \\
(2019)^{163}\end{array}$ & South & $\begin{array}{l}\text { Jacarezinho, } \\
\text { Paraná }\end{array}$ & 1035 & Students $12-20 \mathrm{y}$ & $\mathrm{x}$ & No & Screen time & $\begin{array}{l}\text { Typical week } \\
\text { and weekend }\end{array}$ & $>2 \mathrm{~h} /$ day \\
\hline $\begin{array}{l}\text { Silva et al. } \\
(2010)^{164}\end{array}$ & South & $\begin{array}{l}\text { Jacarezinho, } \\
\text { Paraná }\end{array}$ & 114 & Students $6-14 y$ & $\mathrm{x}$ & No & Screen time & $\begin{array}{l}\text { Specific days } \\
\text { on the last } \\
\text { week }\end{array}$ & $>2 \mathrm{~h} /$ day \\
\hline $\begin{array}{l}\text { Werneck et al. } \\
(2018)^{165}\end{array}$ & South & Londrina, Paraná & 1209 & $\begin{array}{l}\text { Students } 10-17 \mathrm{y} \\
\text { and parents }\end{array}$ & $\mathrm{x}$ & Yes & Screen time & $\begin{array}{l}\text { Typical } \\
\text { weekday and } \\
\text { weekend day }\end{array}$ & $\mathrm{x}$ \\
\hline $\begin{array}{l}\text { Greca et al. } \\
(2016)^{166}\end{array}$ & South & Londrina, Paraná & 480 & Students $8-17 y$ & $\mathrm{x}$ & No & Screen time & $\mathrm{x}$ & $\geq 2 \mathrm{~h} / \mathrm{day}$ \\
\hline $\begin{array}{l}\text { Silva et al. } \\
(2016)^{167}\end{array}$ & South & Londrina, Paraná & 1321 & Students $10-16$ y & $\mathrm{x}$ & Yes & $\begin{array}{l}\text { TV } \\
\text { watching }\end{array}$ & $\begin{array}{l}\text { Weekdays and } \\
\text { weekend days }\end{array}$ & $>4 \mathrm{~h} / \mathrm{day}$ \\
\hline $\begin{array}{l}\text { Christofaro et } \\
\text { al. }(2015)^{168}\end{array}$ & South & Londrina, Paraná & 1231 & Students $14-17 \mathrm{y}$ & $\mathrm{x}$ & No & Screen time & $\mathrm{x}$ & $\geq 2 \mathrm{~h} /$ day \\
\hline $\begin{array}{l}\text { Coledam et al. } \\
(2014)^{169}\end{array}$ & South & Londrina, Paraná & 738 & Students $10-17 \mathrm{y}$ & $\mathrm{x}$ & Yes & Screen time & Weekdays & $>2 \mathrm{~h} /$ day \\
\hline $\begin{array}{l}\text { Felden et al. } \\
(2016)^{170}\end{array}$ & South & $\begin{array}{l}\text { Maravilha, Santa } \\
\text { Catarina }\end{array}$ & 516 & Students $10-19 y$ & IPAQ & Yes & Sitting time & $\begin{array}{l}\text { Typical } \\
\text { weekday }\end{array}$ & $\mathrm{x}$ \\
\hline $\begin{array}{l}\text { Moraes et al. } \\
(2009)^{171}\end{array}$ & South & Maringá, Paraná & 991 & Students $14-18$ y & $\mathrm{x}$ & Yes & Screen time & $\mathrm{x}$ & $\geq 4 \mathrm{~h} /$ day \\
\hline $\begin{array}{l}\text { Guimarães et al. } \\
(2013)^{172}\end{array}$ & South & Paraná State & 122 & Students $12-17 \mathrm{y}$ & ASAQ & Yes & $\begin{array}{l}\text { General } \\
\text { indicator } \\
\text { of SB }\end{array}$ & $\begin{array}{l}\text { Weekdays and } \\
\text { weekend days }\end{array}$ & $\mathrm{x}$ \\
\hline $\begin{array}{l}\text { Oliveira et al. } \\
(2020)^{173}\end{array}$ & South & Paranavaí, Paraná & 2764 & Students $10-18 \mathrm{y}$ & $\mathrm{x}$ & Yes & Screen time & $\mathrm{x}$ & $>2 \mathrm{~h} /$ day \\
\hline $\begin{array}{l}\text { Ferreira et al. } \\
(2016)^{174}\end{array}$ & South & $\begin{array}{l}\text { Pelotas, Rio } \\
\text { Grande do Sul }\end{array}$ & 8661 & $\begin{array}{l}\text { Students } 5 \text { th - } \\
12 \text { th grade }\end{array}$ & $\begin{array}{l}\text { "HELENA" } \\
\text { instrument }\end{array}$ & Yes & $\begin{array}{l}\text { Sitting time } \\
\text { and screen } \\
\text { time }\end{array}$ & $\begin{array}{l}\text { Weekdays and } \\
\text { weekend days }\end{array}$ & $>2 \mathrm{~h} / \mathrm{day}$ \\
\hline $\begin{array}{l}\text { Hallal et al. } \\
(2006)^{175}\end{array}$ & South & $\begin{array}{l}\text { Pelotas, Rio } \\
\text { Grande do Sul }\end{array}$ & 4452 & Students $10-12$ y & $\mathrm{x}$ & No & Screen time & $\mathrm{x}$ & $\geq 1 \mathrm{~h} /$ day \\
\hline $\begin{array}{l}\text { Xavier et al. } \\
(2014)^{176}\end{array}$ & South & $\begin{array}{l}\text { Pelotas, Rio } \\
\text { Grande do Sul }\end{array}$ & 372 & Students $14-19 y$ & $\mathrm{x}$ & No & $\begin{array}{l}\text { TV } \\
\text { watching }\end{array}$ & $\mathrm{x}$ & $\geq 2 \mathrm{~h} /$ day \\
\hline $\begin{array}{l}\text { Bacil et al. } \\
(2013)^{177}\end{array}$ & South & $\begin{array}{l}\text { Ponta Grossa, } \\
\text { Paraná }\end{array}$ & 1129 & Students $14-18$ y & $\mathrm{x}$ & No & Screen time & $\mathrm{x}$ & $\geq 5 \mathrm{~h} /$ day \\
\hline $\begin{array}{l}\text { Lopes et al. } \\
(2014)^{178}\end{array}$ & South & $\begin{array}{l}\text { Santa Catarina } \\
\text { State }\end{array}$ & 6529 & Students 15 - 19 y & COMPAC & No & Screen time & $\begin{array}{l}\text { Weekdays and } \\
\text { weekend days }\end{array}$ & $\geq 4 \mathrm{~h} / \mathrm{day}$ \\
\hline $\begin{array}{l}\text { Corso et al. } \\
(2012)^{179}\end{array}$ & South & $\begin{array}{l}\text { Santa Catarina } \\
\text { State }\end{array}$ & 4964 & $\begin{array}{l}\text { Students } 1 \text { st }-4 \text { th } \\
\text { grade }\end{array}$ & $\mathrm{x}$ & No & Screen time & $\begin{array}{l}\text { Weekdays and } \\
\text { weekend days }\end{array}$ & $>2 \mathrm{~h} /$ day \\
\hline $\begin{array}{l}\text { Silva et al. } \\
(2008)^{180}\end{array}$ & South & $\begin{array}{l}\text { Santa Catarina } \\
\text { State }\end{array}$ & 5028 & Students $15-19 y$ & COMPAC & Yes & Screen time & Weekdays & $\geq 2 \mathrm{~h} /$ day \\
\hline $\begin{array}{l}\text { Spohr et al. } \\
(2012)^{181}\end{array}$ & South & $\begin{array}{l}\text { Santa Maria, Rio } \\
\text { Grande do Sul }\end{array}$ & 273 & $\begin{array}{l}\text { Students } 1 \text { st } \\
\text { elementar grade }\end{array}$ & $\mathrm{x}$ & No & Screen time & $\mathrm{x}$ & $\geq 5 \mathrm{~h} /$ day \\
\hline
\end{tabular}

Continue... 
Continuation of Supplementary Table 3 - Main characteristics of studies that assessed sedentary behavior using subjective methods in children or adolescents.

\begin{tabular}{|c|c|c|c|c|c|c|c|c|c|}
\hline Author (year) & Region & $\begin{array}{l}\text { City-State or } \\
\text { State }\end{array}$ & $\mathrm{n}$ & Sample & $\begin{array}{l}\text { Questionnaire } \\
\text { used }\end{array}$ & $\begin{array}{l}\text { Quality } \\
\text { indicator? }\end{array}$ & SB indicator & $\begin{array}{l}\text { Time } \\
\text { reference }\end{array}$ & $\begin{array}{l}\text { Cut-off } \\
\text { point }\end{array}$ \\
\hline $\begin{array}{l}\text { Faria et al. } \\
(2015)^{182}\end{array}$ & South & $\begin{array}{l}\text { Santo Antônio da } \\
\text { Platina, Paraná }\end{array}$ & 72 & Students $9-12 y$ & $\mathrm{x}$ & No & Screen time & $\begin{array}{l}\text { Typical } \\
\text { weekday and } \\
\text { weekend day }\end{array}$ & $\mathrm{x}$ \\
\hline $\begin{array}{l}\text { Piola et al. } \\
(2019)^{183}\end{array}$ & South & $\begin{array}{l}\text { São José dos } \\
\text { Pinhais, Paraná }\end{array}$ & 786 & $\begin{array}{l}\text { High school } \\
\text { students }\end{array}$ & ASAQ & Yes & Screen time & $\begin{array}{l}\text { Typical } \\
\text { weekday and } \\
\text { weekend day }\end{array}$ & $\geq 2 \mathrm{~h} /$ day \\
\hline $\begin{array}{l}\text { Gonçalves and } \\
\text { Silva }(2016)^{184}\end{array}$ & South & $\begin{array}{l}\text { São José, } \\
\text { Florianópolis, } \\
\text { Santa Catarina }\end{array}$ & 879 & Students $14-19 y$ & YRBS & Yes & Screen time & $\begin{array}{l}\text { Weekdays and } \\
\text { weekend days }\end{array}$ & $\geq 2 \mathrm{~h} /$ day \\
\hline $\begin{array}{l}\text { Weber et al. } \\
(2015)^{185}\end{array}$ & South & $\begin{array}{l}\text { São Leopoldo, Rio } \\
\text { Grande do Sul }\end{array}$ & 813 & Students 6 y & $\begin{array}{l}\text { National Health } \\
\text { and Nutrition } \\
\text { Examination } \\
\text { Survey } \\
\text { (NHANES) }\end{array}$ & Yes & Screen time & Typical day & $>2 \mathrm{~h} / \mathrm{day}$ \\
\hline $\begin{array}{l}\text { Beck et al. } \\
(2014)^{186}\end{array}$ & South & $\begin{array}{l}\text { Três de Maio, Rio } \\
\text { Grande do Sul }\end{array}$ & 660 & Students $14-19 y$ & $\mathrm{x}$ & No & Screen time & $\begin{array}{l}\text { Weekdays and } \\
\text { weekend days }\end{array}$ & $\mathrm{x}$ \\
\hline $\begin{array}{l}\text { Silva et al. } \\
(2015)^{187}\end{array}$ & South & $\begin{array}{l}\text { Uruguaiana, Rio } \\
\text { Grande do Sul }\end{array}$ & 1455 & Students $10-17 y$ & $\mathrm{x}$ & No & Screen time & $\mathrm{x}$ & $>3 \mathrm{~h} /$ day \\
\hline $\begin{array}{l}\text { Bordon et al. } \\
(2019)^{188}\end{array}$ & Southeast & $\begin{array}{l}\text { Americana, São } \\
\text { Paulo }\end{array}$ & 703 & Students $9-10 y$ & $\mathrm{x}$ & No & Screen time & $\mathrm{x}$ & $>2 \mathrm{~h} /$ day \\
\hline $\begin{array}{l}\text { Araujo et al. } \\
(2018)^{189}\end{array}$ & Southeast & Bauru, São Paulo & 270 & $\begin{array}{l}\text { Children and } \\
\text { adolescents } 7 \text { - } \\
12 \mathrm{y}\end{array}$ & PAQ-C & No & Screen time & Last week & $\geq 2 \mathrm{~h} /$ day \\
\hline $\begin{array}{l}\text { Vitta et al. } \\
(2011)^{190}\end{array}$ & Southeast & Bauru, São Paulo & 1236 & $\begin{array}{l}\text { Students } 5 \text { th }-8 \text { th } \\
\text { grade }\end{array}$ & $\mathrm{x}$ & No & Screen time & $\begin{array}{l}\text { Typical school } \\
\text { week and } \\
\text { school day }\end{array}$ & $\begin{array}{l}>2 \mathrm{~h} / \\
\text { day and } 2 \\
\text { times }\end{array}$ \\
\hline $\begin{array}{l}\text { Vitta et al. } \\
(2013)^{191}\end{array}$ & Southeast & Bauru, São Paulo & 524 & Students $10-14 \mathrm{y}$ & $\mathrm{x}$ & No & Screen time & Typical week & $\geq 3 \mathrm{~h} /$ day \\
\hline $\begin{array}{l}\text { Mondini et al. } \\
(2007)^{192}\end{array}$ & Southeast & $\begin{array}{l}\text { Cajamar, São } \\
\text { Paulo }\end{array}$ & 1014 & Students 1 st grade & $\mathrm{x}$ & No & $\begin{array}{l}\text { TV } \\
\text { watching }\end{array}$ & $\mathrm{x}$ & $\geq 4 \mathrm{~h} /$ day \\
\hline $\begin{array}{l}\text { Braz et al. } \\
(2019)^{193}\end{array}$ & Southeast & $\begin{array}{l}\text { Campinas, São } \\
\text { Paulo }\end{array}$ & 924 & $\begin{array}{l}\text { Adolescents } 10 \\
-19 y\end{array}$ & $\mathrm{x}$ & No & Screen time & $\mathrm{x}$ & $>3 \mathrm{~h} / \mathrm{day}$ \\
\hline $\begin{array}{l}\text { Andrade Neto } \\
\text { et al. }(2014)^{194}\end{array}$ & Southeast & $\begin{array}{l}\text { Espírito Santo } \\
\text { State }\end{array}$ & 1770 & Students $7-10 y$ & $\begin{array}{l}\text { Questionnaire for } \\
\text { children applied } \\
\text { in the SAUDES } \\
\text { study }\end{array}$ & Yes & Screen time & $\mathrm{x}$ & $>$ 2h/day \\
\hline $\begin{array}{l}\text { Souza et al. } \\
(2016)^{195}\end{array}$ & Southeast & $\begin{array}{l}\text { Guaxupé, Minas } \\
\text { Gerais }\end{array}$ & 91 & Students $\pm 9 y$ & $\mathrm{x}$ & No & $\begin{array}{l}\text { Sitting time } \\
\text { and TV } \\
\text { watching }\end{array}$ & $\mathrm{x}$ & $\mathrm{x}$ \\
\hline $\begin{array}{l}\text { Victo et al. } \\
(2017)^{196}\end{array}$ & Southeast & $\begin{array}{l}\text { Ilhabela, São } \\
\text { Paulo }\end{array}$ & 181 & $\begin{array}{l}\text { Adolescents } 11 \\
-18 \mathrm{y}\end{array}$ & $\begin{array}{l}\text { IPAQ and Diet } \\
\text { and Lifestyle } \\
\text { Questionnaire }\end{array}$ & Yes & $\begin{array}{l}\text { Sitting time } \\
\text { and TV } \\
\text { watching }\end{array}$ & $\begin{array}{l}\text { Typical } \\
\text { weekday and } \\
\text { weekend day } \\
\text { for sitting time } \\
\text { and typical } \\
\text { school day for } \\
\text { Tv watching }\end{array}$ & $\geq 2 \mathrm{~h} /$ day \\
\hline $\begin{array}{l}\text { Lima-Junior et } \\
\text { al. }(2019)^{22}\end{array}$ & Southeast & $\begin{array}{l}\text { Minas Gerais } \\
\text { State }\end{array}$ & 153 & Students $10-12 \mathrm{y}$ & $\mathrm{x}$ & No & $\begin{array}{l}\text { TV } \\
\text { watching }\end{array}$ & Typical day & $\mathrm{x}$ \\
\hline $\begin{array}{l}\text { Vasconcellos et } \\
\text { al. }(2013)^{197}\end{array}$ & Southeast & $\begin{array}{l}\text { Niterói, Rio de } \\
\text { Janeiro }\end{array}$ & 328 & Students $10-18 y$ & $\begin{array}{l}\text { Daily activity } \\
\text { semi-structured } \\
\text { questionnaire } \\
\text { adapted from } \\
\text { Barros and Nahas }\end{array}$ & No & Screen time & $\begin{array}{l}\text { Weekdays and } \\
\text { weekend days }\end{array}$ & $\mathrm{x}$ \\
\hline $\begin{array}{l}\text { Silva and } \\
\text { Malina } \\
(2000)^{198}\end{array}$ & Southeast & $\begin{array}{l}\text { Niterói, Rio de } \\
\text { Janeiro }\end{array}$ & 325 & Students $10-15 y$ & PAQ-C & Yes & $\begin{array}{l}\text { TV } \\
\text { watching }\end{array}$ & $\mathrm{x}$ & $\mathrm{x}$ \\
\hline
\end{tabular}

Continue... 
Continuation of Supplementary Table 3 - Main characteristics of studies that assessed sedentary behavior using subjective methods in children or adolescents.

\begin{tabular}{|c|c|c|c|c|c|c|c|c|c|}
\hline Author (year) & Region & $\begin{array}{l}\text { City-State or } \\
\text { State }\end{array}$ & $\mathrm{n}$ & Sample & $\begin{array}{l}\text { Questionnaire } \\
\text { used }\end{array}$ & $\begin{array}{l}\text { Quality } \\
\text { indicator? }\end{array}$ & SB indicator & $\begin{array}{l}\text { Time } \\
\text { reference }\end{array}$ & $\begin{array}{l}\text { Cut-off } \\
\text { point }\end{array}$ \\
\hline $\begin{array}{l}\text { Fonseca, } \\
\text { Sichieri and } \\
\text { Veiga }(1998)^{199}\end{array}$ & Southeast & $\begin{array}{l}\text { Niterói, Rio de } \\
\text { Janeiro }\end{array}$ & 391 & Students $15-17 \mathrm{y}$ & $\mathrm{x}$ & No & Screen time & $\mathrm{x}$ & $>3 \mathrm{~h} / \mathrm{day}$ \\
\hline $\begin{array}{l}\text { Rodrigues et al. } \\
(2020)^{200}\end{array}$ & Southeast & $\begin{array}{l}\text { Niterói, Rio de } \\
\text { Janeiro }\end{array}$ & 437 & Students $10-16 y$ & $\mathrm{x}$ & No & Screen time & typical day & $\geq 5 \mathrm{~h} /$ day \\
\hline $\begin{array}{l}\text { Fernandes et al. } \\
(2015)^{201}\end{array}$ & Southeast & $\begin{array}{l}\text { Ourinhos, São } \\
\text { Paulo }\end{array}$ & 1461 & Students $10-14$ y & $\mathrm{x}$ & No & Screen time & $\begin{array}{l}\text { Typical school } \\
\text { week and } \\
\text { school day }\end{array}$ & $\begin{array}{l}2 \mathrm{~h} / \mathrm{day} \\
\text { and } 2 \\
\text { times/ } \\
\text { week }\end{array}$ \\
\hline $\begin{array}{l}\text { Caixeta } \\
\text { and Amato } \\
(2020)^{202}\end{array}$ & Southeast & $\begin{array}{l}\text { Patos de Minas, } \\
\text { Minas Gerais }\end{array}$ & 486 & Students $6-8 y$ & $\mathrm{x}$ & No & Screen time & Typical day & $\begin{array}{l}\geq 85 \\
\text { percentile }\end{array}$ \\
\hline $\begin{array}{l}\text { Enes, Pegolo } \\
\text { and Silva } \\
(2009)^{203}\end{array}$ & Southeast & $\begin{array}{l}\text { Piedade, São } \\
\text { Paulo }\end{array}$ & 105 & Students $10-14$ y & $\mathrm{x}$ & No & Screen time & $\mathrm{x}$ & $>2 \mathrm{~h} /$ day \\
\hline $\begin{array}{l}\text { Enes and Slater } \\
(2013)^{204}\end{array}$ & Southeast & $\begin{array}{l}\text { Piracicaba, São } \\
\text { Paulo }\end{array}$ & 431 & Students $10-15 y$ & $\mathrm{x}$ & No & Screen time & $\begin{array}{l}\text { Weekdays and } \\
\text { weekend days }\end{array}$ & $\mathrm{x}$ \\
\hline $\begin{array}{l}\text { Romero et al. } \\
(2015)^{205}\end{array}$ & Southeast & $\begin{array}{l}\text { Piracicaba, São } \\
\text { Paulo }\end{array}$ & 454 & Students $10-14$ y & $\begin{array}{l}\text { Questionnaire } \\
\text { for Adolescents } \\
\text { Computerized } \\
\text { Version }\end{array}$ & Yes & Screen time & $\begin{array}{l}12 \text { months } \\
\text { prior }\end{array}$ & $\mathrm{x}$ \\
\hline $\begin{array}{l}\text { Werneck et al. } \\
(2018)^{206}\end{array}$ & Southeast & $\begin{array}{l}\text { Presidente } \\
\text { Prudente, São } \\
\text { Paulo }\end{array}$ & 280 & $\begin{array}{l}\text { Adolescents } 11 \\
-18 \mathrm{y}\end{array}$ & $\begin{array}{l}\text { Baecke } \\
\text { Questionnaire }\end{array}$ & No & $\begin{array}{l}\text { TV } \\
\text { watching }\end{array}$ & $\mathrm{x}$ & Very often \\
\hline $\begin{array}{l}\text { Tebar et al. } \\
(2018)^{207}\end{array}$ & Southeast & $\begin{array}{l}\text { Presidente } \\
\text { Prudente, São } \\
\text { Paulo }\end{array}$ & 1011 & Students $10-17 \mathrm{y}$ & $\mathrm{x}$ & No & Screen time & Week & $\begin{array}{l}\geq 22 \mathrm{~h} / \\
\text { week }\end{array}$ \\
\hline $\begin{array}{l}\text { Fernandes et al. } \\
(2008)^{208}\end{array}$ & Southeast & $\begin{array}{l}\text { Presidente } \\
\text { Prudente, São } \\
\text { Paulo }\end{array}$ & 1752 & Students & $\begin{array}{l}\text { Baecke } \\
\text { Questionnaire }\end{array}$ & No & $\begin{array}{l}\text { TV } \\
\text { watching }\end{array}$ & $\mathrm{x}$ & $\begin{array}{l}\text { High } \\
\text { frequency }\end{array}$ \\
\hline $\begin{array}{l}\text { Christofoletti et } \\
\text { al. }(2020)^{209}\end{array}$ & Southeast & $\begin{array}{l}\text { Rio Claro, São } \\
\text { Paulo }\end{array}$ & 482 & $\begin{array}{l}\text { Students elementary } \\
\text { education } 6 \text { th - 9th } \\
\text { grade and high } \\
\text { school 1st - 3rd } \\
\text { grade }\end{array}$ & $\begin{array}{l}\text { Sedentary } \\
\text { Behavior } \\
\text { Questionnaire }\end{array}$ & No & $\begin{array}{l}\text { Sitting time } \\
\text { and screen } \\
\text { time }\end{array}$ & $\mathrm{x}$ & $\geq 2 \mathrm{~h} /$ day \\
\hline $\begin{array}{l}\text { Straatmann et } \\
\text { al. }(2016)^{210}\end{array}$ & Southeast & $\begin{array}{l}\text { Rio de Janeiro } \\
\text { State }\end{array}$ & 526 & Students $10-19 y$ & $\mathrm{x}$ & No & Screen time & Typical day & $>4 \mathrm{~h} / \mathrm{day}$ \\
\hline $\begin{array}{l}\text { Tavares et al. } \\
(2014)^{211}\end{array}$ & Southeast & $\begin{array}{l}\text { Rio de Janeiro, } \\
\text { Rio de Janeiro }\end{array}$ & 174 & Students $13-17 y$ & $\begin{array}{l}\text { GSHS (version } \\
\text { used in PeNSE) }\end{array}$ & Yes & Screen time & $\begin{array}{l}\text { Typical } \\
\text { weekday }\end{array}$ & $\geq 2 \mathrm{~h} /$ day \\
\hline $\begin{array}{l}\text { Meziat Filho et } \\
\text { al. }(2015)^{212}\end{array}$ & Southeast & $\begin{array}{l}\text { Rio de Janeiro, } \\
\text { Rio de Janeiro }\end{array}$ & 1102 & $\begin{array}{l}\text { High school } \\
\text { students }\end{array}$ & $\begin{array}{l}\text { GSHS (version } \\
\text { used in PeNSE) }\end{array}$ & Yes & Screen time & Typical day & $\geq 2 \mathrm{~h} /$ day \\
\hline $\begin{array}{l}\text { Castro et al. } \\
(2008)^{213}\end{array}$ & Southeast & $\begin{array}{l}\text { Rio de Janeiro, } \\
\text { Rio de Janeiro }\end{array}$ & 1684 & Students 8th grade & $\mathrm{x}$ & No & Screen time & $\begin{array}{l}\text { Typical } \\
\text { weekday }\end{array}$ & $\geq 4 \mathrm{~h} /$ day \\
\hline $\begin{array}{l}\text { Lima et al. } \\
(2015)^{214}\end{array}$ & Southeast & $\begin{array}{l}\text { Santa Rita do } \\
\text { Sapucaí, Minas } \\
\text { Gerais }\end{array}$ & 175 & Students $6-17 y$ & $\mathrm{x}$ & No & $\begin{array}{l}\text { General } \\
\text { indicator } \\
\text { of SB }\end{array}$ & $\mathrm{x}$ & $\mathrm{x}$ \\
\hline $\begin{array}{l}\text { Melzer et al. } \\
(2015)^{215}\end{array}$ & Southeast & Santos, São Paulo & 357 & Children 3 - 10 y & $\begin{array}{l}\text { Youth } \\
\text { questionnaire of } \\
\text { the CELAFICS }\end{array}$ & No & Screen time & $\mathrm{x}$ & $>2 \mathrm{~h} /$ day \\
\hline $\begin{array}{l}\text { Nobre et al. } \\
(2016)^{216}\end{array}$ & Southeast & $\begin{array}{l}\text { São Paulo, São } \\
\text { Paulo }\end{array}$ & 255 & $\begin{array}{l}\text { Preschoolers } \\
3-5 y\end{array}$ & $\mathrm{x}$ & No & Screen time & $\mathrm{x}$ & $>2 \mathrm{~h} / \mathrm{day}$ \\
\hline $\begin{array}{l}\text { Leme and } \\
\text { Philippi } \\
(2015)^{217}\end{array}$ & Southeast & $\begin{array}{l}\text { São Paulo, São } \\
\text { Paulo }\end{array}$ & 253 & $\begin{array}{l}\text { Female } \\
\text { Adolescents }\end{array}$ & $\mathrm{x}$ & No & Screen time & $\begin{array}{l}\text { Weekdays and } \\
\text { weekend days }\end{array}$ & $>2 \mathrm{~h} /$ day \\
\hline $\begin{array}{l}\text { Lancarotte et al. } \\
(2010)^{218}\end{array}$ & Southeast & $\begin{array}{l}\text { São Paulo, São } \\
\text { Paulo }\end{array}$ & 2125 & $\begin{array}{l}\text { Students } 5 \text { th }-8 \text { th } \\
\text { grade }\end{array}$ & $\mathrm{x}$ & No & Screen time & $\mathrm{x}$ & $>2 \mathrm{~h} / \mathrm{day}$ \\
\hline
\end{tabular}

Continue... 
Continuation of Supplementary Table 3 - Main characteristics of studies that assessed sedentary behavior using subjective methods in children or adolescents.

\begin{tabular}{|c|c|c|c|c|c|c|c|c|c|}
\hline Author (year) & Region & $\begin{array}{l}\text { City-State or } \\
\text { State }\end{array}$ & $\mathrm{n}$ & Sample & $\begin{array}{l}\text { Questionnaire } \\
\text { used }\end{array}$ & $\begin{array}{l}\text { Quality } \\
\text { indicator? }\end{array}$ & SB indicator & $\begin{array}{l}\text { Time } \\
\text { reference }\end{array}$ & $\begin{array}{l}\text { Cut-off } \\
\text { point }\end{array}$ \\
\hline $\begin{array}{l}\text { Zapata et al. } \\
(2006)^{219}\end{array}$ & Southeast & $\begin{array}{l}\text { São Paulo, São } \\
\text { Paulo }\end{array}$ & 833 & Students & $\mathrm{x}$ & No & Screen time & $\begin{array}{l}\text { Weekdays and } \\
\text { weekend days }\end{array}$ & $\geq 2 \mathrm{~h} /$ day \\
\hline $\begin{array}{l}\text { Mazaro et al. } \\
(2011)^{220}\end{array}$ & Southeast & $\begin{array}{l}\text { Sorocaba, São } \\
\text { Paulo }\end{array}$ & 680 & Students $7-11 y$ & $\mathrm{x}$ & No & Screen time & $\mathrm{x}$ & $>2 \mathrm{~h} / \mathrm{day}$ \\
\hline $\begin{array}{l}\text { Silva et al. } \\
(2018)^{221}\end{array}$ & Southeast & $\begin{array}{l}\text { Uberaba, Minas } \\
\text { Gerais }\end{array}$ & 1125 & Students $5-18$ y & $\mathrm{x}$ & No & Screen time & $\mathrm{x}$ & $\geq 5 \mathrm{~h} / \mathrm{day}$ \\
\hline $\begin{array}{l}\text { Lourenço et al. } \\
(2017)^{222}\end{array}$ & Southeast & $\begin{array}{l}\text { Uberaba, Minas } \\
\text { Gerais }\end{array}$ & 1009 & Students $14-19 y$ & COMPAC & Yes & Screen time & $\mathrm{x}$ & $\geq 2 \mathrm{~h} /$ day \\
\hline $\begin{array}{l}\text { Andaki et al. } \\
(2018)^{223}\end{array}$ & Southeast & $\begin{array}{l}\text { Uberaba, Minas } \\
\text { Gerais }\end{array}$ & 1480 & Students $6-10 y$ & $\mathrm{x}$ & No & Screen time & $\begin{array}{l}\text { Weekdays and } \\
\text { weekend days }\end{array}$ & $\geq 2 \mathrm{~h} /$ day \\
\hline $\begin{array}{l}\text { Santos et al. } \\
(2013)^{224}\end{array}$ & Southeast & $\begin{array}{l}\text { Uberaba, Minas } \\
\text { Gerais }\end{array}$ & 649 & Students $9-12 y$ & $\begin{array}{l}\text { "Hábitos de Vida" } \\
\text { Questionnaire }\end{array}$ & No & Screen time & $\begin{array}{l}\text { Typical } \\
\text { weekday and } \\
\text { weekend day }\end{array}$ & $\geq 2 \mathrm{~h} /$ day \\
\hline $\begin{array}{l}\text { Andreoli et al. } \\
(2019)^{225}\end{array}$ & Southeast & $\begin{array}{l}\text { Viçosa, Minas } \\
\text { Gerais }\end{array}$ & 152 & Children 4- 7 y & $\mathrm{x}$ & No & $\begin{array}{l}\text { Sitting time } \\
\text { and screen } \\
\text { time }\end{array}$ & $\mathrm{x}$ & $\begin{array}{l}\text { screen } \\
\text { time: } \geq \\
3 \mathrm{~h} / \text { day; } \\
\text { sedentary } \\
\text { activities: } \geq \\
\text { 4h/day }\end{array}$ \\
\hline $\begin{array}{l}\text { Morais, } \\
\text { Miranda and } \\
\text { Priori }(2018)^{226}\end{array}$ & Southeast & $\begin{array}{l}\text { Viçosa, Minas } \\
\text { Gerais }\end{array}$ & 274 & $\begin{array}{l}\text { Female adolescent } \\
14-19 y\end{array}$ & $\begin{array}{l}\text { "Avaliação do } \\
\text { Tempo de Tela" } \\
\text { from Barros and } \\
\text { Navas }\end{array}$ & No & Screen time & Weekdays & $>2 \mathrm{~h} / \mathrm{day}$ \\
\hline $\begin{array}{l}\text { Prado Junior et } \\
\text { al. }(2015)^{227}\end{array}$ & Southeast & $\begin{array}{l}\text { Viçosa, Minas } \\
\text { Gerais }\end{array}$ & 676 & $\begin{array}{l}\text { Adolescents } 10 \\
-19 y\end{array}$ & $\mathrm{x}$ & No & Screen time & Typical week & $>2 \mathrm{~h} /$ day \\
\hline $\begin{array}{l}\text { Milagres et al. } \\
(2017)^{228}\end{array}$ & Southeast & $\begin{array}{l}\text { Viçosa, Minas } \\
\text { Gerais }\end{array}$ & 366 & Students $8-9 y$ & $\mathrm{x}$ & No & $\begin{array}{l}\text { Sitting time } \\
\text { and screen } \\
\text { time }\end{array}$ & $\mathrm{x}$ & $>2 \mathrm{~h} / \mathrm{day}$ \\
\hline $\begin{array}{l}\text { Molina et al. } \\
(2010)^{229}\end{array}$ & Southeast & Vitória-ES & 1282 & Students $7-10 y$ & $\mathrm{x}$ & No & Screen time & $\mathrm{x}$ & $\geq 4 \mathrm{~h} /$ day \\
\hline
\end{tabular}

Note: $\mathrm{y}$ = years; GSHS = Global School-based Student Health Survey; PeNSE = "Pesquisa Nacional de Saúde do Escolar"; PAQ-C = Physical Activity Questionnaire for Children; COMPAC = "Comportamentos dos adolescentes catarinenses"; YRBS = Youth Risk Behavior Survey; IPAQ = International Physical Activity Questionnaire; ASAQ = Adolescent sedentary activity questionnaire; Web-CAAFE = "Consumo Alimentar e Atividade Física de Escolares"; HELENA = Healthy Lifestyle in Europe by Nutrition in Adolescence; SB = sedentary behavior; TV = television; $\mathrm{h}=$ hours. 


\section{References of supplementary tables}

1. Ferrari GLM, Victo ER, Kovalskys I, Mello AV, Previdelli AN, Solé D et al. Sedentary behavior, physical activity and body composition in adults. Rev Assoc Med Bras. 2020;66(3):314-20.

2. Barbieri DF, Srinivasan D, Mathiassen SE, Oliveira AB. Comparison of Sedentary Behaviors in Office Workers Using Sit-Stand Tables With and Without Semiautomated Position Changes. Hum Factors. 2017;59(5):782-95.

3. Sasaki JE, Júnior JH, Meneguci J, Tribess $\mathrm{S}$, Marocolo Júnior M, Stabelini Neto A et al. Number of days required for reliably estimating physical activity and sedentary behaviour from accelerometer data in older adults. J Sports Sci. 2018;36(4):1572-7.

4. Mendonça G, Filho AP, Barbosa AO, Júnior JCF. Padrões de comportamento sedentário em adolescentes de um município da região Nordeste do Brasil. Rev Bras Ativ Fís Saúde. 2018;23:e0023.

5. Santos VOA, Browne RAV, Souza DC, Matos VAF, Macêdo GAD, Farias-Junior LF et al. Effects of High-Intensity Interval and Moderate-Intensity Continuous Exercise on Physical Activity and Sedentary Behavior Levels in Inactive Obese Males: A Crossover Trial. J Sports Sci Med. 2019;18(3):390-8.

6. Gerage $A M$, Benedetti $T R B$, Farah $B Q$, Santana FS, Ohara D, Andersen LB et al. Sedentary Behavior and Light Physical Activity Are Associated with Brachial and Central Blood Pressure in Hypertensive Patients. PloS One. 2015;10(12):e0146078.

7. Melo HN, Stoots SJ-M, Pool MA, Carvalho VO, Aragão MLDC, Gurgel RQ et al. Objectively measured physical activity levels and sedentary time in children and adolescents with sickle cell anemia. PloS One. 2018;13(12):e0208916.

8. Gonçalves PB, Hallal PC, Hino AAF, Reis RS. Individual and environmental correlates of objectively measured physical activity and sedentary time in adults from Curitiba, Brazil. Int J Public Health. 2017;62(7):831-40.

9. Bacil EDA, Watanabe PI, Silva MP, Fantinelli ER, Bozza R, Campos W. Validade de um questionário de comportamento sedentário em escolares de 9 a 15 anos de idade. $\mathrm{R}$ Bras $\mathrm{Ci}$ Saúde. 2018;22(4):341-8.

10. Santos CES, Manta SW, Maximiano GP, Confortin SC, Benedetti TRB, d'Orsi E et al. Accelerometer-Measured Physical Activity and Sedentary Behavior: A CrossSectional Study of Brazilian Older Adults. J Phys Act Health. 2018;15(11):811-8.

11. Costa BGG, Silva KS, George AM, Assis MAA. Sedentary behavior during school-time: Sociodemographic, weight status, physical education class, and school performance correlates in Brazilian schoolchildren. J Sci Med Sport. 2017;20(1):70-4.

12. Martins PC, Lima LRA, Silva AM, Petroski EL, Moreno YMF, Silva DAS. Phase angle is associated with the physical fitness of HIV-infected children and adolescents. Scand J Med Sci Sports. 2019;29(7):1006-12.

13. Furlanetto KC, Donária L, Schneider LP, Lopes JR, Ribeiro M, Fernandes KB et al. Sedentary Behavior Is an Independent Predictor of Mortality in Subjects With COPD. Respir Care. 2017;62(5):579-87.

14. Barbosa SC, Coledam DHC, Stabelini Neto A, Elias RGM, Oliveira AR. School environment, sedentary behavior and physical activity in preschool children. Rev Paul Pediatr. 2016;34(3):301-8.
15. Ramos DE, Bueno MRO, Vignadelli LZ, Werneck AO, Ronque ERV, Coelho-E-Silva MJ et al. Pattern of sedentary behavior in brazilian adolescents. Rev Bras Ativ Fís Saúde. 2018;23:e0009.

16. Silva J, Reichert F, Ohara D, Dourado A, Petroski E, Romanzini M. Validação dos limiares do acelerômetro Actical para a determinação do comportamento sedentário e da intensidade da atividade física em adolescentes. Rev Bras Ativ Fís Saúde. 2014;19(2):246-55.

17. Mielke GI, Brown WJ, Ekelund U, Brage S, Gonçalves $\mathrm{H}$, Wehrmeister FC et al. Socioeconomic position and sedentary behavior in Brazilian adolescents: A life-course approach. Prev Med. 2018;107(1):29-35.

18. Barbosa Porcellis da Silva R, Marques AC, Reichert FF. Objectively measured physical activity in Brazilians with visual impairment: description and associated factors. Disabil Rehabil. 2018;40(18):2131-7.

19. Horta BL, Schaan BD, Bielemann RM, Vianna CÁ, Gigante DP, Barros FC et al. Objectively measured physical activity and sedentary-time are associated with arterial stiffness in Brazilian young adults. Atherosclerosis. 2015;243(1):148-54.

20. Faria FR, Neves Miranda VP, Howe CA, Sasaki JE, Santos Amorim PR. Behavioral classes related to physical activity and sedentary behavior on the evaluation of health and mental outcomes among Brazilian adolescents. PloS One. 2020;15(6):e0234374.

21. Gomes EP, Reboredo MM, Carvalho EV, Teixeira DR, Carvalho LFC d'Ornellas, Filho GFF et al. Physical Activity in Hemodialysis Patients Measured by Triaxial Accelerometer. BioMed Res Int. 2015;2015:645645.

22. Lima-Junior D, Gantois P, Vasconcelos GC, Gonçalves R, Lamounier JA, Damasceno VO. Measurement of physical activity and sedentary behavior in adolescents by accelerometer: a cross-sectional study. Rev Bras Ativ Fís Saúde. 2019;24:e0104.

23. Paravidino VB, Mediano MFF, Sichieri R. Does a single bout of exercise influence subsequent physical activity and sedentary time in overweight boys? Physiol Behav. 2017;173:231-5.

24. Crisp AH, Verlengia R, Ravelli MN, Junior IR, Oliveira MRM. Changes in Physical Activities and Body Composition after Roux-Y Gastric Bypass Surgery. Obes Surg. 2018;28(6):1665-71.

25. Diniz TA, Rossi FE, Silveira LS, Neves LM, Fortaleza ACS, Christofaro DGD et al. The role of moderate-tovigorous physical activity in mediating the relationship between central adiposity and immunometabolic profile in postmenopausal women. Arch Endocrinol Metab. 2017;61(4):354-60.

26. Moura BP, Rufino RL, Faria RC, Sasaki JE, Amorim PRS. Can Replacing Sitting Time with Standing Time Improve Adolescents' Cardiometabolic Health? Int J Environ Res Public Health. 2019;16(17):3115.

27. Sperandio EF, Arantes RL, Chao TP, Romiti M, Gagliardi ART, Dourado VZ. Living near the port area is associated with physical inactivity and sedentary behavior. Sao Paulo Med J. 2017;135(1):34-41.

28. Ferrari GLM, Oliveira LC, Araujo TL, Matsudo V, Barreira TV, Tudor-Locke C et al. Moderate-to-Vigorous Physical Activity and Sedentary Behavior: Independent Associations With Body Composition Variables in Brazilian Children. Pediatr Exerc Sci. 2015;27(3):380-9. 
29. Lauria VT, Sperandio EF, Matheus AC, Silva RP, Romiti M, Gagliardi ART et al. High sedentary behavior and compromised physical capabilities in adult smokers despite the suitable level of physical activity in daily life. Rev Bras Cineantropom Desempenho Hum. 2017;19(1):62-73.

30. Gerage AM, Correia MA, Oliveira PML, Palmeira AC, Domingues WJR, Zeratti AE et al. Physical Activity Levels in Peripheral Artery Disease Patients. Arq Bras Cardiol. 2019;113(3):410-6.

31. Xavier RF, Pereira ACAC, Lopes AC, Cavalheri V, Pinto RMC, Cukier A et al. Identification of Phenotypes in People with COPD: Influence of Physical Activity, Sedentary Behaviour, Body Composition and Skeletal Muscle Strength. Lung. 2019;197(1):37-45.

32. Moreno NA, Aquino BG, Garcia IF, Tavares LS, Costa LF, Giacomassi IWS et al. Physiotherapist advice to older inpatients about the importance of staying physically active during hospitalisation reduces sedentary time, increases daily steps and preserves mobility: a randomised trial. J Physiother. 2019;65(4):208-14.

33. Caetano IT, Albuquerque MR, Nascimento FR, Mendes EL, Amorim PRS. Análise do comportamento sedentário de escolares por sexo, tipo de escola e turno escolar. Rev Bras Ci E Mov. 2015;24(1):16-26.

34. Fares D, Barbosa AR, Borgatto AF, Coqueiro RS, Fernandes $\mathrm{MH}$. Factors associated with nutritional status of the elderly in two regions of Brazil. Rev Assoc Medica Bras (English Edition). 2012;58(4):434-41.

35. Schuch FB, Bulzing RA, Meyer J, Vancampfort D, Firth J, Stubbs B et al. Associations of moderate to vigorous physical activity and sedentary behavior with depressive and anxiety symptoms in self-isolating people during the COVID-19 pandemic: A cross-sectional survey in Brazil. Psychiatry Res. 2020;292:113339.

36. Alves MS, Andrade RZ, Silva GC, Mota MC, Resende SG, Teixeira KR et al. Social Jetlag Among Night Workers is Negatively Associated with the Frequency of Moderate or Vigorous Physical Activity and with Energy Expenditure Related to Physical Activity.J Biol Rhythms. 2017;32(1):83-93.

37. Azevedo Barros MB, Lima MG, Medina LPB, Szwarcwald CL, Malta DC. Social inequalities in health behaviors among Brazilian adults: National Health Survey, 2013. Int J Equity Health. 2016;15(1):1-10.

38. Malta DC, Szwarcwald CL, Barros MBA, Gomes CS, Machado IE, Souza Júnior PRB et al. The COVID-19 Pandemic and changes in adult Brazilian lifestyles: a cross-sectional study, 2020. Epidemiol Serv Saúde. 2020;29(4):e2020407.

39. Vega JB, Poblacion AP, Taddei JAAC. Factors associated with the consumption of soft drinks among Brazilian preschool children: national survey of 2006. Cien Saude Colet. 2015;20(8):2371-80.

40. Mielke GI, Hallal PC, Malta DC, Lee I-M. Time trends of physical activity and television viewing time in Brazil: 20062012. Int J Behav Nutr Phys Act. 2014;11(1):1-9.

41. Garcia LMT, Silva KS, Del Duca GF, Costa FF, Nahas MV. Sedentary behaviors, leisure-time physical inactivity, and chronic diseases in Brazilian workers: a cross sectional study. J Phys Act Health. 2014;11(8):1622-34.

42. Cortes TR, Schlussel MM, Franco-Sena AB, Rebelo $\mathrm{F}, \mathrm{Kac} \mathrm{G}$. Television viewing and abdominal obesity in women according to smoking status: results from a large cross-sectional population-based study in Brazil. Rev Bras Epidemiol. 2013;16(1):137-45.
43. Knuth AG, Malta DC, Dumith SC, Pereira CA, Morais Neto OL, Temporão JG et al. Practice of physical activity and sedentarism among Brazilians: results of the National Household Sample Survey--2008. Cien Saude Colet. 2011;16(9):3697-705.

44. Epifânio SBO, Silveira JAC, Menezes RCE, Marinho PM, Brebal KMM, Longo-Silva G et al. Análise de série temporal do consumo de bebidas açucaradas entre adultos no Brasil: 2007 a 2014. Cien Saude Colet. 2020;25(7):2529-40.

45. Pitanga FJG, Matos SMA, Almeida MC, Griep RH, Viana MC, Melo ECP et al. Factors associated with sedentary behavior among ELSA-Brasil participants: ecological model. Rev Bras Ativ Fís Saúde. 2018;23:e0006.

46. Cássia Spanhol R, Bucalen-Ferrari CK. Obesity and lifestyle risk factors among an adult population in Legal Amazon, Mato Grosso, Brazil. Rev Salud Publica Bogota Colomb. 2016;18(1):26-36.

47. Santos RR, Rosa EC, Rosa T, Ferreira EA, Gris EF, Andrade RV et al. Sedentary Behavior: A Key Component in the Interaction between an Integrated Lifestyle Approach and Cardiac Autonomic Function in Active Young Men. Int J Environ Res Public Health. 2019;16:2156.

48. Mielke G, Costa D, Stopa S, Oliveira-Campos M, Pureza $\mathrm{D}$, Silva M. Tendência temporal de indicadores da prática de atividade física e comportamento sedentário nas capitais da Região Norte do Brasil: 2006-2013. Rev Bras Ativ Fís Saúde. 2015;20(2):130-40.

49. Moretti GS, Muniz PT, Tavares CM, Brunken GS, Farias Júnior JC, Farias ES et al. Prevalence of and factors associated with overweight among university students from Rio Branco, Acre - Brazil. Rev Bras Cineantropom Desempenho Hum. 2014;16(4):406-18.

50. Silva VD, Tribess S, Meneguci J, Sasaki JE, Santos DAT, Carneiro JAO et al. Time Spent in Sedentary Behaviour as Discriminant Criterion for Frailty in Older Adults. Int J Environ Res Public Health. 2018;15:1336.

51. Silva DAS, Mendonça BCA, Oliveira ACC. Qual é o impacto do comportamento sedentário na aptidão física de mulheres a partir de 50 anos de idade? Rev Bras Cineantropom Desempenho Hum. 2012;14(2):154-63.

52. Smith-Menezes A, Duarte MFS, Silva RJS. Inatividade física, comportamento sedentário e excesso de peso corporal associados à condição socioeconômica em jovens. Rev Bras Educ Fis. 2012;26(3):411-18.

53. Lourenço C, Sousa T, Fonseca S, Junior JV, Barbosa A. Comportamento sedentário em estudantes Universitários. Rev Bras Ativ Fís Saúde. 2016;21(1):67-77.

54. Goncalves WSF, Byrne R, Viana MT, Trost SG. Parental influences on screen time and weight status among preschool children from Brazil: a cross-sectional study. Int J Behav Nutr Phys Act. 2019;16(27):1-8.

55. Silva PAS, Rocha SV, Vasconcelos LRC, Santos CA, Silva PAS, Rocha SV et al. Comportamento sedentário como discriminador dos transtornos mentais comuns em idosos. J Bras Psiquiatr. 2017;66(4):183-8.

56. Toscano JJO, Zefferino ACG, Felix JBC, Cabral Júnior CR, Silva DAS. Pain prevalence on public servants: association with sedentary behavior and physical leisure activity. Rev Dor. 2016;17(2):106-10.

57. Tassitano R, Mielke G, Carvalho W, Oliveira M, Malta D. Tendência temporal de indicadores da prática de atividade física e comportamento sedentário nas capitais da Região Nordeste do Brasil: 2006-2013. Rev Bras Ativ Fís Saúde. 2015;20(2):152-64. 
58. Farah BQ, Barros MVG, Farias Júnior JC, Ritti-Dias RM, Lima RA, Barbosa JPAS et al. Percepção de estresse: associação com a prática de atividades físicas no lazer e comportamentos sedentários em trabalhadores da indústria. Rev Bras Educ Fis. 2013;27(2):225-34.

59. Mussi FC, Pitanga FJG, Pires CGS. Cumulative sitting time as discriminator of overweight, obesity, abdominal obesity and lipid disorders in nursing university. Rev Bras Cineantropom Desempenho Hum. 2017;19(1):40-9.

60. Marchesan M, Krug RR, Barbosa AR, Rombaldi AJ. Percepção de pacientes em hemodiálise sobre os benefícios e as modificações no comportamento sedentário após a participação em um programa de exercícios físicos. Rev Bras Ciênc Esporte. 2017;39(3):314-21.

61. Gonçalves PB, Lopes AAS, Silva AAP, Silva JSB, Silva AT, Becker LA et al. Combined physical activity and sitting time and their contribution to body mass index in adults. Rev Bras Cineantropom Desempenho Hum. 2017;19(2):174-84.

62. Felden ÉPG, Junior GJF, Andrade RD, Claumann GS, Pelegrini A, Teixeira CS. Fatores associados com a baixa duração do sono em universitários ingressantes. Rev Bras Ci E Mov. 2015;23(4):94-103.

63. Fronza FC, Berria J, Minatto G, Petroski EL, Fronza FC, Berria J et al. Exposure to simultaneous sedentary behavior domains and sociodemographic factorsassociated in public servants. Rev Bras Cineantropom Desempenho Hum. 2017;19(4):469-79.

64. Gutierres Filho PJB, Geraldo TDL, Bento GG, Silva FC, Arancibia BAV, Silva R. Qualidade de vida de idosos com deficiência e prática de atividade física em instituições de longa permanência. Rev Bras Geriatr E Gerontol. 2014;17(1):141-51.

65. Coledam DHC. Early and current physical activity: crosssectional associations with overweight among adults. J Prev Med Hyg. 2019;60(4):E354-60.

66. Souza SCS, Campanini MZ, Andrade SM, González AD, Melo JM, Mesas AE. Watching television for more than two hours increases the likelihood of reporting poor sleep quality among Brazilian schoolteachers. Physiol Behav. 2017;179:105-109.

67. Oliveira DV, Giacomin LL, Antunes MD, Granja CTL, Silva EC, Nascimento KVMO et al. Physical fitness of third-age-gym goers with systemic arterial hypertension in Maringá, Brazil. J Phys Educ. 2020;31:e3130.

68. Ribeiro AS, Pereira LC, Silva DRP, Santos LD, Schoenfeld BJ, Teixeira DC et al. Physical Activity and Sitting Time Are Specifically Associated With Multiple Chronic Diseases and Medicine Intake in Brazilian Older Adults. J Aging Phys Act. 2018;26(4):608-13.

69. Mielke GI, da Silva ICM, Owen N, Hallal PC. Brazilian adults' sedentary behaviors by life domain: population-based study. PloS One. 2014;9(3):e91614.

70. Bueno AF, Lemos FA, Ferrareze ME, Santos WAMD, Veronese FV, Dias AS. Muscle thickness of the pectoralis major and rectus abdominis and level of physical activity in chronic hemodialysis patients. Braz J Nephrol (J Bras Nefrol). 2017;39(4):391-7.

71. Leão OAA, Knuth AG, Meucci RD. Sedentary behavior in elderly residents from the rural area in Southern Brazil. Rev Bras Epidemiol. 2020;23:e200008.

72. Cafruni CB, Pattussi MP, Backes V, Costa JD, Olinto MTA, Bairros FS et al. Neighbourhood environment and transport-related and leisure-time sedentary behaviour amongst women in a city in Southern Brazil: a multilevel analysis. Int J Public Health. 2019;64(4):511-22.
73. Rech C, Reis R,Hino A, Sá GD, Stopa S,Malta D. Tendências temporais de indicadores de atividade física e comportamento sedentário nas capitais da Região Sul do Brasil: 2006-2013. Rev Bras Ativ Fís Saúde. 2015;20(1):47-56.

74. Leite JMRS, Soler JMP, Horimoto ARVR, Alvim RO, Pereira AC. Heritability and Sex-Specific Genetic Effects of Self-Reported Physical Activity in a Brazilian Highly Admixed Population. Hum Hered. 2019;84(3):151-8.

75. Genebra CVDS, Maciel NM, Bento TPF, Simeão SFAP, Vitta AD. Prevalence and factors associated with neck pain: a population-based study. Braz J Phys Ther. 2017;21(4):274-80.

76. Turi BC, Monteiro HL, Lemes ÍR, Codogno JS, Lynch KR, Asahi Mesquita CA et al. TV viewing time is associated with increased all-cause mortality in Brazilian adults independent of physical activity. Scand J Med Sci Sports. 2018;28(2):596-603.

77. Machado LAC, Viana JU, Silva SLA, Couto FGP, Mendes LP, Ferreira PH et al. Correlates of a Recent History of Disabling Low Back Pain in Community-dwelling Older Persons: The Pain in the Elderly (PAINEL) Study. Clin J Pain. 2018;34(6):515-24.

78. Moreira AD, Claro RM, Felisbino-Mendes MS, VelasquezMelendez G. Validity and reliability of a telephone survey of physical activity in Brazil. Rev Bras Epidemiol. 2017;20(1):136-46.

79. Duarte BM, Bernal RTI, Malta DC. Risk and protective factors for non communicable diseases in the Belo Horizonte population: Vigitel 2008. Rev Bras Epidemiol. 2013;16(3):572-81.

80. Carvalho ED, Valadares ALR, Costa-Paiva LH, Pedro AO, Morais SS, Pinto-Neto AM. Physical activity and quality of life in women aged 60 or older: associated factors. Rev Bras Ginecol Obstet. 2010;32(9):433-40.

81. Atalla M, Pinto AJ, Mielke GI, Benatti FB, Gualano B. Impact of a Real-World Lifestyle Intervention in an Entire Latin American City with More Than 50,000 People. Obes. 2019;27(12):1967-74.

82. Meneguci J, Sasaki JE, Silva Santos Á, Scatena LM, Damião R. Socio-demographic, clinical and health behavior correlates of sitting time in older adults. BMC Public Health. 2015;15(1):65.

83. Ribeiro FE, Vanderlei LCM, Palma MR, Tebar WR, Caldeira DT, Teles Fregonesi CEP et al. Body dissatisfaction and its relationship with overweight, sedentary behavior and physical activity in survivors of breast cancer. Eur J Obstet Gynecol Reprod Biol. 2018;229:153-8.

84. Oliveira CB, Franco MR, Maher CG, Tiedemann A, Silva FG, Damato TM et al. The efficacy of a multimodal physical activity intervention with supervised exercises, health coaching and an activity monitor on physical activity levels of patients with chronic, nonspecific low back pain (Physical Activity for Back Pain (PAyBACK) trial): study protocol for a randomised controlled trial. Trials. 2018;19:40.

85. Fernandes RA, Christofaro DGD, Casonato J, Costa Rosa CS, Costa FF, Freitas Júnior IF et al. Leisure time behaviors: prevalence, correlates and associations with overweight in Brazilian adults. A cross-sectional analysis. Rev Med Chil. 2010;138:29-35.

86. Fernandes RA, Mantovani AM, Codogno JS, Turi-Lynch BC, Pokhrel S, Anokye N et al. The Relationship between Lifestyle and Costs Related to Medicine Use in Adults. Arq Bras Cardiol. 2019;112(6):749-55.

87. Bertolini GN, Santos VR, Alves MJ, Cervellini PCM, Christofaro DGD, Santana LFD et al. Relation between high leisure-time sedentary behavior and low functionality in older adults. Rev Bras Cineantropom Desempenho Hum. 2016;18(6):713-21. 
88. Moraes SA, Checchio MV, Freitas ICM. Dyslipidemia and correlates in adults living in Ribeirão Preto, SP: results of the EPIDCV Project. Arq Bras Endocrinol Metabol. 2013;57(9):691-701.

89. Suzuki CS, Moraes SA, Freitas ICM. Sitting-time means and correlates in adults living in Ribeirão Preto-SP, Brazil, in 2006: OBEDIARP project. Rev Bras Epidemiol. 2010;13(4):699-712.

90. Gomes VB, Siqueira KS, Sichieri R. Physical activity in a probabilistic sample in the city of Rio de Janeiro. Cad Saúde Pública. 2001;17(4):969-76.

91. Monfort-Pires M, Salvador EP, Folchetti LD, SiqueiraCatania A, Barros CR, Ferreira SRG. Diet quality is associated with leisure-time physical activity in individuals at cardiometabolic risk. J Am Coll Nutr. 2014;33(4):297-305.

92. Monteiro CA, Florindo AA, Claro RM, Moura EC. Validade de indicadores de atividade física e sedentarismo obtidos por inquérito telefônico. Rev Saúde Pública. 2008;42(4):575-81.

93. Rocha BMC, Goldbaum M, César CLG, Stopa SR, Rocha BMC, Goldbaum $M$ et al. Comportamento sedentário na cidade de São Paulo: ISA-Capital 2015. Rev Bras Epidemiol. 2019;22: e190050.

94. Santos DAT, Virtuoso JS, Meneguci J, Sasaki JE, Tribess S. Combined Associations of Physical Activity and Sedentary Behavior With Depressive Symptoms in Older Adults. Issues Ment Health Nurs. 2017;38(3):272-6.

95. Silva LMA, Tavares DMS, Rodrigues LR. Transition and factors associated with the level of physical activity combined with sedentary behavior of the elderly: A longitudinal study. Biomédica. 2020;40(2):322-35.

96. Ferreira FG, Segheto W, Silva DCG, Pereira PF, Longo GZ. Visceral adiposity index associated with behavioral and inflammatory parameters in adults: a population based study. Nutr Hosp. 2018;35(2):326-31.

97. Martinho KO, Dantas EHM, Longo GZ, Ribeiro AQ, Pereira ET, Franco FS et al. Comparison of functional autonomy with associated sociodemographic factors, lifestyle, chronic diseases (CD) and neuropsychiatric factors in elderly patients with or without the metabolic syndrome (MS). Arch Gerontol Geriatr. 2013;57(2):151-5.

98. Costa CDS, Flores TR, Wendt A, Neves RG, Assunção MCF, Santos IS. Sedentary behavior and consumption of ultra-processed foods by Brazilian adolescents: Brazilian National School Health Survey (PeNSE), 2015. Cad Saúde Pública. 2018;34(3):e00021017.

99. Oliveira JS, Barufaldi LA, Abreu GA, Leal VS, Brunken GS, Vasconcelos SML et al. ERICA: use of screens and consumption of meals and snacks by Brazilian adolescents. Rev Saude Publica. 2016;50(Suppl 1):7s.

100. Rezende LFM, Azeredo CM, Canella DS, Claro RM, Castro IRR, Levy RB et al. Sociodemographic and behavioral factors associated with physical activity in Brazilian adolescents. BMC Public Health. 2014;14:485.

101. Hallal PC, Knuth AG, Cruz DKA, Mendes MI, Malta DC. Physical activity practice among Brazilian adolescents. Cien Saude Colet. 2010;15(Suppl.2):3035-42.

102. Nogueira JAD, Macedo da Costa TH. Gender differences in physical activity, sedentary behavior, and their relation to body composition in active Brazilian adolescents. J Phys Act Health. 2009;6(1):93-8.

103. Machado RHV, Iglesia I, Hartwick CA, Ursaia R, Fontanezi $\mathrm{N}$, Pires $\mathrm{A}$ et al. O que adolescentes brasileiros fisicamente ativos sabem sobre frutas e hortaliças? Conhecimento nutricional e fatores associados - Estudo Fitfood. J Hum Growth Dev. 2016;26(3):316-22.
104. Silva ACM, Cureau FV, Oliveira CL, Giannini DT, Bloch $\mathrm{KV}$, Kuschnir MCC et al. Physical activity but not sedentary time is associated with vitamin $\mathrm{D}$ status in adolescents: study of cardiovascular risk in adolescents (ERICA). Eur J Clin Nutr. 2019;73(3):432-40.

105. Hardman CM, Barros MVG, Lopes AS, Lima RA, Bezerra J, Nahas MV et al. Effectiveness of a schoolbased intervention regarding screen time in high school students. Rev Bras Cineantropom Desempenho Hum. 2014;16(Suppl.1):25-35.

106. Hackenhaar ML, Sichieri R, Muraro AP, Silva RMVG, Ferreira MG. Social mobility, lifestyle and body mass index in adolescents. Rev Saude Publica. 2013;47(5):942-51.

107. Alexandre M, Silva A, Coelho-Ravagnani C. Comportamento sedentário em adolescentes atendidos pela Estratégia de Saúde da Família em Cuiabá, Brasil. Rev Bras Ativ Fís Saúde. 2016;21(4):344-54.

108. Rauber SB, Castro HO, Marinho A, Vicente JB, Ribeiro HL, Monteiro LZ et al. Effects of a physical activity and nutritional intervention in overweight and obese children through an educational and recreational camp. Nutr Health. 2018;24(3):145-52.

109. Militão AG, Silva FR, Peçanha LM, Souza JWS, Militão ESG, Campbell CSG. Reprodutibilidade e validade de um questionário de avaliação do nível de atividade física e comportamento sedentário de escolares de 10 a 13 anos de idade, Distrito Federal, Brasil, 2012. Epidemiol E Serviços Saúde. 2013;22(11):111-20.

110. Santos MC, Castro Coutinho APC, Souza Dantas M, Yabunaka LAM, Guedes DP, Oesterreich SA. Correlates of metabolic syndrome among young Brazilian adolescents population. Nutr J. 2018;17:66.

111. Giugliano R, Carneiro EC. Factors associated with obesity in school children. J Pediatr (Rio J). 2004;80(1):17-22.

112. Gomes AC, Rebelo MAB, Queiroz AC, Queiroz Herkrath APC, Herkrath FJ, Rebelo Vieira JM et al. Socioeconomic status, social support, oral health beliefs, psychosocial factors, health behaviours and health-related quality of life in adolescents. Qual Life Res. 2020;29(1):141-51.

113. Pinheiro LE, Trindade RFC, Silva MAI, Machado DRL, Santos CB. Prática de atividade física de escolares do 4o e 5o anos do ensino fundamental da rede pública estadual. Rev Bras Med Esporte. 2017;23(4):308-13.

114. Bezerra ES, Neves EB, Rossato M, Santos JOL, Nahas MV, Barbosa RMSP. The influence of gender on the practice of physical activity in high school adolescents in the city of Manaus. Mundo Saúde Impr 2016;40(3):302-9.

115. Gordia AP, Quadros TMB, Silva LR, Mota J. Cut-off values for step count and TV viewing time as discriminators of hyperglycaemia in Brazilian children and adolescents. Ann Hum Biol. 2016;43(5):423-9.

116. Silva DAS, Santos Silva RJ. Association between sports participation and sedentary behavior during school recess among brazilian adolescents. J Hum Kinet 2015;45:225-32.

117. Silva DAS, Lima JO, Silva RJS, Prado RL. Nível de atividade física e comportamento sedentário em escolares. Rev Bras Cineantropom Desempenho Hum. 2009;11(3):299-306.

118. Pitanga FJ, Alves CF, Pamponet ML, Medina MG. Tempo de tela como discriminador de excesso de peso, obesidade e obesidade abdominal em adolescentes. Rev Bras Cineantropom Desempenho Hum. 2016;18(5):539-47.

119. Nunes MMA, Figueiroa JN, Alves JGB. Overweight, physical activity and foods habits in adolescents from different economic levels, Campina Grande (PB). Rev Assoc Med Bras. 2007;53(2):130-4. 
120. Costa IFAF, Medeiros CCM, Costa FDAF, Farias CRL, Souza DR, Adriano WS et al. Adolescentes: comportamento e risco cardiovascular. J Vasc Bras. 2017;16(3):205-13.

121. Aros LJL, Germano-Soares AH, Silva CRM, Silva AO, Tassitano RM, et al. Trends in television and computer/ videogame use and total screen time in high school students from Caruaru city, Pernambuco, Brazil: A repeated panel study between 2007 and 2012. Motriz Rev Educ Fís. 2017;23(Special Issue):e101793.

122. Petribú MMV, Tassitano RM, Nascimento WMF, Santos EMC, Cabral PC. Fatores associados ao sobrepeso e à obesidade em estudantes do ensino médio da rede pública estadual do município de Caruaru (PE). Rev Paul Pediatr. 2011;29(4):536-45.

123. Martins JS, Souza EA, Trompieri Filho N. Fatores associados ao tempo de tela em estudantes do ensino médio de Fortaleza, região Nordeste do Brasil. Sci Med. 2015;25(4):ID20985.

124. Bandeira AS, Silva KS, Sá SAM, Guerra PH, Mota J, Filho VCB. Effect of a multicomponent intervention on variables related to screen time in adolescents: a cluster-randomized controlled trial. Rev Bras Ativ Fís Saúde. 2018;23:e0005.

125. Munaro HLR, Silva DAS, Lopes ADS. Prevalence of excessive screen time and associated factors in a school from a city in the northeast of Brazil. J Hum Growth Dev. 2016;26(3):360-7.

126. Guedes DP, Souza MV, Ferreirinha JE, Silva AJRM. Physical activity and determinants of sedentary behavior in Brazilian adolescents from an underdeveloped region. Percept Mot Skills. 2012;114(2):542-52.

127. Farias Júnior JC, Lopes AS, Mota J, Hallal PC. Physical activity practice and associated factors in adolescents in Northeastern Brazil. Rev Saude Publica. 2012;46(3):505-15.

128. Silva KS, Lopes AS, Silva FM. Comportamentos sedentários associados ao excesso de peso corporal. Rev Bras Educ Fis. 2007;21(2):135-41.

129. Rivera IR, Silva MAM, Silva RDTA, Oliveira BAV, Carvalho ACC. Physical inactivity, TV-watching hours and body composition in children and adolescents. Arq Bras Cardiol. 2010;95(2):159-65.

130. Siqueira PP, Alves JGB, Figueiroa JN. Fatores associados ao excesso de peso em crianças de uma favela do Nordeste brasileiro. Rev Paul Pediatr. 2009;27(3):251-7.

131. Santos DRL, Lira PIC, Silva GAP, Santos DRL, Lira PIC, Silva GAP. Excess weight in preschool children: The role of food intake. Rev Nutr. 2017;30(1):45-56.

132. Oliveira LMFT, Ritti-Dias RM, Farah BQ, Christofaro DGD, Barros MVG, Diniz PRB et al. Does the type of sedentary behaviors influence blood pressurein adolescents boys and girls? A cross-sectional study. Cien Saude Colet. 2018;23(8):2575-85

133. Tassitano RM, Barros MVG,Tenório MCM,BezerraJ,Hallal PC. Prevalence of overweight and obesity and associated factors among public high school students in Pernambuco State, Brazil. Cad Saúde Pública. 2009;25(12):2639-52.

134. Lippo BRS, Silva IM, Aca CRP, Lira PIC, Silva GAP, Motta MEFA. Determinants of physical inactivity among urban adolescents. J Pediatr (Rio J). 2010;86(6):520-4.

135. Oliveira NKR, Lima RA, Mélo EN, Santos CM, Barros SSH, Barros MVG. Reprodutibilidade de questionário para medida da atividade física e comportamento sedentário em crianças pré-escolares. Rev Bras Ativ Fís Saúde. 2011;16(3):228-33.
136. Barbosa LMA, Arruda IKG, Canuto R, Lira PIC, Monteiro JS, Freitas DL et al. Prevalence and factors associated with excess weight in adolescents in a low-income neighborhood - Northeast, Brazil. Rev Bras Saúde Materno Infant .2019;19(3):661-70.

137. Brito Beck da Silva K, Ortelan N, Giardini Murta S, Sartori I, Couto RD, Leovigildo Fiaccone R et al. Evaluation of the Computer-Based Intervention Program Stayingfit Brazil to Promote Healthy Eating Habits: The Results from a School Cluster-Randomized Controlled Trial. Int J Environ Res Public Health. 2019;16:1674.

138. Alves CFA, Silva RCR, Assis AMO, Souza CO, Pinto EJ, Frainer DES. Factors associated with physical inactivity in adolescents aged 10-14 years, enrolled in the public school network of the city of Salvador, Brazil. Rev Bras Epidemiol. 2012;15(4):858-70.

139. Oliveira TC, Silva AAM, Santos CJN, Silva JS, Conceição SIO. Physical activity and sedentary lifestyle among children from private and public schools in Northern Brazil. Rev Saude Publica. 2010;44(6):996-1004.

140. Simões VMF, Batista RFL, Alves MTSSB, Ribeiro CCC, Thomaz EBAF, Carvalho CA et al. Saúde dos adolescentes da coorte de nascimentos de São Luís, Maranhão, Brasil, 1997/1998. Cad Saúde Pública. 2020;36(7):e00164519.

141. Silva RJS, Silva DAS, Oliveira AC. Low physical activity levels and associated factors in Brazilian adolescents from public high schools. J Phys Act Health. 2014;11(7):1438-45.

142. Menezes AS, Duarte MFS. Condições de vida, inatividade física e conduta sedentária de jovens nas áreas urbana e rural. Rev Bras Med Esporte. 2015;21(5):338-44.

143. Santos LS, Ribeiro DSS, Barreto ÍDC, Oliveira VHF, Silva DRP, Menezes AS. Association between insufficient level of physical activity and multiple health risk behaviors in adolescents. Motriz Rev Educ Fís. 2019;25(3): e101918.

144. Geremia R, Cimadon HMS, Souza WB, Pellanda LC. Childhood overweight and obesity in a region of Italian immigration in Southern Brazil: a cross-sectional study. Ital J Pediatr. 2015;41:28.

145. Suñé FR, Dias-da-Costa JS, Olinto MTA, Pattussi MP. Prevalence of overweight and obesity and associated factors among schoolchildren in a southern Brazilian city. Cad Saúde Pública. 2007;23(6):1361-71.

146. Vasques DG, Lopes AS. Fatores associados à atividade física e aos comportamentos sedentários em adolescentes. Rev Bras Cineantropom Desempenho Hum 2009;11(1):59-66.

147. Silva AF, Martins PC, Gonçalves ECA, Farias JM, Silva DAS, Silva AF et al. Prevalence and factors associated with sedentary behavior in the school recess among adolescents. Motriz Rev Educ Fís. 2018;24(4):e101834.

148. Azambuja APO, Oliveira ERN, Azambuja MA, Oliveira $\mathrm{AAB}$, Rinaldi W. Prevalência e fatores associados ao excesso de peso em escolares. Rev Baiana Saúde Pública. 2012;36(3):740-50.

149. Ulbricht L, Campos MF, Esmanhoto E, Ripka WL. Prevalence of excessive body fat among adolescents of a south Brazilian metropolitan region and State capital, associated risk factors, and consequences. BMC Public Health. 2018;18:312.

150. Prado CV, Rech CR, Hino AAF, Reis RS. Perception of neighborhood safety and screen time in adolescents from Curitiba, Brazil. Rev Bras Epidemiol. 2017;20(4):688-701.

151. Alberico CO,Schipperijn J, Reis RS. Use of global positioning system for physical activity research in youth: ESPAÇOS Adolescentes, Brazil. Prev Med. 2016;103(S):59-65. 
152. Machado-Rodrigues AM, Leite N, Coelho-e-Silva MJ, Enes F, Fernandes R, Mascarenhas LPG et al. Metabolic risk and television time in adolescent females. Int J Public Health. 2015;60(2):157-65.

153. Barbosa Filho VC, Campos W, Bozza R, Lopes AS. The prevalence and correlates of behavioral risk factors for cardiovascular health among Southern Brazil adolescents: a cross-sectional study. BMC Pediatr. 2012;12:130.

154. Guimarães RF, Silva MP, Mazzardo O, Martins RV, Campos W. Association between sedentary behavior and anthropometric and metabolic profiles among adolescents. Motriz Rev Educ Fís. 2013;19(4):753-62.

155. Silva MP, Fontana FE, Welk GJ, Saint-Maurice PF, Fantineli ER, Bacil EDA et al. Associations between physical activity, sedentary behavior, and health risk behaviors among adolescents from a city in Southern Brazil. Rev Bras Ativ Fís Saúde. 2019;24:e0068.

156. Schwertner DS, Oliveira RANS, Koerich MHAL, Motta AF, Pimenta AL, Gioda FR. Prevalence of low back pain in young Brazilians and associated factors: Sex, physical activity, sedentary behavior, sleep and body mass index. J Back Musculoskelet Rehabil. 2020;33(2):233-44.

157. Lobo AS, Assis MAA, Leal DB, Borgatto AF, Vieira FK, Di Pietro PF et al. Empirically derived dietary patterns through latent profile analysis among Brazilian children and adolescents from Southern Brazil, 2013-2015. PloS One. 2019;14:e0210425

158. Berria J, Minatto G, Lima LRA, Martins CR, Petroski EL. Predictors of dropout in the school-based multi-component intervention, 'Mexa-se'. Health Educ Res. 2018;33(4):280-91.

159. Pelegrini A, Petroski EL. Excesso de peso em adolescentes: prevalência e fatores associados. Rev Bras Ativ Fís Saúde. 2007;12(3):45-53.

160. Pinho MGM, Adami F, Benedet J, Vasconcelos FAG. Association between screen time and dietary patterns and overweight/obesity among adolescents. Rev Nutr. 2017;30(3):377-89.

161. Christofoletti M, Duca GFD, Gripa LT, Assis MAA. Comportamento sedentário no lazer e sua associação com atividade física no contexto escolar de crianças no sul do Brasil. J Phys Educ. 2016;27(1):e-2755.

162. Costa FF, Assis MAA. Nível de atividade física e comportamentos sedentários de escolares de sete a dez anos de Florianópolis-SC. Rev Bras Ativ Fís Saúde. 2011;16(1):48-54.

163. Oliveira RG, Guedes DP. Determinants of lifestyle associated with metabolic syndrome in Brazilian adolescents. Nutr Hosp. 2019;36(4):826-33.

164. Silva MP, Gasparotto GS, Smolarek AC, Dellagrana RA, Mascarenhas LPG, Campos W et al. Comportamento sedentário relacionado ao sobrepeso e obesidade em crianças e adolescentes. Pensar a Prát. 2010;13(2):1-15.

165. Werneck AO, Silva DR, Agostinete RR, Fernandes RA, Ronque ERV, Cyrino ES. Social, behavioral and biological correlates of cardiorespiratory fitness according to sex, nutritional status and maturity status among adolescents. A cross-sectional study. Sao Paulo Med J. 2018;136(3):237-44.

166. Greca JPA, Silva DAS, Loch MR. Physical activity and screen time in children and adolescents in a medium size town in the South of Brazil. Rev Paul Pediatr. 2016;34(3):316-22.

167. Silva DR, Ohara D, Tomeleri CM, Batista MB, Fernandes RA, Ronque ER et al. Association between risk behaviors and adiposity indicators in adolescents from Southern Brazil: A methodological approach. J Child Health Care. 2016;20(3):314-23.
168. Christofaro DGD, Andrade SM, Cardoso JR, Mesas AE, Codogno JS, Fernandes RA. High blood pressure and sedentary behavior in adolescents are associated even after controlling for confounding factors. Blood Press. 2015;24(5):317-23.

169. Coledam DHC, Ferraiol PF, Pires R, Ribeiro EAG, Ferreira MAC, Oliveira AR. Agreement between two cutoff points for physical activity and associated factors in young individuals. Rev Paul Pediatr. 2014;32(3):215-22.

170. Felden ÉPG, Filipin D, Barbosa DG, Andrade RD, Meyer C, Louzada FM. Factors associated with short sleep duration in adolescents. Rev Paul Pediatr. 2016;34(1):64-70.

171. Moraes ACF, Fernandes CAM, Elias RGM, Nakashima ATA, Reichert FF, Falcão MC. Prevalence of physical inactivity and associated factors in adolescents. Rev Assoc Médica Bras. 2009;55(5):523-8.

172. Guimarães RF, Silva MP, Legnani E, Mazzardo O, Campos W. Reprodutibilidade de questionário de atividades sedentárias para adolescentes brasileiros. Rev Bras Cineantropom Desempenho Hum. 2013;15(3):276-85.

173. Oliveira A, Guilherme FR, Santos SR, Fávero MTM, Guilherme VR, Rinaldi W et al. Prediction of cardiorrespiratory fitness by screen time in schoolchildrens. J Phys Educ. 2020;31:e3139.

174. Ferreira RW, Rombaldi AJ, Ricardo LIC, Hallal PC, Azevedo MR. Prevalence of sedentary behavior and its correlates among primary and secondary school students. Rev Paul Pediatr. 2016;34(1):56-63.

175. Hallal PC, Bertoldi AD, Gonçalves H, Victora CG. Prevalence of sedentary lifestyle and associated factors in adolescents 10 to 12 years of age. Cad Saúde Pública. 2006;22(6):1277-87.

176. Xavier M, Bielemann R, Maciel F, Neutzling M, Gigante D. Variação temporal no excesso de peso e obesidade em adolescentes de escola privada do Sul do Brasil. Rev Bras Ativ Fís Saúde. 2014;19(1):74-85.

177. Bacil E, Rech C, Hino A. Padrões de atividade física em escolares de Ponta Grossa, Paraná. Rev Bras Ativ Fís Saúde. 2013;18(2):177-85.

178. Lopes AS, Silva KS, Barbosa Filho VC, Bezerra J, Oliveira ESA, Nahas MV. Trends in screen time on week and weekend days in a representative sample of Southern Brazil students. J Public Health. 2014;36(4):608-14.

179. Corso ACT, Caldeira GV, Fiates GMR, Schmitz BAS, Ricardo GD, Vasconcelos FAG. Fatores comportamentais associados ao sobrepeso e à obesidade em escolares do Estado de Santa Catarina. R Bras Est Pop. 2012;29(1):117-31.

180. Silva KS, Nahas MV, Hoefelmann LP, Lopes AS, Oliveira ES. Associações entre atividade física, índice de massa corporal e comportamentos sedentários em adolescentes. Rev Bras Epidemiol. 2008;11(1):159-68.

181. Spohr CF, Walker D, Azambuja CR, Farinha JB, Azevedo MR, Santos DL. Nível de atividade física de crianças do 10 ano do ensino fundamental. $\mathrm{R}$ Bras $\mathrm{Ci} \mathrm{E}$ Mov. 2012;20(4):106-11.

182. Faria WF, Farias JP, Ronque ERV, Santos CF, Stabelini Neto A, Elias RGM. Atividade física, comportamento sedentário e aptidão física de escolares: comparação entre período integral e parcial. Rev Bras Cineantropom Desempenho Hum. 2015;17(4):418-27.

183. Piola TS, Bacil EDA, Silva MP, Pacífico AB, Camargo EM, Campos $\mathrm{W}$ et al. Impact of physical activity correlates in the isolated and combined presence of insufficient level of physical activity and high screen time among adolescents. Rev Paul Pediatr. 2019;37(2):194-201. 
184. Gonçalves ECA, Silva DAS. Factors associated with low levels of aerobic fitness among adolescents. Rev Paul Pediatr. 2016;34(2):141-7.

185. Weber AP, Henn RL, Vicenzi K, Backes V, Paniz VMV, Olinto MTA. Adesão aos '10 passos da alimentação saudável para crianças' e fatores associados em escolares. Rev Nutr. 2015;28(3):289-304.

186. Beck CC, Lopes AS, Farias Júnior JC, Beck CC, Lopes AS, Farias Júnior JC. Factors associated with serum lipids of adolescents from the Brazilian South. Rev Nutr. 2014;27(1):35-43.

187. Silva T, Oliveira H, Bergmann M, Bergmann G. Associação entre atividade física e tempo de tela com o nível socioeconômico em adolescentes. Rev Bras Ativ Fís Saúde. 2015;20(5):503-13.

188. Bordon S, Srebernich SM, Bernardi JLD, Merhi VAL, Bordon S, Srebernich SM et al. Screen time, body mass index and neck circumference: is there an association with social class in children? Rev Bras Cineantropom Desempenho Hum. 2019;21:e58235.

189. Araújo LGM, Turi BC, Locci B, Mesquita CAA, Fonsati NB, Monteiro HL. Patterns of Physical Activity and Screen Time Among Brazilian Children. J Phys Act Health. 2018;15(6):457-61.

190. Vitta A, Martinez MG, Piza NT, Simeão SFDAP, Ferreira NP. Prevalence of lower back pain and associated factors in students. Cad Saúde Pública. 2011;27(8):1520-8.

191. Vitta AD, Barros DS, Palma R, Soares MVF, Conti MHSD, Gatti MAN et al. Prevalência e fatores associados à adiposidade central e periférica em estudantes do ensino fundamental brasileiro. J Hum Growth Dev. 2013;23(3):365-71.

192. Mondini L, Levy RB, Saldiva SRDM, Venâncio SI, Azevedo Aguiar J, Stefanini MLR. Overweight, obesity and associated factors in first grade schoolchildren in a city of the metropolitan region of São Paulo, Brazil. Cad Saúde Pública. 2007;23(8):1825-34.

193. Braz M, Assumpção D, Barros MBA, Barros Filho AA. Added sugar intake by adolescents: A population-based study. Cien Saude Colet. 2019; 24(9):3237-46.

194. Andrade Neto F, Eto FN, Pereira TSS, Carletti L, Molina MCB. Active and sedentary behaviours in children aged 7 to 10 years old: the urban and rural contexts, Brazil. BMC Public Health. 2014;14:1174.

195. Souza VZ, Reneis MDM, Dalbello MO, Gonçalves LM, Rezende TM, Silva Júnior AJ. Correlação entre atividade física, repouso, riscos cardiovasculares e obesidade em crianças. R Bras Ci Saúde. 2016;20(3):107-14.

196. Victo ER, Ferrari GLM, Silva Junior JP, Araújo TL, Matsudo VKR. Lifestyle indicators and cardiorespiratory fitness in adolescents. Rev Paul Pediatr. 2017;35(1):61-8.

197. Vasconcellos MB, Anjos LA, Vasconcellos MTL. Nutritional status and screen time among public school students in Niterói, Rio de Janeiro State, Brazil. Cad Saúde Pública. 2013;29(4):713-22.

198. Silva RC, Malina RM. Level of physical activity in adolescents from Niterói, Rio de Janeiro, Brazil. Cad Saúde Pública. 2000;16(4):1091-7.

199. Fonseca VM, Sichieri R, Veiga GV. Factors associated with obesity among adolescents. Rev Saúde Pública. 1998;32(6):541-9.
200. Rodrigues RRM, Souza BSN, Cunha DB, Estima CCP, Sichieri R, Yokoo EM. Association between screen time and the variation of food intake markers among schoolaged adolescents in Niterói/RJ, Brazil. Cad Saúde Colet. 2020;28(1):24-33.

201. Fernandes JAA, Genebra CVS, Maciel NM, Fiorelli A, Conti MHS, Vitta AD. Dor lombar em escolares: um estudo transversal em uma cidade do oeste de São Paulo, Brasil. Acta Ortop Bras. 2015;23(5):235-8

202. Caixeta HCV, Amato AA. Factors associated with overweight and abdominal obesity in Brazilian schoolaged children: a comprehensive approach. Arch Endocrinol Metab. 2020;64(4):445-53.

203. Enes CC, Pegolo GE, Silva MV. Influência do consumo alimentar e do padrão de atividade física sobre o estado nutricional de adolescentes de Piedade, São Paulo. Rev Paul Pediatr. 2009;27(3):265-71.

204. Enes CC, Slater B. Variation in dietary intake and physical activity pattern as predictors of change in body mass index (BMI) Z-score among Brazilian adolescents. Rev Bras Epidemiol. 2013;16(2):493-501.

205. Romero A, Borges C, Villar B. Patterns of physical activity and sedentary behavior associated with overweight in brazilian adolescents. Rev Bras Ativ Fís Saúde. 2015;20(1):26-35.

206. Werneck AO, Agostinete RR, Cayres SU, Urban JB, Wigna A, Chagas LGM et al. Association between Cluster of Lifestyle Behaviors and HOMA-IR among Adolescents: ABCD Growth Study. Medicina (Kaunas). 2018;54(6):96.

207. Tebar WR, Ritti-Dias RM, Farah BQ, Zanuto EF, Vanderlei LCM, Christofaro DGD. High blood pressure and its relationship to adiposity in a school-aged population: body mass index vs waist circumference. Hypertens Res. 2018;41(2):135-40.

208. Fernandes RA, Júnior IFF, Cardoso JR, Vaz Ronque ER, Loch MR, Oliveira AR. Association between regular participation in sports and leisure time behaviors in Brazilian adolescents: a cross-sectional study. BMC Public Health. 2008;8:329.

209. Christofoletti AEM, Benites LC, Sebastião E, Ueno DT, Nakamura PM. Prevalence and sociodemographic factors associated with sedentary behavior in adolescents from Rio Claro-SP, Brazil. J Phys Educ. 2020;31:e3159.

210. Straatmann VS, Oliveira AJ, Rostila M, Lopes CS. Changes in physical activity and screen time related to psychological well-being in early adolescence: findings from longitudinal study ELANA. BMC Public Health. 2016;16:977.

211. Tavares LF, Castro IRR, Cardoso LO, Levy RB, Claro RM, Oliveira AF. Validity of indicators on physical activity and sedentary behavior from the Brazilian National SchoolBased Health Survey among adolescents in Rio de Janeiro, Brazil. Cad Saúde Pública. 2014;30(9):1861-74.

212. Meziat Filho N, Coutinho ES, Azevedo e Silva G. Association between home posture habits and low back pain in high school adolescents. Eur Spine J. 2015;24(3):425-33.

213. Castro IRR, Cardoso LO, Engstrom EM, Levy RB, Monteiro CA. Surveillance of risk factors for non-communicable diseases among adolescents: the experience in Rio de Janeiro, Brazil. Cad Saúde Pública. 2008; 24(10):2279-88.

214. Lima MCC, Romaldini CC, Romaldini JH. Frequency of obesity and related risk factors among school children and adolescents in a low-income community. A cross-sectional study. Sao Paulo Med J. 2015;133(2):125-30.

215. Melzer MRTF, Magrini IM, Domene SMÁ, Martins PA. Factors associated with abdominal obesity in children. Rev Paul Pediatr. 2015;33(4):437-44. 
216. Nobre ÉB, Brentani AVM, Ferraro AA. Association between maternal lifestyle and preschool nutrition. Rev Assoc Med Bras. 2016;62(6):494-505.

217. Leme ACB, Philippi ST. The 'Healthy Habits, Healthy Girls' randomized controlled trial for girls: study design, protocol, and baseline results. Cad Saúde Pública. 2015;31(7):1381-94.

218. Lancarotte I, Nobre MR, Zanetta R, Polydoro M. Lifestyle and cardiovascular health in school adolescents from São Paulo. Arq Bras Cardiol. 2010;95:61-9.

219.Zapata AL, Moraes AJP, Leone C, Doria-Filho U, Silva CAA. Pain and musculoskeletal pain syndromes related to computer and video game use in adolescents. Eur J Pediatr. 2006;165(6):408-14.

220. Mazaro IAR, Zanolli ML, Antonio MÂRGM, Morcillo AM, Zambon MP. Obesity and cardiovascular risk factors in school children from Sorocaba, SP. Rev Assoc Med Bras. 2011;57(6):660-6.

221. Silva AP, Feilbelmann TCM, Silva DC, Palhares HMC, Scatena LM, Resende EAMR et al. Prevalence of overweight and obesity and associated factors in school children and adolescents in a medium-sized Brazilian city. Clinics. 2018;73:e438.

222. Lourenço CLM, Pires MR, Leite MAFJ, Sousa TF, Mendes EL. Deslocamento passivo para escola e fatores associados em adolescentes. J Phys Educ. 2017;28:e2831.

223. Andaki ACR, Mendes EL, Brito CJ, Amorim PRS, Wood $\mathrm{R}$, Tinoco ALA et al. Prevalence and factors associated with metabolic syndrome in 6-10-year-old children. Motriz Rev Educ Fís. 2018;24(3):e0062-18.

224. Santos A, Andaki ACR, Amorim PRS, Mendes EL. Fatores associados ao comportamento sedentário em escolares de 9-12 anos de idade. Motriz Rev Educ Fís. 2013;19(3):S25-S34.

225. Andreoli CS, Vieira-Ribeiro SA, Fonseca PCA, Moreira AVB, Ribeiro SMR, Morais MB et al. Eating habits, lifestyle and intestinal constipation in children aged four to seven years. Nutr Hosp. 2019;36(1):25-31.

226. Morais NS, Miranda VPN, Priore SE. Body image of female adolescents and its association with body composition and sedentary behavior. Cien Saude Colet. 2018;23(8):2693-703.

227. Prado Junior PP, Faria FR, Faria ER, Franceschini SCC, Priore SE. Cardiovascular risk and associated factors in adolescents. Nutr Hosp. 2015;32(2):897-904.

228. Milagres LC, Rocha NP, Albuquerque FM, Castro APP, Filgueiras MS, Pessoa MC et al. Sedentary behavior is associated with lower serum concentrations of vitamin D in Brazilian children. Public Health. 2017;152:75-78.

229. Molina MCB, Faria CP, Montero MP, Cade NV, Mill JG. Cardiovascular risk factors in 7-to-10-year-old children in Vitória, Espírito Santo State, Brazil. Cad Saúde Pública. 2010;26(5):909-17. 